\title{
Dynamic Kinetic Resolution of Azlactones Catalyzed by Chiral Brønsted Acids
}

\author{
Guojian Lu and Vladimir B. Birman*
}

A contribution from the Department of Chemistry, Washington University, Campus Box 1134, One Brookings Drive, Saint Louis, Missouri 63130

SUPPORTING INFORMATION: Experimental procedures (11 pages), ${ }^{1} \mathrm{H}$ NMR and ${ }^{13} \mathrm{C}$ NMR spectra (52 pages).

1. General. All reagents were obtained commercially and used as received unless otherwise specified. The BINOL phosphoric acid catalysts 3a-g were prepared according to a combination of published procedures. ${ }^{1}$ Synthesis and characterization of the previously unreported phosphoric acid $3 \mathbf{h}$ is described below. Azlactone substrates were prepared by dehydration of the corresponding ( \pm - $\mathrm{N}$-aroyl- $\alpha$-amino acids with $\mathrm{N}, \mathrm{N}$-dicyclohexylcarbodiimide ${ }^{2}$ and were recrystallized prior to the reaction. Commercial samples of 1-naphthylmethanol were typically contaminated with up to $5 \%$ of the 2-naphthyl isomer, which was removed by recrystallization from hexanes.

Solvents used for chromatography were ACS or HPLC grade, as appropriate. Reactions were monitored by thin layer chromatography (TLC) and by 1H NMR. EM Science 60F silica gel plates were used for TLC analyses. Flash column chromatography was performed over ICN Ecochrom silica gel $(32-63 \mathrm{~mm})$. HPLC analyses were performed on a Shimadzu LC system using Chiralcel OD-H, Chiralpak AD and Chiralpak AD-H analytical chiral stationary phase columns (4.6x250 mm, Chiral Technologies, Inc.). ${ }^{1} \mathrm{H}$ NMR and ${ }^{13} \mathrm{C}$ NMR spectra were recorded on a Mercury $300 \mathrm{MHz}$ Varian spectrometer. The chemical shifts are reported as d values (ppm) relative to TMS using residual $\mathrm{CHCl}_{3}$ peak $(7.26 \mathrm{ppm})$ as the reference. High Resolution mass spectral analyses were performed at Washington University MS Center on a Kratos MS-50TA spectrometer using Electro Spray Ionization (ESI) method. Melting points were measured on a Mel-Temp II capillary melting point apparatus. Infrared spectra were recorded on a Perkin-Elmer Spectrum Bx FTIR spectrophotometer using potassium bromide plates.

\section{Synthesis of catalyst $3 h$}

2a. (R)-3,3'-bis-(9-phenylanthracen-10-yl)-2,2'-dihydroxy-1,1'-binapthyl.

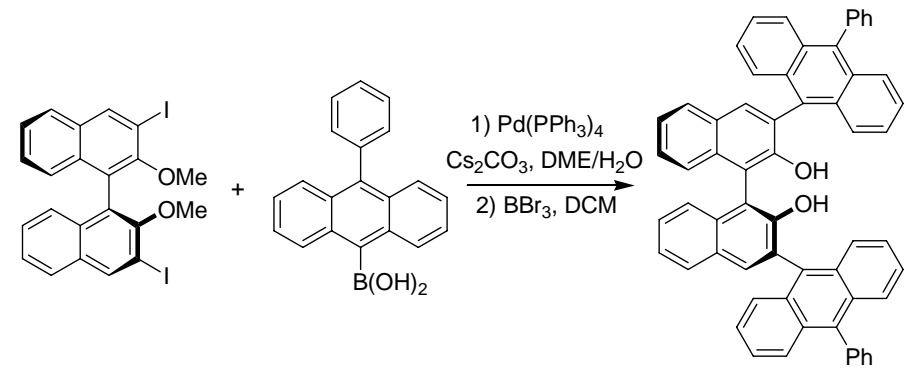

To a mixture of (R)-3,3'-diiodo-2,2'-dimethoxy-1,1'-binapthyl ${ }^{[1 \mathrm{a}]}$ (297 mg, $\left.0.53 \mathrm{mmol}\right)$, 9phenylanthracen-10-ylboronic acid ${ }^{[3]}(650 \mathrm{mg}, 2.18 \mathrm{mmol}), \mathrm{Pd}\left(\mathrm{PPh}_{3}\right)_{4}(66 \mathrm{mg}, 0.053 \mathrm{mmol})$ and $\mathrm{Cs}_{2} \mathrm{CO}_{3}(1.04 \mathrm{~g}, 3.18 \mathrm{mmol})$ was added $32 \mathrm{~mL}$ of degassed aqueous 1,2-dimethoxyethane 
$\left(\mathrm{DME} / \mathrm{H}_{2} \mathrm{O}=3 / 1\right)$ under argon. Then the mixture was stirred under reflux for $24 \mathrm{~h}$, cooled to room temperature, extracted with $\mathrm{CH}_{2} \mathrm{Cl}_{2}$, filtered through Celite, washed with saturated aqueous $\mathrm{NH}_{4} \mathrm{Cl}$ solution, water, and brine, dried with $\mathrm{Na}_{2} \mathrm{SO}_{4}$ and concentrated on a rotary evaporator. The resulting crude intermediate was dissolved in $20 \mathrm{~mL}$ of $\mathrm{CH}_{2} \mathrm{Cl}_{2}$ and treated at $0{ }^{\circ} \mathrm{C}$ with $\mathrm{BBr}_{3}$ $(0.35 \mathrm{~mL}, 3.71 \mathrm{mmol})$. The reaction mixture was allowed to warm to room temperature and stirred overnight. It was then quenched with water, washed with saturated aqueous $\mathrm{NaHCO}_{3}$, water, and brine, dried by $\mathrm{Na}_{2} \mathrm{SO}_{4}$, and rotary evaporated. Column chromatography (Hexane/EtOAc $=20: 1)$ gave 0.35 g of beige powder $\left(83 \%\right.$ yield). ${ }^{1} \mathbf{H N M R}\left(300 \mathrm{MHz}, \mathrm{CDCl}_{3}\right)$ : $\delta 8.10(\mathrm{~s}, 2 \mathrm{H}), 7.98-7.92(\mathrm{~m}, 4 \mathrm{H}), 7.78-7.71(\mathrm{~m}, 6 \mathrm{H}), 7.65-7.37(\mathrm{~m}, 20 \mathrm{H}), 7.34-7.23(\mathrm{~m}, 4 \mathrm{H})$; ${ }^{13}$ C NMR $\left(75 \mathrm{MHz}, \mathrm{CDCl}_{3}\right): \delta 151.34,139.07,138.59,134.21,133.47,131.48,131.06,130.77$, $130.72,130.36,130.31,129.62,128.76,128.67,127.82,127.72,127.68,127.63,127.52,126.50$, 126.43, 126.12, 125.49, 125.19, 124.56, 113.77; IR (KBr, cm $\left.{ }^{-1}\right)$ 3529, 3059, 1497, 1438, 1379, 1259, 1208, 1091, 1025, 912, 770, 747, 702,611. MS: HR-ESI calculated for $\mathrm{C}_{60} \mathrm{H}_{38} \mathrm{O}_{2}[\mathrm{M}+\mathrm{Na}]$ : 813.2764, found: 813.2763. $[\alpha]_{\mathbf{D}}=+69.7^{\circ}\left(\right.$ c $\left.0.4, \mathrm{CH}_{2} \mathrm{Cl}_{2}\right)$

\section{2b. (R)-3,3'-bis-(9-phenylanthracen-10-yl)-1,1'-binapthyl-2,2'-diyl-phosphoric acid.}
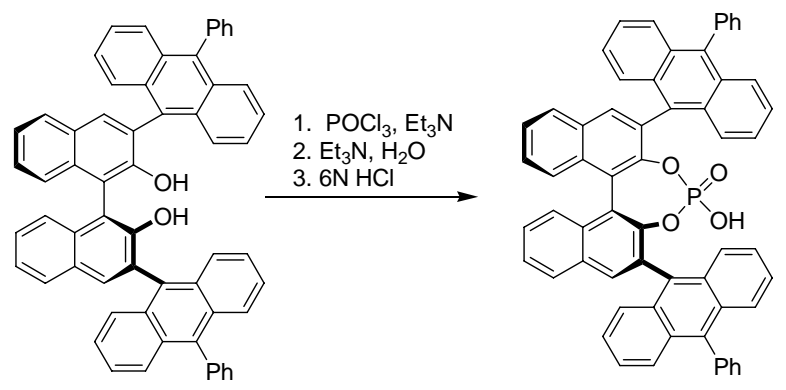

To a solution of the BINOL derivative prepared above $(100 \mathrm{mg}, 0.126 \mathrm{mmol})$ in $15 \mathrm{~mL}$ of $\mathrm{CH}_{2} \mathrm{Cl}_{2}$ stirring at $0{ }^{\circ} \mathrm{C}$ was added $\mathrm{Et}_{3} \mathrm{~N}(88 \mu \mathrm{l}, 0.630 \mathrm{mmol})$, followed by $\mathrm{POCl}_{3}(18 \mu \mathrm{l}, 0.190$ $\mathrm{mmol}$ ), and the mixture was allowed to warm to room temperature and stirred overnight, whereupon the reaction was complete by TLC. The mixture was treated with $2 \mathrm{~mL}$ of $\mathrm{Et}_{3} \mathrm{~N}$ and $10 \mathrm{~mL}$ of $\mathrm{H}_{2} \mathrm{O}$ stirred for one more day and acidified with $6 \mathrm{~N} \mathrm{HCl}$ to adjust the $\mathrm{pH}$ to 2 . After stirring for $2 \mathrm{~h}$, the mixture was washed with $\mathrm{H}_{2} \mathrm{O}$ and brine, dried by $\mathrm{Na}_{2} \mathrm{SO}_{4}$, and rotary evaporated. Column chromatography $\left(\mathrm{CH}_{2} \mathrm{Cl}_{2} / \mathrm{MeOH}=100: 1\right.$ to $\left.100: 2\right)$ yielded $85 \mathrm{mg}$ of the product as pale orange powder (79\% yield). ${ }^{1}$ HNMR $\left(300 \mathrm{MHz}, \mathrm{CDCl}_{3}\right): \delta 7.97(\mathrm{~s}, 2 \mathrm{H}), 7.90(\mathrm{~d}$, $J=7.8 \mathrm{~Hz}, 2 \mathrm{H}), 7.82(\mathrm{~d}, J=8.4 \mathrm{~Hz}, 2 \mathrm{H}), 7.71(\mathrm{~d}, J=8.1 \mathrm{~Hz}, 2 \mathrm{H}), 7.56-7.18(\mathrm{~m}, 20 \mathrm{H}), 7.07-7.05$ (m, 4H), 6.75-6.70 (m, 2H), 6.47-6.44 (m, 2H); ${ }^{13}$ C NMR (75 MHz, $\left.\mathrm{CDCl}_{3}\right): \delta 148.19,138.98$, $137.60,133.51,133.24,132.83$, 131.56, 131.40, 130.53, 130.23, 129.68, 129.61, 128.75, 128.58, $127.80,127.43,127.17,127.06,126.51,126.24,126.14,125.90,125.30,125.05,124.60$, 123.27; IR (KBr, cm $\left.{ }^{-1}\right)$ 3629, 3300, 3061, 2360, 1599, 1496, 1441, 1381, 1254, 1110, 1097, 911, 771, 744, 703. MS: HR-ESI calculated for $\mathrm{C}_{60} \mathrm{H}_{37} \mathrm{O}_{4} \mathrm{P}[\mathrm{M}+\mathrm{Na}]:$ 875.2322, found: 875.2322. [ $\left.\alpha\right]_{\mathbf{D}}$ $=+119.1^{\circ}\left(\mathrm{c} 0.96, \mathrm{CH}_{2} \mathrm{Cl}_{2}\right)$

\section{Optimization of the DKR procedure (Tables 1-3).}

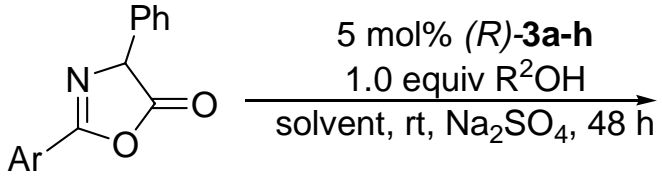

$( \pm)$

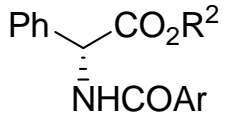

$(R)$ 
To a mixture of a freshly prepared azlactone substrate $(0.10 \mathrm{mmol})$, an alcohol indicated $(0.10$ $\mathrm{mmol})$ and $\mathrm{ca} .50 \mathrm{mg}$ of anhydrous sodium sulfate, was added a solution of a catalyst ( 0.005 $\mathrm{mmol}$ ) in $1 \mathrm{~mL}$ of a solvent. The reaction mixture was stirred at room temperature for 2 days, while the reaction progress was monitored by ${ }^{1} \mathrm{H}$ NMR and/or TLC. After the reaction was complete, the mixture was applied directly to a silica gel column and eluted with hexane $/ \mathrm{CH}_{2} \mathrm{Cl}_{2} / \mathrm{EtOAc}$ (50:15:15). Enantiomeric excess was determined by direct HPLC analysis of the ester product (for conditions, see Section 7.)

\section{Comparison of untreated and activated forms of catalyst $3 c$.}<smiles>O=C1OC([Al])=NC1c1ccccc1</smiles>

(士)

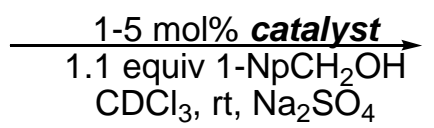

$\mathrm{Ar}=3,5-(\mathrm{MeO})_{2} \mathrm{C}_{6} \mathrm{H}_{3}$<smiles>O=C(N[C@@H](C(=O)OCc1ccccc1)c1ccccc1)c1ccccc1</smiles>

(R)

Table 1S.

\begin{tabular}{ccccc}
\hline Time (h) & $\begin{array}{c}\text { Untreated } \\
\text { catalyst, } \mathbf{5 ~ m o l} \%\end{array}$ & $\begin{array}{c}\text { Catalyst washed } \\
\text { w/HCl, } \mathbf{5 ~ m o l} \%\end{array}$ & $\begin{array}{c}\text { Catalyst washed } \\
\text { w/HCl, 2 mol \% }\end{array}$ & $\begin{array}{c}\text { Catalyst washed } \\
\text { w/HCl, 1 mol\% }\end{array}$ \\
\hline 0.5 & 4 & 52 & 25 & 16 \\
1 & 8 & 57 & 39 & 24 \\
2 & 14 & 75 & 53 & 33 \\
3 & 22 & 81 & 59 & 39 \\
4 & 26 & 87 & 66 & 47 \\
5 & 35 & 90 & 69 & 50 \\
6 & 37 & 92 & 75 & 57 \\
12 & 66 & 100 & 86 & 73 \\
24 & 82 & - & 100 & 87 \\
36 & 92 & - & - & - \\
48 & 100 & - & - & 100 \\
\hline
\end{tabular}

Untreated catalyst. Catalyst 3c was purified by chromatography $\left(\mathrm{CH}_{2} \mathrm{Cl}_{2} / \mathrm{MeOH}=100: 2\right)$. Solvent removal left yellow, compact residue, which was taken up in $\mathrm{CH}_{2} \mathrm{Cl}_{2}$, diluted with hexanes and evaporated again to dryness to produce flocculent, off-white powder.

Acid-washed catalyst. A solution of the purified catalyst $3 \mathrm{c}$ in $\mathrm{CH}_{2} \mathrm{Cl}_{2}$ (see above) was shaken in a separatory funnel with $4 \mathrm{M}$ aqueous $\mathrm{HCl}$, separated from the aqueous layer, dried over $\mathrm{Na}_{2} \mathrm{SO}_{4}$, diluted with hexane and evaporated to dryness. 
Catalytic activity measurements. Stock solutions of both forms of catalyst $3 \mathrm{c}$ in $\mathrm{CDCl}_{3}(0.005 \mathrm{M})$ were prepared. These were added separately to identically prepared mixtures of azlactone 1a $(0.10 \mathrm{mmol})$, 1-naphthylmethanol $(0.10 \mathrm{mmol})$, and $\mathrm{Na}_{2} \mathrm{SO}_{4}$. The solutions were transferred into NMR tubes and checked by ${ }^{1} \mathrm{H}$ NMR at time intervals indicated below, monitoring peaks at $\delta 5.5$ ppm (singlet, starting material) and $\delta 5.8 \mathrm{ppm}$ (doublet, product). The test was conducted analogously with lower catalyst loadings of acid-washed catalyst 3c (using $0.002 \mathrm{M}$ and $0.001 \mathrm{M}$ stock solutions, respectively). In each case, enantiomeric purity of the product was determined. The data shown in on the graph in Figure 2 are listed in Table 1S.

\section{Optimized DKR procedure (Table 4).}<smiles>[R]C1N=C([Te])OC1=O</smiles>

$( \pm)$

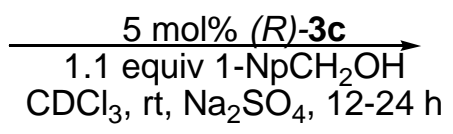

$\mathrm{Ar}=3,5-(\mathrm{MeO}){ }_{2} \mathrm{C}_{6} \mathrm{H}_{3}$<smiles>[R]C(N)C(=O)OCCN[NH3+]</smiles>

$(R)$

To a mixture of a freshly prepared azlactone substrate $(0.10 \mathrm{mmol}), 1$-naphthylmethanol $(0.11$ mmol), and ca. $50 \mathrm{mg}$ of $\mathrm{Na}_{2} \mathrm{SO}_{4}$. was added a solution of catalyst 3c $(0.005 \mathrm{mmol}$, washed $w / \mathrm{HCl}$ ) in $1 \mathrm{~mL}$ of $\mathrm{CHCl}_{3}$. After $12-24 \mathrm{~h}$, the mixture was applied directly to a silica gel column and eluted with hexane/ $\mathrm{CH}_{2} \mathrm{Cl}_{2} /$ EtOAc (10:3:3). Enantiomeric excess of the ester products was determined either directly by HPLC analysis or (for $\mathrm{R}^{1}=i$-Pr) after LAH reduction to the alcohol.

\section{Absolute configuration of the products.}

Absolute configuration of the major enantiomer was determined in the case of esters with $\mathrm{R}^{1}=$ $\mathrm{Ph}, \mathrm{Me}$ and $i$-Pr by HPLC comparison with authentic samples derived from commercially available amino acids. In the case of other e with $\mathrm{R}^{1}=$ Aryl, the absolute configuration was assigned by analogy with $\mathrm{R}^{1}=\mathrm{Ph}$.

\section{Characterization data and HPLC properties}<smiles>O=C(NC(C(=O)OCc1ccccc1)c1ccccc1)c1ccccc1</smiles>

${ }^{1}$ HNMR $\left(300 \mathrm{MHz}, \mathrm{CDCl}_{3}\right): \delta$ 7.74-7.71 (m, 2H), 7.44-7.10 (m, $13 \mathrm{H}), 5.75(\mathrm{~d}, J=7.2 \mathrm{~Hz}, 1 \mathrm{H}), 5.13\left(\mathrm{~A}\right.$ of $\mathrm{AB}, J_{A B}=12.6 \mathrm{~Hz}, 1 \mathrm{H}$ ), 5.09 (B of $\left.\mathrm{AB}, J_{A B}=12.6 \mathrm{~Hz}, 1 \mathrm{H}\right) ;{ }^{13} \mathrm{C}$ NMR $\left(75 \mathrm{MHz}, \mathrm{CDCl}_{3}\right)$ : $\delta 171.13,166.79,136.75,135.34,133.88,132.10,129.23,128.84$, 128.77, 128.61, 128.18, 127.62, 127.41, 67.77, 57.17; IR(thin film): 3319, 3062, 1742, 1644, 1520, 1485, 1327, 1171, 696; MS: HR-ESI calculated for $\mathrm{C}_{22} \mathrm{H}_{19} \mathrm{NO}_{3} \mathrm{H}\left(\mathrm{M}+\mathrm{H}^{+}\right) \mathrm{m} / \mathrm{z}$ : 346.1445, found: $\mathrm{m} / \mathrm{z}: 346.1438$. HPLC (CHIRALCEL OD-H, IPA/hexane=3/10, $1.0 \mathrm{~mL} / \mathrm{min}$ ): (R)-enantiomer: $27.1 \mathrm{~min}$; (S)enantiomer: $33.8 \mathrm{~min}$.<smiles>O=C(NC(C(=O)OCc1ccccc1)c1ccccc1)c1ccc(Cl)cc1</smiles>

${ }^{1}$ HNMR (300 MHz, $\left.\mathrm{CDCl}_{3}\right): \delta$ 7.77-7.74 (m, 2H), 7.42-7.28 $(\mathrm{m}, 9 \mathrm{H}), 7.23-7.19(\mathrm{~m}, 2 \mathrm{H}), 7.13(\mathrm{~d}, J=6.6 \mathrm{~Hz}, 1 \mathrm{H}), 5.80(\mathrm{~d}$, $J=6.9 \mathrm{~Hz}, 1 \mathrm{H}$ ), 5.23 (A of AB, $\left.J_{A B}=12.6 \mathrm{~Hz}, 1 \mathrm{H}\right), 5.18$ (B of $\left.\mathrm{AB}, J_{A B}=12.6 \mathrm{~Hz}, 1 \mathrm{H}\right) ;{ }^{13} \mathbf{C} \mathbf{N M R}\left(75 \mathrm{MHz}, \mathrm{CDCl}_{3}\right): \delta$ $171.02,165.71,138.40,136.53,135.23,132.23,129.26$, $129.11,128.90,128.82,128.77,128.64,128.17,127.58$, 100.08, 67.85, 67.20; IR (KBr, cm $\left.{ }^{-1}\right) 3323,3033,2922,1739$, 
1639, 1525, 1484, 1172, 696; MS: HR-ESI calculated for $\mathrm{C}_{22} \mathrm{H}_{18} \mathrm{ClNO}_{3} \mathrm{H}\left(\mathrm{M}+\mathrm{H}^{+}\right) \mathrm{m} / \mathrm{z}$ : 380.1048, found $\mathrm{m} / \mathrm{z}$ : 380.1035. HPLC (CHIRALCEL OD-H, IPA/hexane=3/10, $1.0 \mathrm{~mL} / \mathrm{min}$ ): (R)enantiomer: $8.8 \mathrm{~min}$; (S)-enantiomer: $19.8 \mathrm{~min}$.<smiles>COc1ccc(C(=O)NC(C(=O)OCc2ccccc2)c2ccccc2)cc1</smiles>

${ }^{1}$ HNMR (300 MHz, $\left.\mathrm{CDCl}_{3}\right): \delta 7.79(\mathrm{~d}, J=8.7 \mathrm{~Hz}, 2 \mathrm{H})$, 7.43-7.29 (m, 8H), 7.22-7.19 (m, 2H), 7.23-7.19 (m, 2H), $6.92(\mathrm{~d}, J=8.7 \mathrm{~Hz}, 2 \mathrm{H}), 7.13(\mathrm{~d}, J=6.6 \mathrm{~Hz}, 1 \mathrm{H}), 5.82(\mathrm{~d}$, $J=6.9 \mathrm{~Hz}, 1 \mathrm{H}), 5.22\left(\mathrm{~A}\right.$ of $\left.\mathrm{AB}, J_{A B}=12.6 \mathrm{~Hz}, 1 \mathrm{H}\right), 5.17$ (B of $\left.\mathrm{AB}, J_{A B}=12.6 \mathrm{~Hz}, 1 \mathrm{H}\right), 3.84(\mathrm{~s}, 3 \mathrm{H}) ;{ }^{13} \mathrm{C}$ NMR $(75 \mathrm{MHz}$, $\left.\mathrm{CDCl}_{3}\right): \delta 171.25,166.29,162.72,136.90,135.36,129.24$ $129.18,128.74,128.57,128.14,127.59,126.14,114.02,67.71,57.11,55.64 ; \mathbf{I R}\left(\mathrm{KBr}, \mathrm{cm}^{-1}\right)$ 3317, 2922, 1741, 1641, 1606, 1496, 1254, 1175, 697. MS: HR-ESI calculated for $\mathrm{C}_{23} \mathrm{H}_{21} \mathrm{NO}_{4} \mathrm{H}$ $\left(\mathrm{M}+\mathrm{H}^{+}\right) \mathrm{m} / \mathrm{z}: 376.1543$, found $\mathrm{m} / \mathrm{z}: 376.1536$. HPLC (CHIRALCEL OD-H, IPA/hexane=3/10, $1.0 \mathrm{~mL} / \mathrm{min}$ ): (R)-enantiomer: $11.9 \mathrm{~min}$; $(\mathrm{S})$-enantiomer: $24.6 \mathrm{~min}$.<smiles>COc1cc(OC)cc(C(=O)NC(C(=O)OCc2ccccc2)c2ccccc2)c1</smiles>

${ }^{1}$ HNMR $\left(300 \mathrm{MHz}, \mathrm{CDCl}_{3}\right): \delta 7.43-7.26(\mathrm{~m}, 8 \mathrm{H}), 7.23-$ $7.16(\mathrm{~m}, 2 \mathrm{H}), 6.94-6.93(\mathrm{~m}, 2 \mathrm{H}), 6.59-6.57(\mathrm{~m}, 1 \mathrm{H}), 5.80(\mathrm{~d}$, $J=6.9 \mathrm{~Hz}, 1 \mathrm{H}), 5.22\left(\mathrm{~A}\right.$ of $\left.\mathrm{AB}, J_{A B}=12.6 \mathrm{~Hz}, 1 \mathrm{H}\right), 5.17(\mathrm{~B}$ of $\left.\mathrm{AB}, J_{A B}=12.6 \mathrm{~Hz}, 1 \mathrm{H}\right), 3.80(\mathrm{~s}, 6 \mathrm{H}) ;{ }^{13} \mathrm{C}$ NMR $(75$ $\left.\mathrm{MHz}, \quad \mathrm{CDCl}_{3}\right): \delta 170.99, \quad 166.65,161.13,136.63$, $136.05,135.32,129.21,128.82,128.76,128.59,128.16$, 127.61, 105.32, 104.23, 67.75, 57.24, 55.81. IR (KBr, $\mathrm{cm}^{-}$ 1) $3315,2923,1743,1648,1594,1521,1205,1157,697$;MS: HR-ESI calculated for $\mathrm{C}_{24} \mathrm{H}_{23} \mathrm{NO}_{5} \mathrm{H}$ $\left(\mathrm{M}+\mathrm{H}^{+}\right) \mathrm{m} / \mathrm{z}:$ 406.1649, found m/z: 406.1643. HPLC (CHIRALCEL OD-H, IPA/hexane= 3/10, $1.0 \mathrm{~mL} / \mathrm{min}$ ): (S)-enantiomer: $18.9 \mathrm{~min}$; (R)-enantiomer: $28.0 \mathrm{~min}$.<smiles>COC(=O)C(NC(=O)c1cc(OC)cc(OC)c1)c1ccccc1</smiles>

${ }^{1}$ HNMR $\left(300 \mathrm{MHz}, \mathrm{CDCl}_{3}\right): \delta 7.45-7.34(\mathrm{~m}, 5 \mathrm{H}), 7.12(\mathrm{~d}, J=$ $6.6 \mathrm{~Hz}, 1 \mathrm{H}), 6.94-6.93(\mathrm{~m}, 2 \mathrm{H}), 6.59-6.57(\mathrm{~m}, 1 \mathrm{H}), 5.74(\mathrm{~d}, J=$ $6.9 \mathrm{~Hz}, 1 \mathrm{H}), 3.81(\mathrm{~s}, 6 \mathrm{H}), 3.76(\mathrm{~s}, 3 \mathrm{H}) .{ }^{13} \mathrm{C}$ NMR $(75 \mathrm{MHz}$, $\left.\mathrm{CDCl}_{3}\right): \delta 171.82,166.82,161.33,136.90,136.22,129.46$, $129.06,127.76,105.52,104.37,57.31,56.02,53.33$; IR $(\mathrm{KBr}$, $\mathrm{cm}^{-1}$ ) 3315, 2923, 1743, 1648, 1594, 1521, 1205, 1157, 697; MS: HR-ESI calculated for $\mathrm{C}_{18} \mathrm{H}_{19} \mathrm{NO}_{5} \mathrm{H}\left(\mathrm{M}+\mathrm{H}^{+}\right) \mathrm{m} / \mathrm{z}$ : 330.1336, found $\mathrm{m} / \mathrm{z}$ : 330.1327. HPLC (CHIRALCEL OD-H, IPA/hexane=3/10, $1.0 \mathrm{~mL} / \mathrm{min})$ : $(\mathrm{S})$-enantiomer: $15.6 \mathrm{~min}$; $(\mathrm{R})$-enantiomer: $18.1 \mathrm{~min}$.<smiles>COc1cc(OC)cc(C(=O)NC(C(=O)OC(C)C)c2ccccc2)c1</smiles>

${ }^{1}$ HNMR $\left(300 \mathrm{MHz}, \mathrm{CDCl}_{3}\right): \delta 7.44-7.25(\mathrm{~m}, 5 \mathrm{H}), 7.14(\mathrm{~d}, J=$ $6.9 \mathrm{~Hz}, 1 \mathrm{H}), 6.94-6.93(\mathrm{~m}, 2 \mathrm{H}), 6.59-6.57(\mathrm{~m}, 1 \mathrm{H}), 5.69(\mathrm{~d}, J=$ $6.6 \mathrm{~Hz}, 1 \mathrm{H}), 5.12-5.04(\mathrm{~m}, 1 \mathrm{H}), 3.81(\mathrm{~s}, 6 \mathrm{H}), 1.29(\mathrm{~d}, J=6.3 \mathrm{~Hz}$, $3 \mathrm{H}), 1.12(\mathrm{~d}, J=6.0 \mathrm{~Hz}, 3 \mathrm{H}) ;{ }^{13} \mathrm{C}$ NMR $\left(75 \mathrm{MHz}, \mathrm{CDCl}_{3}\right): \delta$ $170.63,166.55,161.12,137.03,136.21,129.11,128.63,127.43$, $105.29,104.15,70.09,57.20,55.82,21.94,21.57 ; \mathbf{I R}\left(\mathrm{KBr}, \mathrm{cm}^{-\mathbf{1}}\right)$ $3319,2922,1737,1651,1594,1520,1456,1354,1206,1157$, 1105, 1064, 698; MS: HR-ESI calculated for $\mathrm{C}_{20} \mathrm{H}_{23} \mathrm{NO}_{5} \mathrm{H}\left(\mathrm{M}+\mathrm{H}^{+}\right) \mathrm{m} / \mathrm{z}$ : 358.1649 , found m/z: 358.1636. HPLC (CHIRALCEL AS-H, IPA/hexane=2/10, $1.0 \mathrm{~mL} / \mathrm{min}$ ): (S)-enantiomer: 11.3 min; (R)-enantiomer: 14.2 min. 


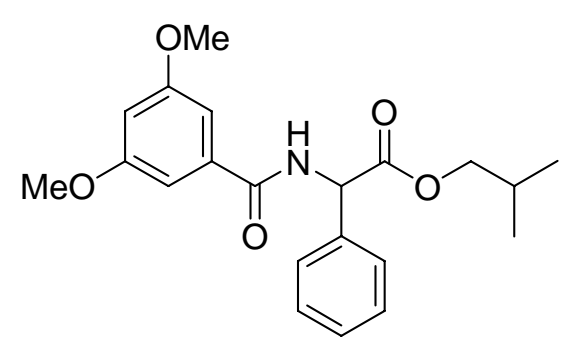

${ }^{1}$ HNMR $\left(300 \mathrm{MHz}, \mathrm{CDCl}_{3}\right): \delta 7.45-7.42(\mathrm{~m}, 2 \mathrm{H}), 7.39-7.29$ $(\mathrm{m}, 3 \mathrm{H}), 7.19(\mathrm{~d}, J=6.6 \mathrm{~Hz}, 1 \mathrm{H}), 6.95-6.94(\mathrm{~m}, 2 \mathrm{H}), 6.59-$ $6.57(\mathrm{~m}, 1 \mathrm{H}), 5.74(\mathrm{~d}, J=6.9 \mathrm{~Hz}, 1 \mathrm{H}), 3.96-3.93(\mathrm{~m}, 2 \mathrm{H})$, $3.81(\mathrm{~s}, 6 \mathrm{H}), 1.98-1.80(\mathrm{~m}, 1 \mathrm{H}), 0.82(\mathrm{~m}, 6 \mathrm{H}) ;{ }^{13} \mathrm{C}$ NMR $(75$ $\left.\mathrm{MHz}, \mathrm{CDCl}_{3}\right): \delta 171.20,166.59,161.13,137.01,136.13$, $129.14,128.72,127.47,105.30,104.18,72.12,57.20,55.80$, 27.88, 19.04; IR $\left(\mathrm{KBr}, \mathrm{cm}^{-1}\right) 3316,2961,1741,1651,1595$, 1522, 1456, 1353, 1206, 1157, 1065, 699; MS: HR-ESI calculated for $\mathrm{C}_{21} \mathrm{H}_{25} \mathrm{NO}{ }_{5} \mathrm{H}\left(\mathrm{M}+\mathrm{H}^{+}\right) \mathrm{m} / \mathrm{z}$ : 372.1805, found $\mathrm{m} / \mathrm{z}$ : 372.1808. HPLC (CHIRALCEL OD-H, IPA/hexane=3/10, $1.0 \mathrm{~mL} / \mathrm{min}$ ): (S)-enantiomer: $21.3 \mathrm{~min}$; (R)enantiomer: $34.6 \mathrm{~min}$.<smiles>COc1cc(OC)cc(C(=O)NC(C(=O)OCC(C)(C)C)c2ccccc2)c1</smiles>

${ }^{1}$ HNMR (300 MHz, $\left.\mathrm{CDCl}_{3}\right): \delta$ 7.46-7.43 (m, 2H), 7.39-7.31 $(\mathrm{m}, 3 \mathrm{H}), 7.21(\mathrm{~d}, J=6.9 \mathrm{~Hz}, 1 \mathrm{H}), 6.96-6.95(\mathrm{~m}, 2 \mathrm{H}), 6.59-$ $6.58(\mathrm{~m}, 1 \mathrm{H}), 5.75(\mathrm{~d}, J=6.9 \mathrm{~Hz}, 1 \mathrm{H}), 3.91(\mathrm{~A}$ of $\mathrm{AB}$ $\left.J_{A B}=10.5 \mathrm{~Hz}, 1 \mathrm{H}\right), 3.80\left(\mathrm{~B}\right.$ of $\left.\mathrm{AB} J_{A B}=10.5 \mathrm{~Hz}, 1 \mathrm{H}\right),-3.81(\mathrm{~s}$, $6 \mathrm{H}), 0.82(\mathrm{~s}, 6 \mathrm{H}) ;{ }^{13} \mathrm{C}$ NMR $\left(75 \mathrm{MHz}, \mathrm{CDCl}_{3}\right): \delta$ 171.20, $166.58,161.14,137.09,136.15,129.12$, 128.71, 127.43, $105.30,104.18,75.27,57.25,55.82,31.72,26.41$; IR $(\mathrm{KBr}$, $\mathrm{cm}^{-1}$ ) 3318, 2958, 1741, 1648, 1594, 1522, 1205, 1157, 698; MS: HR-ESI calculated for $\mathrm{C}_{22} \mathrm{H}_{27} \mathrm{NO}_{5} \mathrm{H}\left(\mathrm{M}+\mathrm{H}^{+}\right) \mathrm{m} / \mathrm{z}$ : 386.1962, found $\mathrm{m} / \mathrm{z}$ : 386.1968; HPLC (CHIRALCEL OD-H, IPA/hexane=3/10, $1.0 \mathrm{~mL} / \mathrm{min}$ ): (S)-enantiomer: $15.6 \mathrm{~min}$; (R)enantiomer: $39.9 \mathrm{~min}$.<smiles>C=CCOC(=O)C(NC(=O)c1cc(OC)cc(OC)c1)c1ccccc1</smiles>

${ }^{1}$ HNMR $\left(300 \mathrm{MHz}, \mathrm{CDCl}_{3}\right): \delta$ 7.46-7.31 (m, 5H), $7.13(\mathrm{~d}$, $J=6.9 \mathrm{~Hz}, 1 \mathrm{H}), 6.94-6.93(\mathrm{~m}, 2 \mathrm{H}), 6.59-6.57(\mathrm{~m}, 1 \mathrm{H})$, 5.91-5.79 (m, 1H), $5.76(\mathrm{~d}, J=6.9 \mathrm{~Hz}, 1 \mathrm{H}), 5.24-20(\mathrm{~m}$, $1 \mathrm{H}), 5.18-5.17(\mathrm{~m}, 1 \mathrm{H}) 4.68-4.64(\mathrm{~m}, 2 \mathrm{H}), 3.81(\mathrm{~s}, 6 \mathrm{H}) ;{ }^{13} \mathrm{C}$ NMR $\left(75 \mathrm{MHz}, \mathrm{CDCl}_{3}\right): \delta 170.83,166.62,161.13,136.69$, $136.05,131.47,129.24,128.85,118.94,105.31,104.19$, 66.54, 57.19, 55.81. IR (KBr, cm $\left.{ }^{-1}\right) 3313,2921,1742,1646$, 1594, 1521, 1455, 1352, 1205, 1157, 1054, 699; MS: HR-

ESI calculated for $\mathrm{C}_{20} \mathrm{H}_{21} \mathrm{NO}_{5} \mathrm{H}\left(\mathrm{M}+\mathrm{H}^{+}\right) \mathrm{m} / \mathrm{z}$ : 356.1492, found $\mathrm{m} / \mathrm{z}$ : 356.1483; HPLC (CHIRALCEL OD-H, IPA/hexane=3/10, $1.0 \mathrm{~mL} / \mathrm{min}$ ): (R)-enantiomer: $31.8 \mathrm{~min}$; (S)enantiomer: $58.8 \mathrm{~min}$.<smiles>COc1cc(OC)cc(C(=O)NC(C(=O)OCc2ccc(Br)cc2)c2ccccc2)c1</smiles>

${ }^{1}$ HNMR (300 MHz, $\left.\mathrm{CDCl}_{3}\right): \delta 7.43-7.33(\mathrm{~m}, 7 \mathrm{H})$, 7.11-7.04 (m, 3H), 6.93-6.92 (m, 2H), 6.59-6.58 (m, $1 \mathrm{H}), 5.76(\mathrm{~d}, J=6.9 \mathrm{~Hz}, 1 \mathrm{H}), 5.13$ (apparent s, 2H), $3.80(\mathrm{~s}, 6 \mathrm{H}) ;{ }^{13} \mathrm{C}$ NMR $\left(75 \mathrm{MHz}, \mathrm{CDCl}_{3}\right): \delta 170.88$, 166.70, 161.14, 136.40, 135.94, 134.36, 131.91, 129.76, 129.29, 128.96, 127.61, 122.64, 105.32,104.20, 66.86, 57.27, 55.82; IR (KBr, cm $\left.{ }^{-1}\right)$ 3315, 2922, 1744, 1652, 1594, 1519, 1455, 1352, 1205, 1157, 1069, 698; MS: 
HR-ESI calculated for $\mathrm{C}_{24} \mathrm{H}_{22} \mathrm{BrNO}_{5} \mathrm{H}\left(\mathrm{M}+\mathrm{H}^{+}\right) \mathrm{m} / \mathrm{z}$ : 484.0754, found $\mathrm{m} / \mathrm{z}$ : 484.0735; HPLC (CHIRALCEL OD-H, IPA/hexane=3/10, $1.0 \mathrm{~mL} / \mathrm{min}$ ): (S)-enantiomer: $24.4 \mathrm{~min}$; (R)enantiomer: $41.7 \mathrm{~min}$.<smiles>COc1ccc(COC(=O)C(NC(=O)c2cc(OC)cc(OC)c2)c2ccccc2)cc1</smiles>

${ }^{1}$ HNMR $\left(300 \mathrm{MHz}, \mathrm{CDCl}_{3}\right): \delta 7.40-7.32(\mathrm{~m}, 4 \mathrm{H})$, 7.19-7.12 (m, 3H), 6.85-6.82 (m, 2H), 6.59-6.57 (m, $1 \mathrm{H}), 5.17\left(\mathrm{~A}\right.$ of $\left.\mathrm{AB}, J_{A B}=12.0 \mathrm{~Hz}, 1 \mathrm{H}\right), 5.10(\mathrm{~B}$ of $\left.\mathrm{AB}, J_{A B}=12.0 \mathrm{~Hz}, 1 \mathrm{H}\right), 3.81(\mathrm{~s}, 6 \mathrm{H}), 3.79(\mathrm{~s}, 3 \mathrm{H}) ;{ }^{13} \mathrm{C}$ NMR $\left(75 \mathrm{MHz}, \mathrm{CDCl}_{3}\right): \delta 171.23,166.59,161.13$, $159.97,136.71,136.09,130.13,129.17,128.76$ $127.56,114.14,105.30,104.20,67.71,57.19,55.80$ 55.48. IR $\left(\mathrm{KBr}, \mathrm{cm}^{-1}\right) 3316,2918,2849,1739,1653$, 1593, 1514, 1456, 1352, 1248, 1205, 1157, 1064, 1032, 822, 699; MS: HR-ESI calculated for $\mathrm{C}_{25} \mathrm{H}_{25} \mathrm{NO}_{6} \mathrm{H}\left(\mathrm{M}+\mathrm{H}^{+}\right) \mathrm{m} / \mathrm{z}:$ 436.1755, found $\mathrm{m} / \mathrm{z}$ : 436.1769. HPLC (CHIRALCEL OD-H, IPA/hexane $=2 / 10,1.0 \mathrm{~mL} / \mathrm{min}$ ): (S)-enantiomer: 27.4 min; (R)-enantiomer: 36.4 min.<smiles>COc1cc(OC)cc(C(=O)NC(C(=O)OCc2c(F)c(F)c(F)c(F)c2F)c2ccccc2)c1</smiles>

${ }^{1}$ HNMR $\left(300 \mathrm{MHz}, \mathrm{CDCl}_{3}\right): \delta 7.38-7.33(\mathrm{~m}, 5 \mathrm{H}), 6.97$ $(\mathrm{d}, J=6.6 \mathrm{~Hz}, 1 \mathrm{H}), 6.92-6.91(\mathrm{~m}, 2 \mathrm{H}), 6.59-6.58(\mathrm{~m}$, $1 \mathrm{H}), 5.73(\mathrm{~d}, J=6.9 \mathrm{~Hz}, 1 \mathrm{H}), 5.28$ (apparent s, 2H), $3.81(\mathrm{~s}, 6 \mathrm{H}) .{ }^{13} \mathrm{C}$ NMR $\left(75 \mathrm{MHz}, \mathrm{CDCl}_{3}\right): \delta 170.50$, $166.75,161.15,135.84,135.80,129.29,129.08,127.48$ 105.31, 104.22, 57.16, 55.81, 54.72. IR $\left(\mathrm{KBr}, \mathrm{cm}^{-1}\right)$ 3316, 2921, 1751, 1657, 1594, 1523, 1508, 1456, 1353, 1206, 1157, 1061, 941, 698; MS: HRESI calculated for $\mathrm{C}_{24} \mathrm{H}_{18} \mathrm{~F}_{5} \mathrm{NO}_{5} \mathrm{H}\left(\mathrm{M}+\mathrm{H}^{+}\right) \mathrm{m} / \mathrm{z}$ : 496.1178, found $\mathrm{m} / \mathrm{z}: 496.1172$. HPLC (CHIRALCEL AD-H, IPA/hexan=2/10,1.0 mL/min): (S)-enantiomer: $15.3 \mathrm{~min}$; (R)-enantiomer: $31.3 \mathrm{~min}$.<smiles>COc1cc(OC)cc(C(=O)NC(C(=O)OCc2cccc3ccccc23)c2ccccc2)c1</smiles>

${ }^{1}$ HNMR $\left(300 \mathrm{MHz}, \mathrm{CDCl}_{3}\right): \delta$ 7.87-7.82 (m, 2H), 7.77$7.74(\mathrm{~m}, 1 \mathrm{H}), 7.51-7.37(\mathrm{~m}, 6 \mathrm{H}), 7.32-7.28(\mathrm{~m}, 3 \mathrm{H}), 7.2$ $(\mathrm{d}, J=6.9 \mathrm{~Hz}, 1 \mathrm{H}), 6.95-6.93(\mathrm{~m}, 2 \mathrm{H}), 6.59-6.58(\mathrm{~m}$, $1 \mathrm{H}), 5.79(\mathrm{~d}, J=6.9 \mathrm{~Hz}, 1 \mathrm{H}), 5.64(\mathrm{~s}, 2 \mathrm{H}), 3.78(\mathrm{~s}, 6 \mathrm{H})$; ${ }^{13} \mathrm{C}$ NMR $\left(75 \mathrm{MHz}, \mathrm{CDCl}_{3}\right): \delta 171.04,166.68,161.13$, $136.52, \quad 136.05,133.88,131.67, \quad 130.72,129.76$, $129.18128 .85,128.80,127.68,127.58,126.82,126.19$, 125.36,123.63, 105.33, 104.24, 66.46, 57.34, 55.82; IR $\left(\mathrm{KBr}, \mathrm{cm}^{-1}\right) 3327,2920,1740,1651,1594,1512,1456,1353,1263,1205,1156,1063,1049,792$, 776; MS: HR-ESI calculated for $\mathrm{C}_{28} \mathrm{H}_{25} \mathrm{NO}_{5} \mathrm{H}\left(\mathrm{M}+\mathrm{H}^{+}\right) \mathrm{m} / \mathrm{z}$ : 456.1805, found $\mathrm{m} / \mathrm{z}$ : 456.1814 . HPLC (CHIRALCEL OD-H, IPA/hexane=3/10, $1.0 \mathrm{~mL} / \mathrm{min}$ ): (S)-enantiomer: $24.8 \mathrm{~min}$; (R)enantiomer: $46.1 \mathrm{~min}$.<smiles>COc1cc(OC)cc(C(=O)NC(C(=O)OCc2ccc3ccccc3c2)c2ccccc2)c1</smiles>

${ }^{1}$ HNMR $\left(300 \mathrm{MHz}, \mathrm{CDCl}_{3}\right): \delta 7.83-7.72(\mathrm{~m}, 3 \mathrm{H})$, $7.62(\mathrm{~s}, 1 \mathrm{H}), 7.51-7.42(\mathrm{~m}, 4 \mathrm{H}), 7.36-7.34(\mathrm{~m}, 3 \mathrm{H})$, 7.31-7.28 (m, 1H), $7.15(\mathrm{~d}, J=6.6 \mathrm{~Hz}, 1 \mathrm{H}), 6.95-6.94$ $(\mathrm{m}, 2 \mathrm{H}), 6.59-6.58(\mathrm{~m}, 1 \mathrm{H}), 5.83(\mathrm{~d}, J=6.9 \mathrm{~Hz}, 1 \mathrm{H})$, $5.34(\mathrm{~m}, 2 \mathrm{H}), 3.80(\mathrm{~s}, 6 \mathrm{H}) ;{ }^{13} \mathbf{C}$ NMR $(75 \mathrm{MHz}$, $\left.\mathrm{CDCl}_{3}\right): \delta 171.00,166.66,161.14,136.65,136.05$, 
$133.33,133.30,132.77,129.26,128.88,128.59,128.21,127.90,127.64,127.16,126.54,125.54$, 105.30, 104.23, 67.80, 57.30, 55.82; IR $\left(\mathrm{KBr}, \mathrm{cm}^{-1}\right) 3318,2920,1741,1652,1594,1510,1455$, 1353, 1205, 1156, 1064, 698; MS: HR-ESI calculated for $\mathrm{C}_{28} \mathrm{H}_{25} \mathrm{NO}_{5} \mathrm{H}\left(\mathrm{M}+\mathrm{H}^{+}\right) \mathrm{m} / \mathrm{z}$ : 456.1805, found $\mathrm{m} / \mathrm{z}$ : 456.1816. HPLC (CHIRALCEL OD-H, IPA/hexane=4/10, $1.0 \mathrm{~mL} / \mathrm{min}$ ): (S)enantiomer: $30.1 \mathrm{~min}$; (R)-enantiomer: $42.3 \mathrm{~min}$.<smiles>COc1cc(OC)cc(C(=O)NC(C(=O)OCc2ccc3ccc4cccc5ccc2c3c45)c2ccccc2)c1</smiles>

${ }^{1}$ HNMR $\left(300 \mathrm{MHz}, \mathrm{CDCl}_{3}\right): \delta 8.21-8.19(\mathrm{~m}, 2 \mathrm{H}), 8.11-$ $8.00(\mathrm{~m}, 6 \mathrm{H}), 7.93(\mathrm{~d}, J=7.8 \mathrm{~Hz}, 1 \mathrm{H}), 7.39-7.36(\mathrm{~m}$, $3 \mathrm{H}), 7.26-7.24(\mathrm{~m}, 2 \mathrm{H}), 7.13(\mathrm{~d}, J=6.9 \mathrm{~Hz}, 1 \mathrm{H}), 6.92-$ $6.91(\mathrm{~m}, 2 \mathrm{H}), 6.57-6.55(\mathrm{~m}, 1 \mathrm{H}), 5.89(\mathrm{~s}, 2 \mathrm{H}), 5.81(\mathrm{~d}, \mathrm{~J}$ $=6.9 \mathrm{~Hz}, 1 \mathrm{H}), 3.76(\mathrm{~s}, 6 \mathrm{H}) ;{ }^{13} \mathrm{C}$ NMR $\left(75 \mathrm{MHz}, \mathrm{CDCl}_{3}\right)$ : $\delta 171.06,166.66,161.10,136.49,136.04,132.11$, $131.38,130.86,129.73,129.17,128.78,128.45,128.17$, $128.02,127.84,127.54,126.32,125.77,125.74,125.02$, $125.76,124.70,122.90,195.29,104.20,66.68,57.39$, 55.79; IR $\left(\mathrm{KBr}, \mathrm{cm}^{-1}\right) 3329,2922,1734,1639,1593,1520,1455,1352,1260,1205,1156,1064$, 844, 750. MS: HR-ESI calculated for $\mathrm{C}_{34} \mathrm{H}_{27} \mathrm{NO}_{5} \mathrm{Na}\left(\mathrm{M}+\mathrm{Na}^{+}\right) \mathrm{m} / \mathrm{z}$ : 552.1781, found $\mathrm{m} / \mathrm{z}$ : 552.1805. HPLC (CHIRALCEL OD-H, IPA/hexane=4/10, $1.0 \mathrm{~mL} / \mathrm{min}$ ): (S)-enantiomer: 22.8 min; (R)-enantiomer: 40.7 min.<smiles>COc1cc(OC)cc(C(=O)NC(C(=O)OCc2cccc3ccccc23)c2ccc(Cl)cc2)c1</smiles>

${ }^{1}$ HNMR $\left(300 \mathrm{MHz}, \mathrm{CDCl}_{3}\right): \delta$ 7.87-7.83 (m, 2H), $7.69(\mathrm{~d}$, $J=8.1 \mathrm{~Hz}, 1 \mathrm{H}), 7.52-7.47(\mathrm{~m}, 4 \mathrm{H}), 7.43-7.37(\mathrm{~m}, 5 \mathrm{H})$, 7.28-7.18 (m, 5H), $6.91(\mathrm{~d}, J=2.4 \mathrm{~Hz}, 2 \mathrm{H}), 6.59-6.57(\mathrm{~m}$, $1 \mathrm{H}), 5.72(\mathrm{~d}, J=6.6 \mathrm{~Hz}, 1 \mathrm{H}), 5.65(\mathrm{~d}, J=12.3 \mathrm{~Hz}, 1 \mathrm{H})$, $5.60(\mathrm{~d}, J=12.3 \mathrm{~Hz}, 1 \mathrm{H}), 3.79(\mathrm{~s}, 6 \mathrm{H}) ;{ }^{13} \mathrm{C}$ NMR $(75$ $\left.\mathrm{MHz}, \mathrm{CDCl}_{3}\right): \delta 170.64,166.56,161.16,135.80,135.17$, $134.67,133.89,131.63,130.43,129.95,129.24,128.89$, $128.82,127.99,126.85,126.26,125.30,123.49,105.29$, 104.29, 66.77, 56.67, 55.81; IR (KBr, cm $\left.{ }^{-1}\right)$ 3299, 2923, 1740, 1644, 1594, 1513, 1492, 1352, 1205, 1157, 1091, 1064, 794, 775; MS: HR-ESI calculated for $\mathrm{C}_{28} \mathrm{H}_{24} \mathrm{ClNO}_{5} \mathrm{H}\left(\mathrm{M}+\mathrm{H}^{+}\right) \mathrm{m} / \mathrm{z}$ : 490.1416, found m/z: 490.1410. HPLC (CHIRALCEL OD-H, IPA/hexane=3/10, $1.0 \mathrm{~mL} / \mathrm{min})$ : (S)-enantiomer: $35.6 \mathrm{~min}$; $(\mathrm{R})$-enantiomer: $47.9 \mathrm{~min}$.<smiles>COc1ccc(C(NC(=O)c2cc(OC)cc(OC)c2)C(=O)OCc2cccc3ccccc23)cc1</smiles>

${ }^{1}$ HNMR $\left(300 \mathrm{MHz}, \mathrm{CDCl}_{3}\right): \delta 7.86-7.82(\mathrm{~m}, 2 \mathrm{H}), 7.77-$ $7.74(\mathrm{~m}, 1 \mathrm{H}), 7.51-7.38(\mathrm{~m}, 4 \mathrm{H}), 7.30-7.26(\mathrm{~m}, 2 \mathrm{H}), 7.07$ $(\mathrm{d}, J=6.6 \mathrm{~Hz}, 1 \mathrm{H}), 6.91(\mathrm{~d}, J=2.1 \mathrm{~Hz}, 2 \mathrm{H}), 6.82-6.77(\mathrm{~m}$, $2 \mathrm{H}), 6.57(\mathrm{t}, J=2.1 \mathrm{~Hz}, 1 \mathrm{H}), 5.70(\mathrm{~d}, J=6.9 \mathrm{~Hz}, 1 \mathrm{H}), 5.63$ $(\mathrm{s}, 2 \mathrm{H}), 3.79(\mathrm{~s}, 6 \mathrm{H}), 3.77(\mathrm{~s}, 3 \mathrm{H}) ;{ }^{13} \mathrm{C}$ NMR $(75 \mathrm{MHz}$, $\left.\mathrm{CDCl}_{3}\right): \delta 192.25,171.22,166.62,161.11,159.97,136.11$, $133.87,131.68,130.77,129.73,128.79,128.59,127.71$, $126.77,126.15,125.35,123.68,114.54,105.28,104.20$, 66.38, 56.77, 55.51; IR $\left(\mathrm{KBr}, \mathrm{cm}^{-1}\right) 3316,2924,1741$, 1653, 1594, 1512, 1458, 1353, 1249, 1205, 1177, 1157, 1063, 797; MS: HR-ESI calculated for $\mathrm{C}_{29} \mathrm{H}_{27} \mathrm{NO}{ }_{6} \mathrm{H}\left(\mathrm{M}+\mathrm{H}^{+}\right) \mathrm{m} / \mathrm{z}:$ 486.1911, found $\mathrm{m} / \mathrm{z}$ : 486.1934. HPLC (CHIRALCEL OD-H, IPA/hexane=3/10, $1.0 \mathrm{~mL} / \mathrm{min}$ ): (S)-enantiomer: $35.0 \mathrm{~min}$; (R)-enantiomer: $49.9 \mathrm{~min}$. 
<smiles>COc1cc(OC)cc(C(=O)NC(C(=O)OCc2cccc3ccccc23)c2cccc(OC)c2)c1</smiles>

${ }^{1}$ HNMR $\left(300 \mathrm{MHz}, \mathrm{CDCl}_{3}\right): \delta$ 7.87-7.82 (m, 2H), 7.77$7.75(\mathrm{~m}, 1 \mathrm{H}), 7.51-7.38(\mathrm{~m}, 4 \mathrm{H}), 7.26-7.18(\mathrm{~m}, 1 \mathrm{H}), 7.11$ $(\mathrm{d}, J=6.6 \mathrm{~Hz}, 1 \mathrm{H}), 6.97-6.81(\mathrm{~m}, 5 \mathrm{H}), 6.59-6.58(\mathrm{~m}, 1 \mathrm{H})$, $5.75(\mathrm{~d}, J=7.2 \mathrm{~Hz}, 1 \mathrm{H}), 5.64(\mathrm{~s}, 2 \mathrm{H}), 3.80(\mathrm{~s}, 6 \mathrm{H}), 3.66(\mathrm{~s}$, $3 \mathrm{H}) ;{ }^{13} \mathrm{C}$ NMR $\left(75 \mathrm{MHz}, \mathrm{CDCl}_{3}\right): \delta$ 170.90, 166.66, $161.13,160.17,137.88,136.04,133.88,131.68,130.72$, $130.20,129.78,128.83,127.78,126.84,126.19,125.35$ $123.64,119.69,114.63,112.96,105.31,104.24,66.49$, 57.26, 55.40; IR (KBr, cm $\left.{ }^{-1}\right) 3313,2923,1741,1651,1595,1513,1456,1353,1263,1205,1156$, 1063, 1049, 792, 776; MS: HR-ESI calculated for $\mathrm{C}_{29} \mathrm{H}_{27} \mathrm{NO}_{6} \mathrm{H}\left(\mathrm{M}+\mathrm{H}^{+}\right) \mathrm{m} / \mathrm{z}$ : 486.1911, found m/z: 486.1910. HPLC (CHIRALCEL OD-H, IPA/hexane=3/10, $1.0 \mathrm{~mL} / \mathrm{min}$ ): (S)-enantiomer: 26.5 min; (R)-enantiomer: 47.0 min.<smiles>COc1cc(OC)cc(C(=O)NC(C(=O)OCc2cccc3ccccc23)c2ccccc2Cl)c1</smiles>

${ }^{1}$ HNMR $\left(300 \mathrm{MHz}, \mathrm{CDCl}_{3}\right): \delta$ 7.85-7.76 (m, 3H), 7.49$7.36(\mathrm{~m}, 5 \mathrm{H}), 7.32-7.13(\mathrm{~m}, 4 \mathrm{H}), 6.92-6.91(\mathrm{~m}, 2 \mathrm{H}), 6.58-$ $6.56(\mathrm{~m}, 1 \mathrm{H}), 6.11(\mathrm{~d}, J=7.2 \mathrm{~Hz}, 1 \mathrm{H}), 5.66(\mathrm{~s}, 2 \mathrm{H}), 3.78(\mathrm{~s}$, $6 \mathrm{H}) ;{ }^{13} \mathrm{C}$ NMR $\left(75 \mathrm{MHz}, \mathrm{CDCl}_{3}\right): \delta$ 170.33, 166.59, $161.11,135.94,134.83,133.83,133.78,131.63,130.70$ $130.67,130.36,129.92,129.69,128.80,127.63,127.44$ $126.72,126.11,125.30,123.57,105.32,104.27,66.64$, 57.79, 55.67; IR (KBr, cm $\left.{ }^{-1}\right)$ 3315, 2924, 1743, 1651, 1594, 1512, 1456, 1352, 1205, 1157, 1063, 797, 754, 735; MS: HR-ESI calculated for $\mathrm{C}_{28} \mathrm{H}_{24} \mathrm{ClNO}_{5} \mathrm{H}$ $\left(\mathrm{M}+\mathrm{H}^{+}\right) \mathrm{m} / \mathrm{z}:$ 490.1416, found m/z: 490.1410; HPLC (CHIRALCEL OD-H, IPA/hexane=3/10, $1.0 \mathrm{~mL} / \mathrm{min}$ ): (S)-enantiomer: $32.6 \mathrm{~min}$; (R)-enantiomer: $79.1 \mathrm{~min}$.<smiles>COc1cc(OC)cc(C(=O)NC(C(=O)OCc2cccc3ccccc23)c2ccccc2OC)c1</smiles>

${ }^{1}$ HNMR $\left(300 \mathrm{MHz}, \mathrm{CDCl}_{3}\right): \delta 7.82(\mathrm{t}, J=8.1 \mathrm{~Hz}, 2 \mathrm{H})$, $7.73(\mathrm{~d}, J=8.1 \mathrm{~Hz}, 1 \mathrm{H}), 7.49-7.32(\mathrm{~m}, 5 \mathrm{H}), 7.27-7.20(\mathrm{~m}$, $2 \mathrm{H}), 6.95-6.88(\mathrm{~m}, 3 \mathrm{H}), 6.65(\mathrm{~d}, J=8.4 \mathrm{~Hz}, 1 \mathrm{H})$, 6.56$6.55(\mathrm{~m}, 1 \mathrm{H}), 5.96(\mathrm{~d}, J=8.1 \mathrm{~Hz}, 1 \mathrm{H}), 5.66(\mathrm{~A}$ of $\mathrm{AB}$, $\left.J_{A B}=12.3 \mathrm{~Hz}, 1 \mathrm{H}\right), 5.57\left(\mathrm{~B}\right.$ of $\left.\mathrm{AB}, J_{A B}=12.3 \mathrm{~Hz}, 1 \mathrm{H}\right), 3.78$ $(\mathrm{s}, 6 \mathrm{H}), 3.30(\mathrm{~s}, 3 \mathrm{H}) ;{ }^{13} \mathrm{C}$ NMR $\left(75 \mathrm{MHz}, \mathrm{CDCl}_{3}\right)$ : $\delta 171.04,166.73,161.03,157,10,136.61,133.84,131.76$, $131.34,131.12,129.96,129.40,128.68,127.72,126.66$, 126.03, 125.50,125.36, 123.81, 121.18, 111.03, 105.34, 103.94, 66.04, 55.76, 55.13, 54.49; IR $\left(\mathrm{KBr}, \mathrm{cm}^{-1}\right)$ 3353, 2922, 1743, 1659, 1594, 1512, 1495, 1461, 1351, 1252, 1205, 1147, 1063, 755; MS: HR-ESI calculated for $\mathrm{C}_{29} \mathrm{H}_{27} \mathrm{NO}_{6} \mathrm{H}\left(\mathrm{M}+\mathrm{H}^{+}\right) \mathrm{m} / \mathrm{z}$ : 486.1911, found $\mathrm{m} / \mathrm{z}$ : 486.1895. HPLC (CHIRALCEL OD-H, IPA/hexane=3/10, $1.0 \mathrm{~mL} / \mathrm{min}$ ): (S)-enantiomer: $26.9 \mathrm{~min}$; (R)enantiomer: $38.8 \mathrm{~min}$.<smiles>COc1cc(OC)cc(C(=O)NC(C(=O)OCc2cccc3ccccc23)c2cccc3ccccc23)c1</smiles>

${ }^{1}$ HNMR $\left(300 \mathrm{MHz}, \mathrm{CDCl}_{3}\right): \delta 8.16-8.13(\mathrm{~m}, 1 \mathrm{H}), 7.86-$ $7.78(\mathrm{~m}, 3 \mathrm{H}), 7.67(\mathrm{~d}, J=8.1 \mathrm{~Hz}, 1 \mathrm{H}), 7.49-7.24(\mathrm{~m}, 8 \mathrm{H})$, $7.05(\mathrm{~d}, J=7.5 \mathrm{~Hz}, 2 \mathrm{H}), 6.90-6.89(\mathrm{~m}, 2 \mathrm{H}), 6.56-6.52(\mathrm{~m}$, $2 \mathrm{H}), 5.66(\mathrm{~s}, 2 \mathrm{H}), 3.76(\mathrm{~s}, 6 \mathrm{H}) ;{ }^{13} \mathrm{C}$ NMR $(75 \mathrm{MHz}$, $\left.\mathrm{CDCl}_{3}\right): \delta 171.53,166.92,161.09,135.96,134.31,133.79$, $132.41,131.60,131.30,130.72,129.71,129.65,129.10$ 128.72 , 127.75, 127.25, 126.67, 126.35, 126.07, 125.37, 
125.27, 123.58, 123.44, 105.33, 104.27, 66.41, 55.80, 54.50; IR (KBr, cm $\left.{ }^{-1}\right) 3312,2922,1740$, 1653, 1594, 1511, 1457, 1349, 1205, 1194, 1156, 1063, 792, 776; MS: HR-ESI calculated for $\mathrm{C}_{32} \mathrm{H}_{27} \mathrm{NO}{ }_{5} \mathrm{H}\left(\mathrm{M}+\mathrm{H}^{+}\right) \mathrm{m} / \mathrm{z}$ : 506.1962, found $\mathrm{m} / \mathrm{z}$ : 506.1941. HPLC (CHIRALCEL OD-H, IPA/hexane=3/10, $1.0 \mathrm{~mL} / \mathrm{min}$ ): (S)-enantiomer: $22.9 \mathrm{~min}$; (R)-enantiomer: $66.6 \mathrm{~min}$.<smiles>COc1cc(OC)cc(C(=O)NC(C(=O)OCc2cccc3ccccc23)c2ccc3ccccc3c2)c1</smiles>

${ }^{1}$ HNMR (300 MHz, $\left.\mathrm{CDCl}_{3}\right): \delta$ 7.82-7.74 (m, 5H), 7.69 (d, $J=8.4 \mathrm{~Hz}, 1 \mathrm{H}), 7.51-7.34(\mathrm{~m}, 6 \mathrm{H}), 7.27-6.95(\mathrm{~m}, 2 \mathrm{H})$, 6.95-6.94 (m, 1H), 6.59-6.58 (m, 1H), $5.96(\mathrm{~d}, J=6.6 \mathrm{~Hz}$, 1H), $5.64(\mathrm{~s}, 2 \mathrm{H}), 3.79(\mathrm{~s}, 6 \mathrm{H}) ;{ }^{13} \mathrm{C}$ NMR $\left(75 \mathrm{MHz}^{\mathrm{C}} \mathrm{CDCl}_{3}\right)$ : $\delta 170.05,166.70,161.14,136.04,133.88,133.84,133.47$, $133.44,131.65,130.64,129.81,129.10,128.77,128.33$, $127.87,126.93,126.73,126.68,126.63,126.14,125.30$, $124.98,123.55,105.33,104.29,66.61,57.43,55.80$; IR $\left(\mathrm{KBr}, \mathrm{cm}^{-1}\right) 3313,2923,1741,1654,1595,1511,1458$, 1351, 1205, 1157, 1064, 793,776. MS: HR-ESI calculated for $\mathrm{C}_{32} \mathrm{H}_{27} \mathrm{NO}{ }_{5} \mathrm{H}\left(\mathrm{M}+\mathrm{H}^{+}\right) \mathrm{m} / \mathrm{z}$ : 506.1962, found m/z: 506.1939. HPLC (CHIRALCEL OD-H, IPA/hexane=3/10,1.0 mL/min): (S)-enantiomer: $27.1 \mathrm{~min}$; (R)-enantiomer: $50.7 \mathrm{~min}$.<smiles>COc1cc(OC)cc(C(=O)NC(C)C(=O)OCc2cccc3ccccc23)c1</smiles>

${ }^{1}$ HNMR $\left(300 \mathrm{MHz}, \mathrm{CDCl}_{3}\right): \delta 8.01-7.98(\mathrm{~m}, 1 \mathrm{H}), 7.91-$ $7.86(\mathrm{~m}, 2 \mathrm{H}), 7.57-7.43(\mathrm{~m}, 4 \mathrm{H}), 6.90(\mathrm{~d}, J=2.4 \mathrm{~Hz}, 2 \mathrm{H})$, $6.73(\mathrm{~d}, J=6.9 \mathrm{~Hz}, 1 \mathrm{H}), 6.58-6.57(\mathrm{~m}, 1 \mathrm{H}), 5.70(\mathrm{~A}$ of $\mathrm{AB}$, $\left.J_{A B}=12.6 \mathrm{~Hz}, 1 \mathrm{H}\right), 5.63\left(\mathrm{~B}\right.$ of $\left.\mathrm{AB}, J_{A B}=12.6 \mathrm{~Hz}, 1 \mathrm{H}\right), 4.87-$ $4.77(\mathrm{~m}, 1 \mathrm{H}), 3.80(\mathrm{~s}, 6 \mathrm{H}), 1.48(\mathrm{~d}, J=7.2 \mathrm{~Hz}, 3 \mathrm{H}) ;{ }^{13} \mathrm{C}$ NMR $\left(75 \mathrm{MHz}, \mathrm{CDCl}_{3}\right): \delta 173.20,166.92,161.11,136.36$, $133.98,131.79,130.98,129.84,129.00,127.91,126.97$, $126.28,125.45,123.62,105.16,104.17,66.00,55.79$, 48.99, 18.76; IR (KBr, cm $\left.{ }^{-1}\right) 3319,2925,1741,1645,1594,1532,1456,1354,1205,1156,1063$, 776; MS: HR-ESI calculated for $\mathrm{C}_{23} \mathrm{H}_{37} \mathrm{NO}_{5} \mathrm{H}\left(\mathrm{M}+\mathrm{H}^{+}\right) \mathrm{m} / \mathrm{z}$ : 394.1649, found $\mathrm{m} / \mathrm{z}$ : 394.1640. HPLC (CHIRALCEL OD-H, IPA/hexane=3/10, $1.0 \mathrm{~mL} / \mathrm{min}$ ): (S)-enantiomer: $33.4 \mathrm{~min}$; (R)enantiomer: $38.6 \mathrm{~min}$.<smiles>COc1cc(OC)cc(C(=O)NC(C(=O)OCc2cccc3ccccc23)C(C)C)c1</smiles>

${ }^{1}$ HNMR $\left(300 \mathrm{MHz}, \mathrm{CDCl}_{3}\right): \delta 8.03-7.99(\mathrm{~m}, 1 \mathrm{H}), 7.91-$ $7.86(\mathrm{~m}, 2 \mathrm{H}), 7.58-7.43(\mathrm{~m}, 4 \mathrm{H}), 6.90(\mathrm{~d}, J=2.1 \mathrm{~Hz}, 2 \mathrm{H})$, 6.60-6.58 (m, 2H), $5.70\left(\mathrm{~A}\right.$ of $\left.\mathrm{AB}, J_{A B}=12.0 \mathrm{~Hz}, 1 \mathrm{H}\right), 5.62$ $\left(\mathrm{B}\right.$ of $\left.\mathrm{AB}, J_{A B}=12.0 \mathrm{~Hz}, 1 \mathrm{H}\right), 4.82\left(\mathrm{dd}, J_{1}=8.7 \mathrm{~Hz}, J_{2}=\right.$ $4.5 \mathrm{~Hz}, 1 \mathrm{H}), 3.80(\mathrm{~s}, 6 \mathrm{H}), 2.32-2.17(\mathrm{~m}, 1 \mathrm{H}), 0.94(\mathrm{~d}, J=$ $6.6 \mathrm{~Hz}, 3 \mathrm{H}), 0.87(\mathrm{~d}, \quad J=6.9 \mathrm{~Hz}, 3 \mathrm{H}) ;{ }^{13} \mathbf{C}$ NMR $(75$ $\left.\mathrm{MHz}, \mathrm{CDCl}_{3}\right): \delta 172.18,167.40,161.13,136.64,133.95$, $131.80,130.99,129.84,128.99,128.11,126.94,126.27$, $125.45,123.65,105.23,103.96,65.73,57.76,55.79,31.84,19.27,17.91 ; \quad$ IR $\left(\mathrm{KBr}, \mathrm{cm}^{-1}\right) 3328$, 2961, 2924, 1736, 1654, 1594, 1525, 1458, 1355, 1205, 1156, 1064, 794, 777. MS: HR-ESI calculated for $\mathrm{C}_{25} \mathrm{H}_{27} \mathrm{NO}_{5} \mathrm{H}\left(\mathrm{M}+\mathrm{H}^{+}\right) \mathrm{m} / \mathrm{z}$ : 422.1962, found $\mathrm{m} / \mathrm{z}$ : 422.1960. HPLC (for alcohol obtained by LAH reduction) (CHIRALCEL AS-H, IPA/hexane=1/10, $1.0 \mathrm{~mL} / \mathrm{min}$ ): (S)enantiomer: $13.2 \mathrm{~min}$; (R)-enantiomer: $26.7 \mathrm{~min}$. 


\section{References.}

1. (a) Zhu, S. S.; Cefalo, D. R.; La, D. S.; Jamieson, J. Y.; Davis, W. M.; Hoveyda, A. H.; Schrock, R. R. J. Am. Chem. Soc. 1999, 121, 8251 (b) Wipf, P.; Jung, J.-K. J. Org. Chem. 2000, 65, 6319. (c) Wu, T. R.; Shen, L.; Chong, J. M.; Org. Lett. 2004, 6, 2701. (d) Akiyama, T.; Itoh, J.; Yokota, K.; Fuchibe, K. Angew. Chem., Int. Ed. 2004, 43, 1566. (e) Storer, R. I.; Carrera, D. E.; Ni, Y.; MacMillan, D. W. C.; J. Am. Chem. Soc. 2006, 128, 84. f) Singh, R.; Czekelius, C.; Schrock, R. R.; Mueller, P.; Hoveyda, A. H.; Organometallics, 2007, 26, 2528.

2. Liang, J.; Ruble, J. C.; Fu, G. C. J. Org. Chem. 1998, 63, 3154.

3. Park, Y.-L.; Son, J.-H.; Kang, J.-S.; Kim, S.-K.; Lee, J.-H.; Park, J.-W. Chem. Commun. 2008, 2143. 

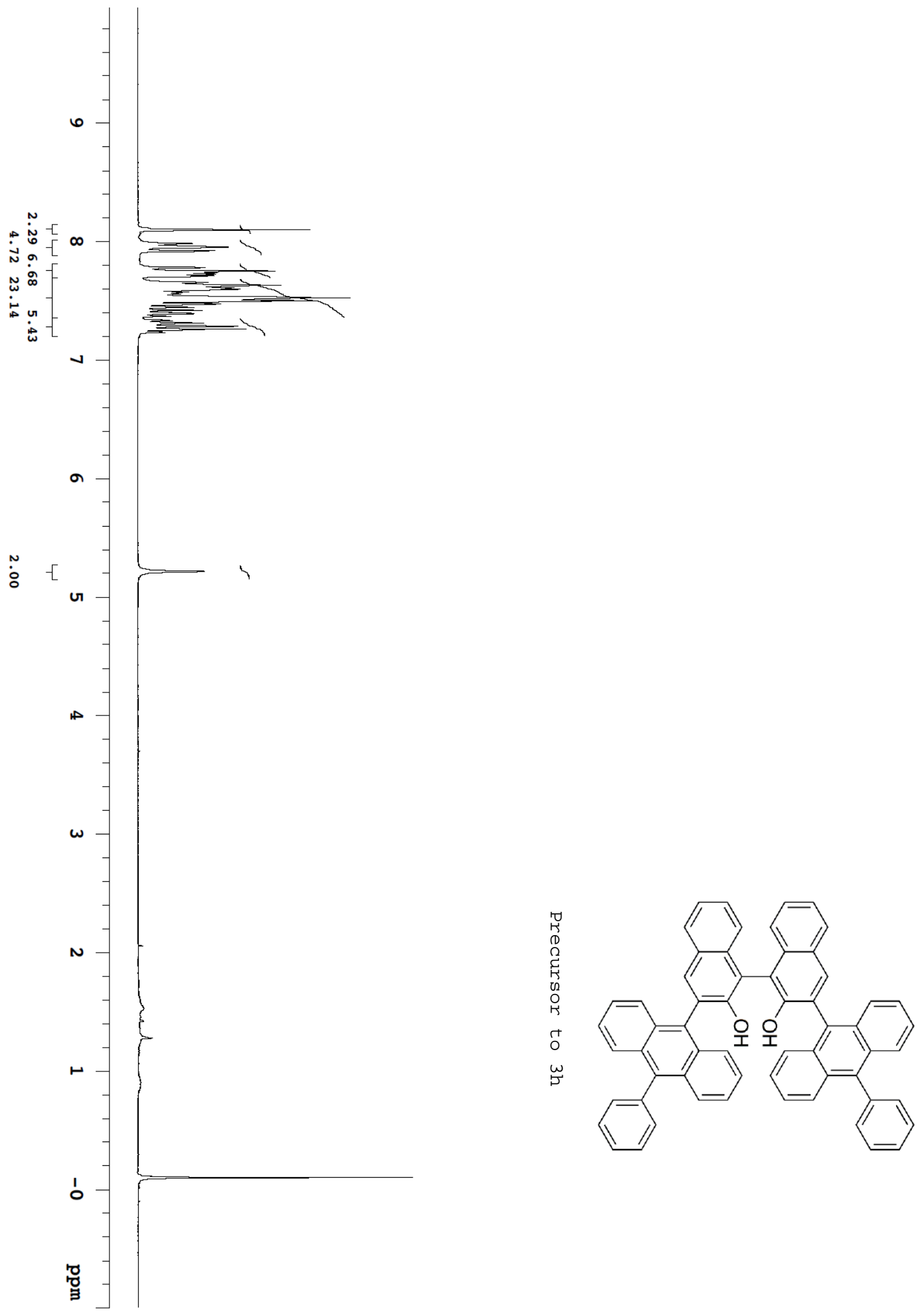


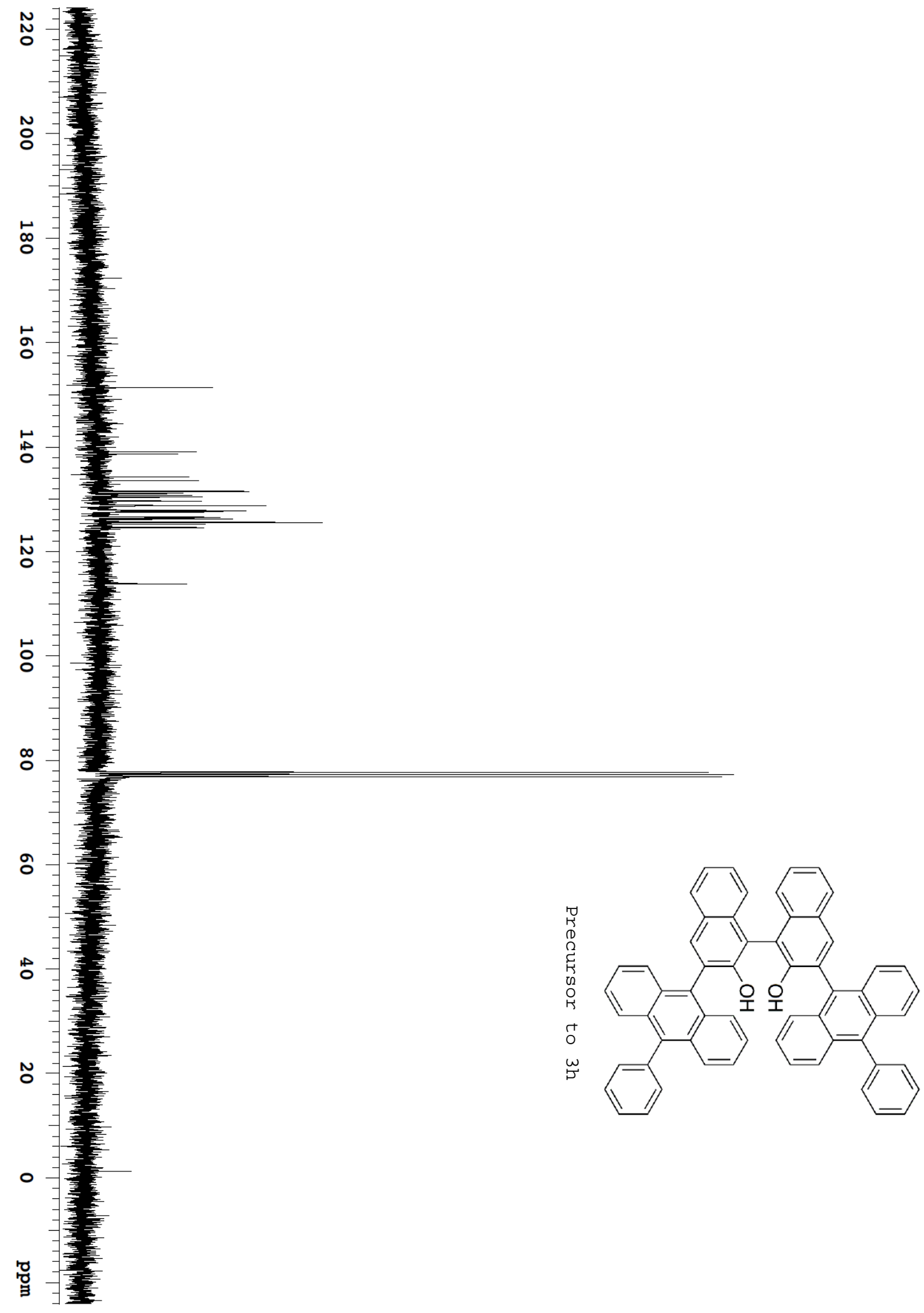




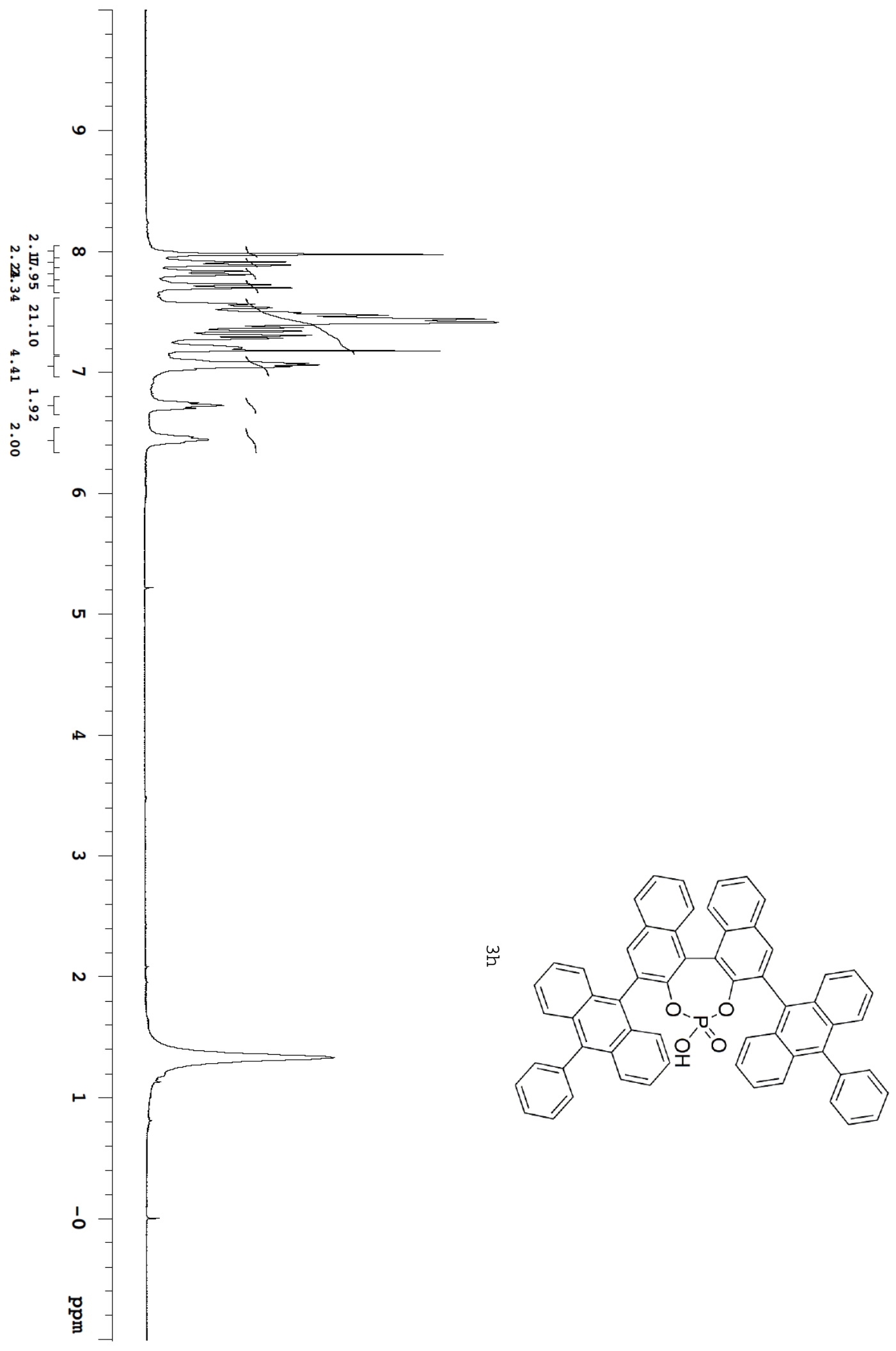




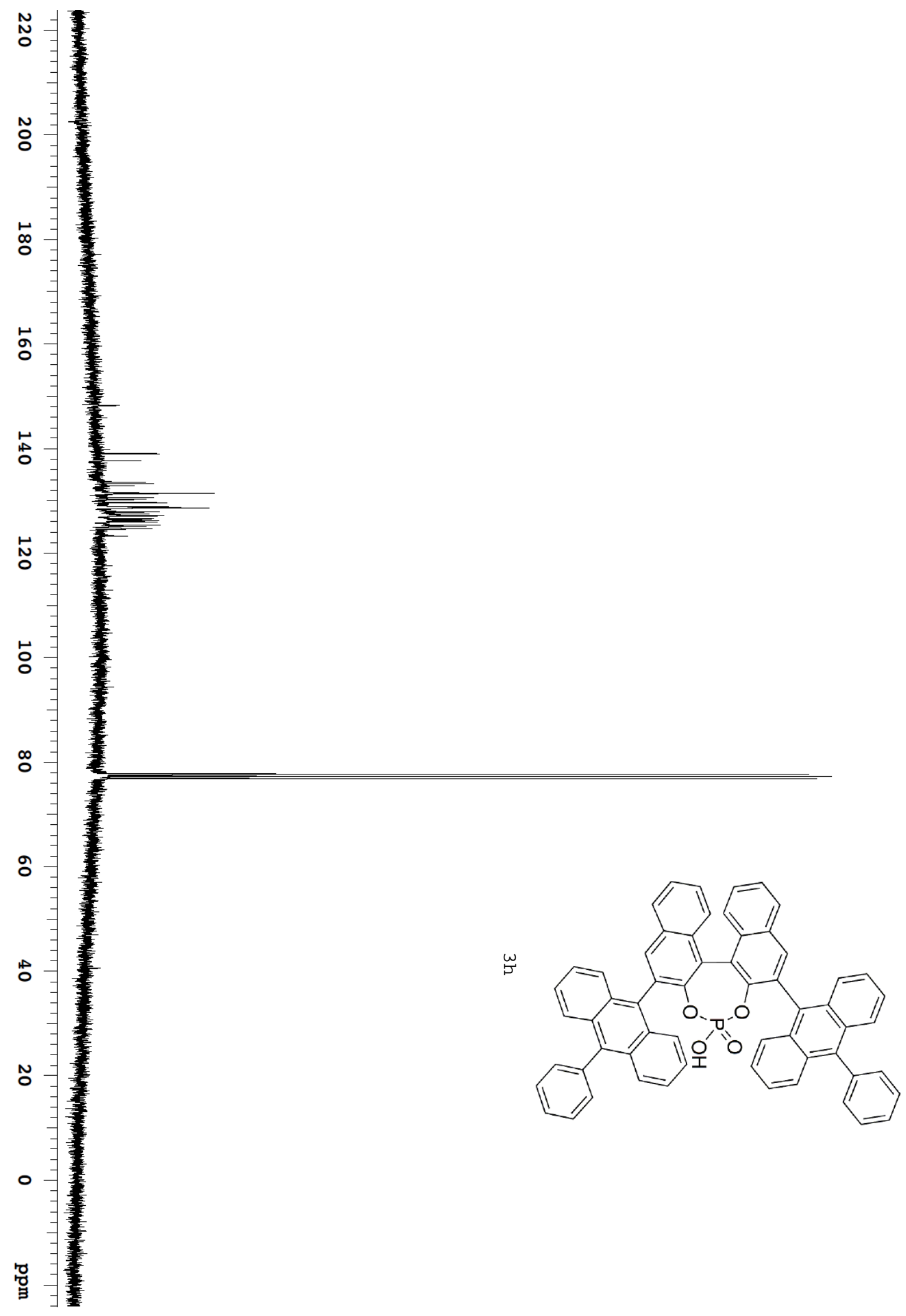



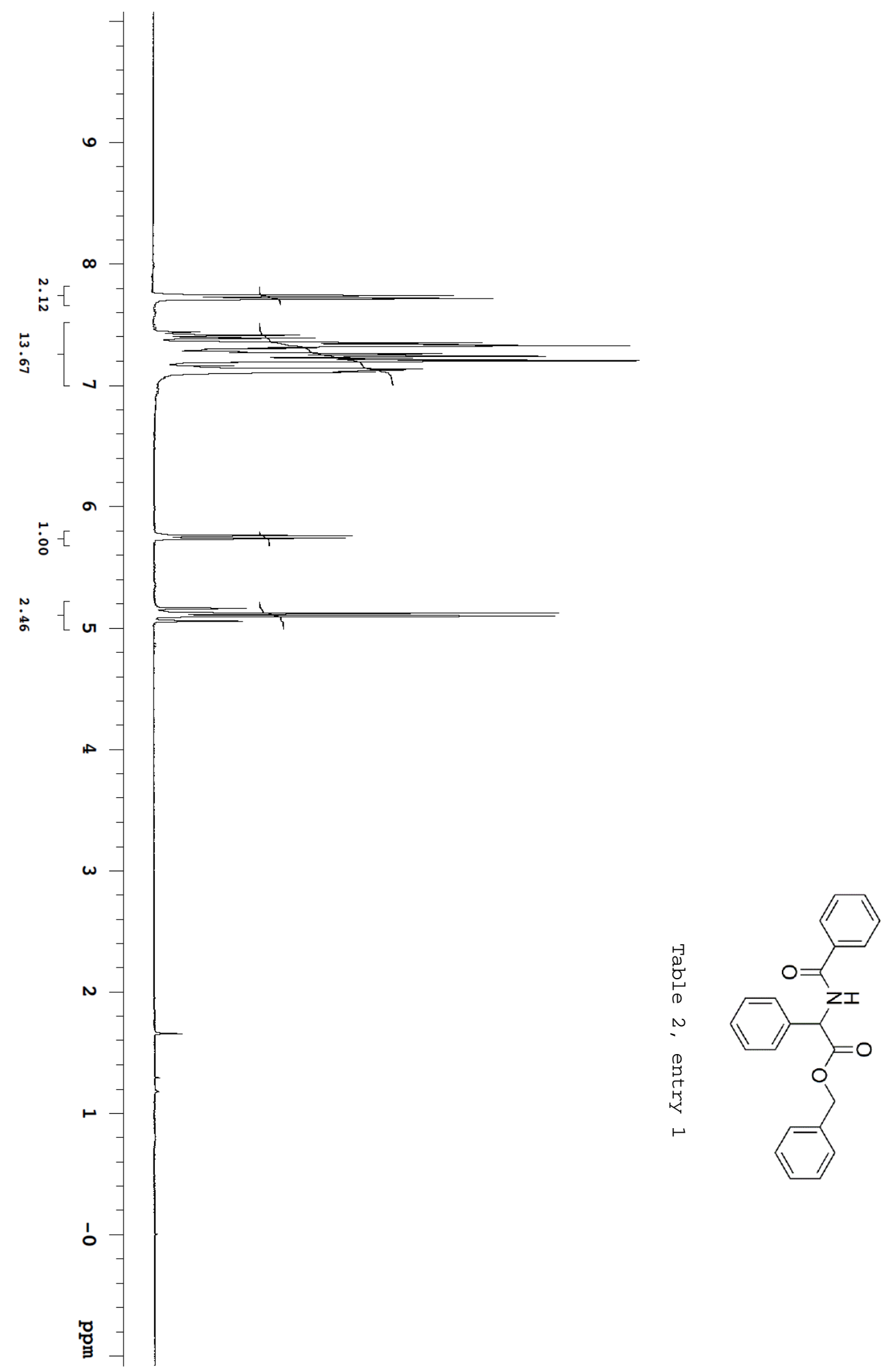

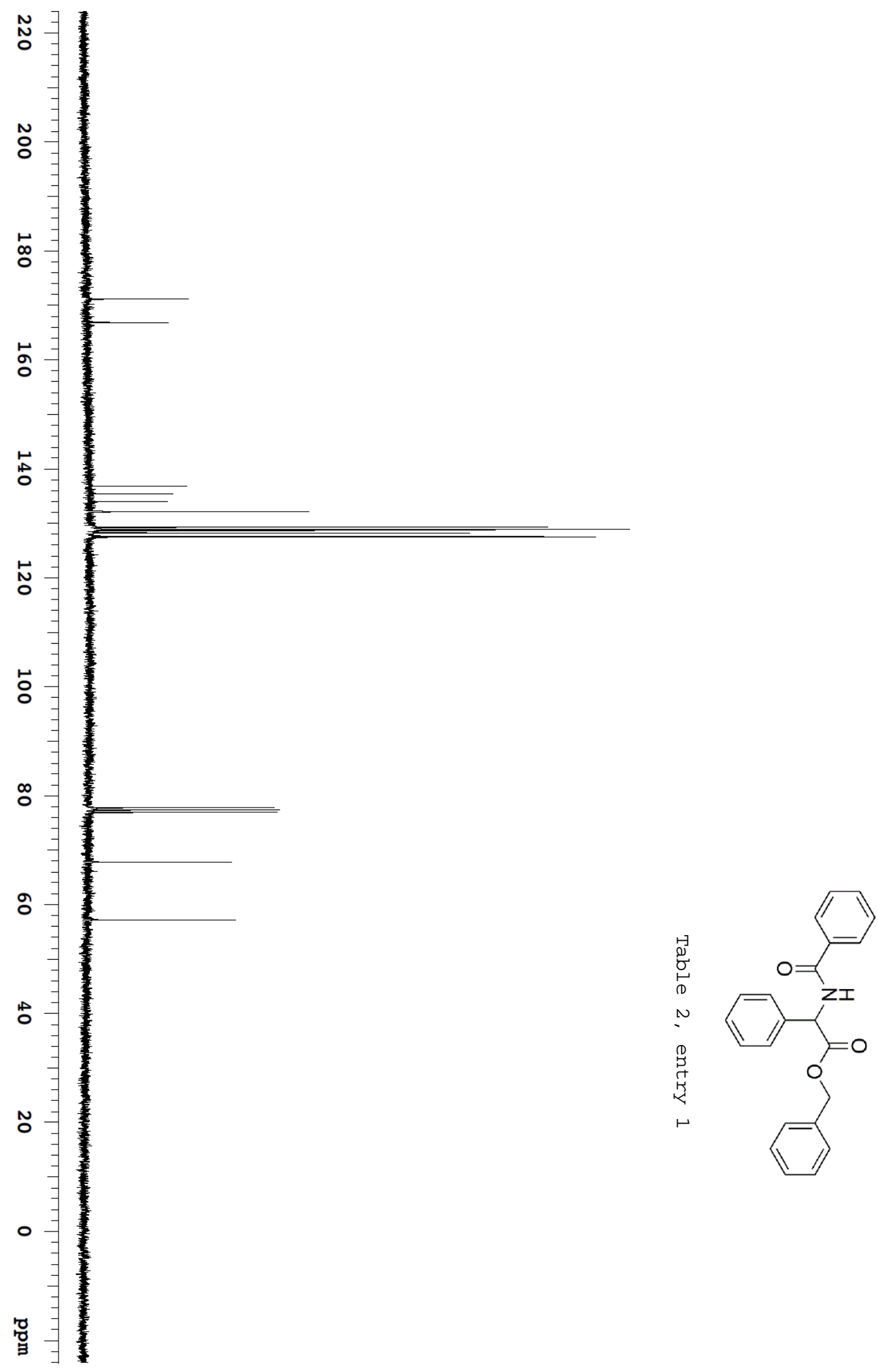

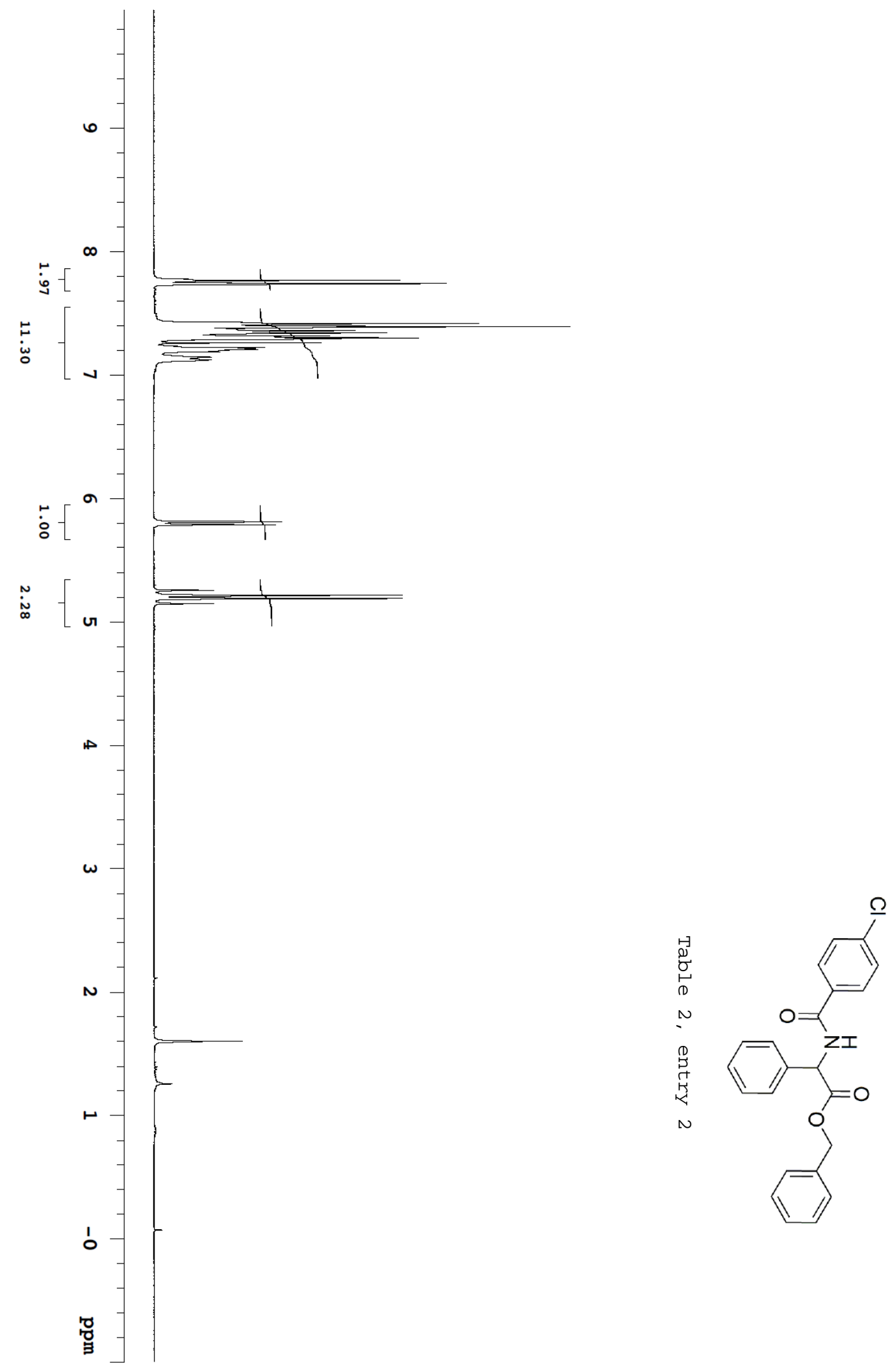


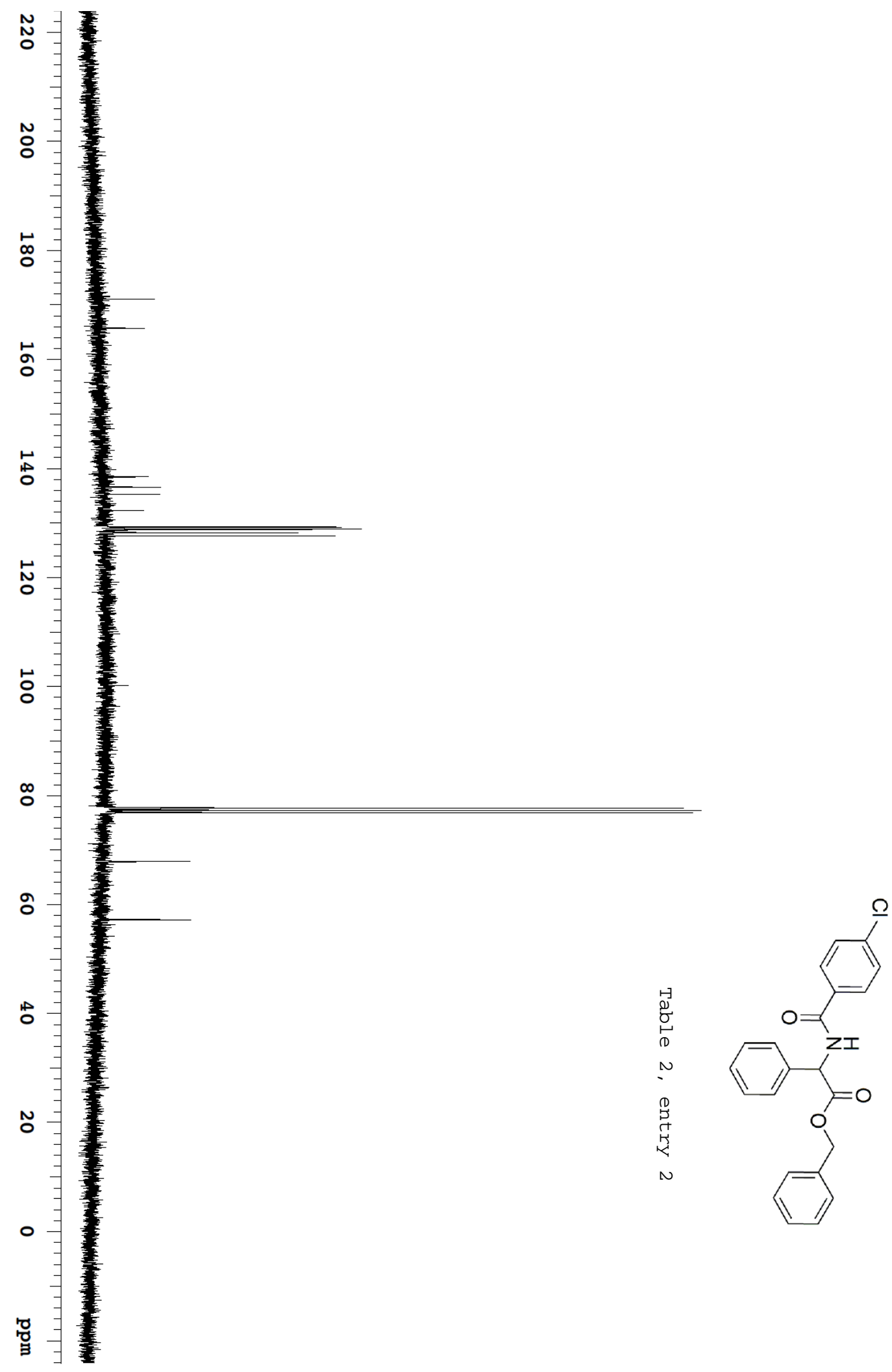




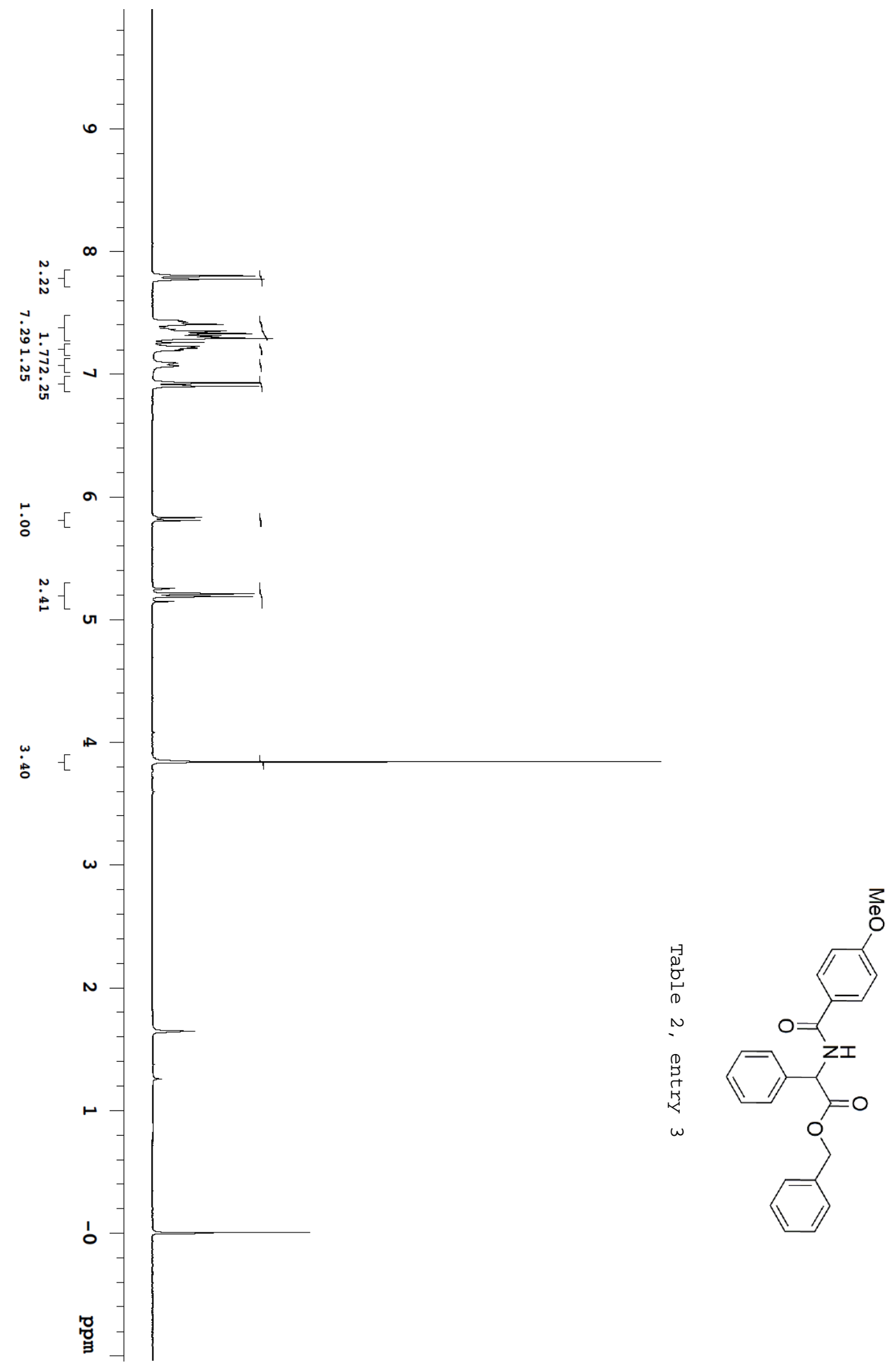




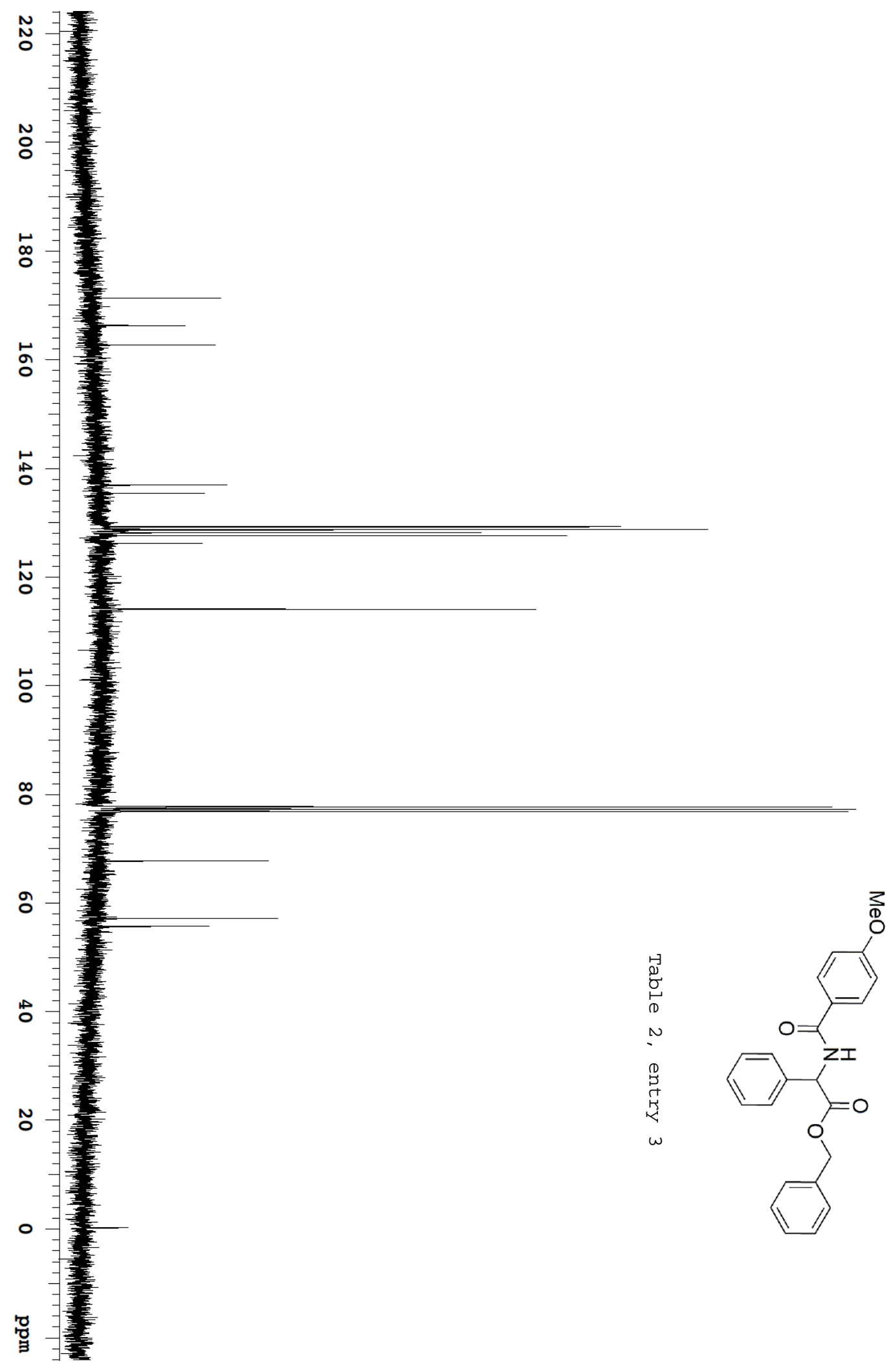




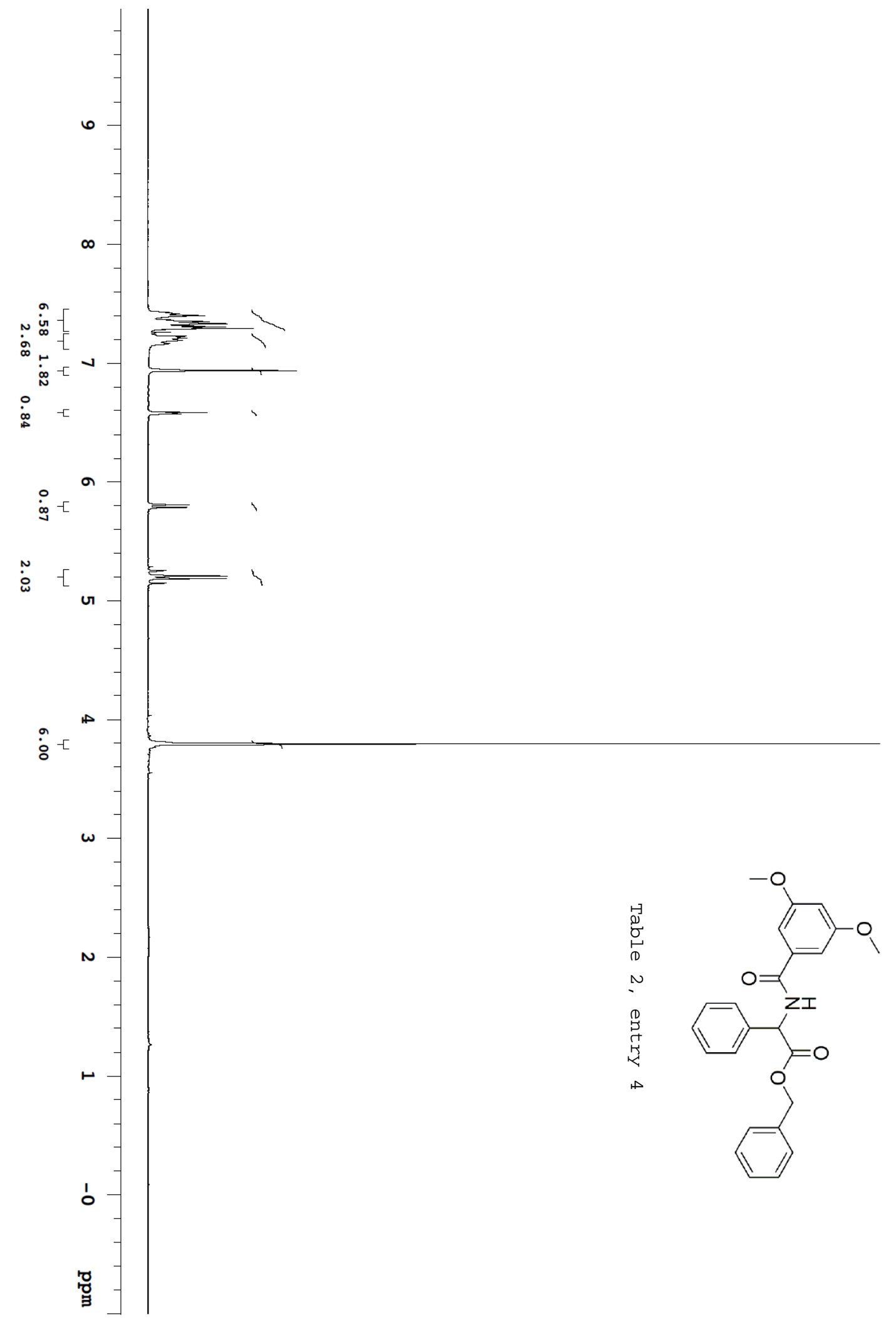



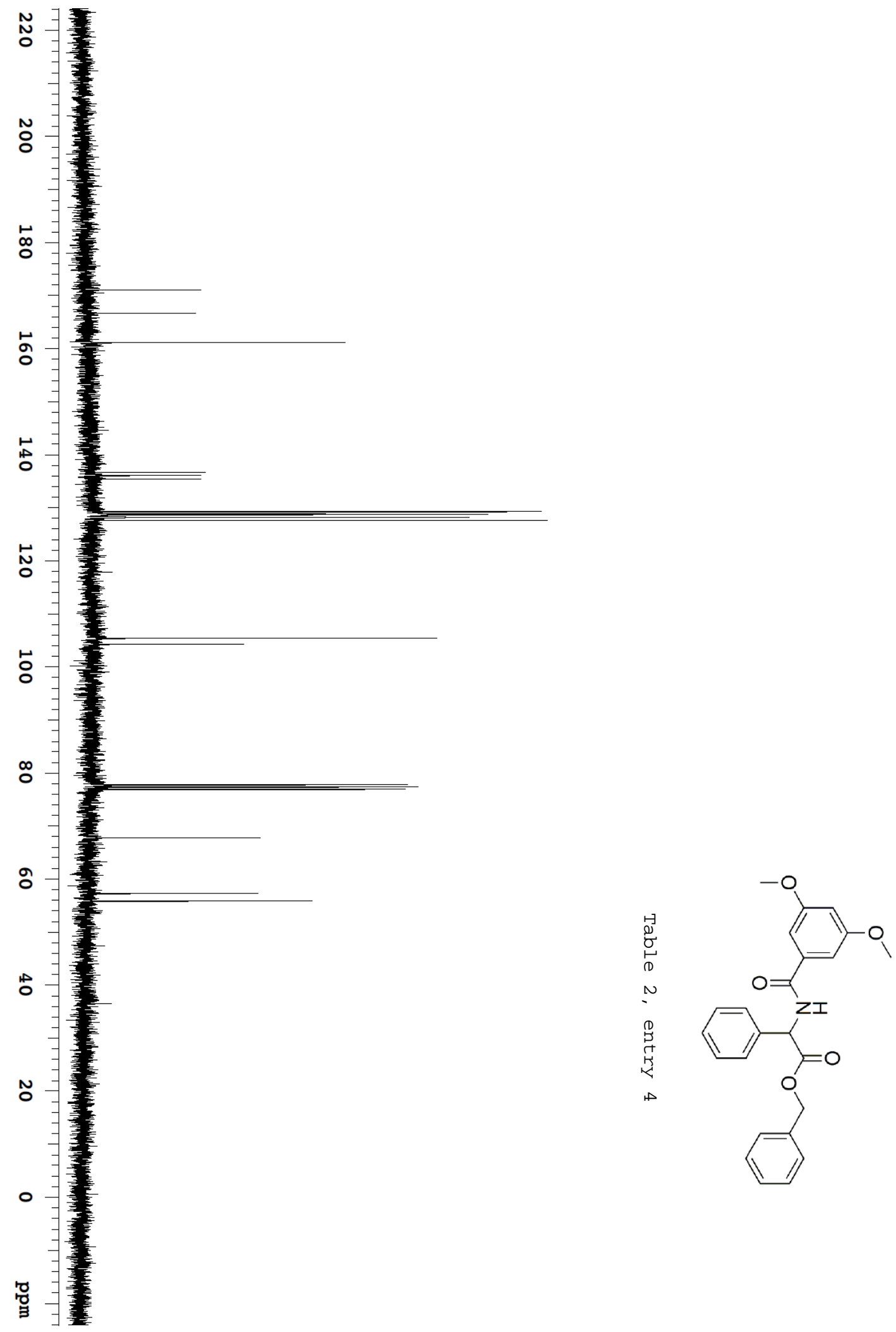


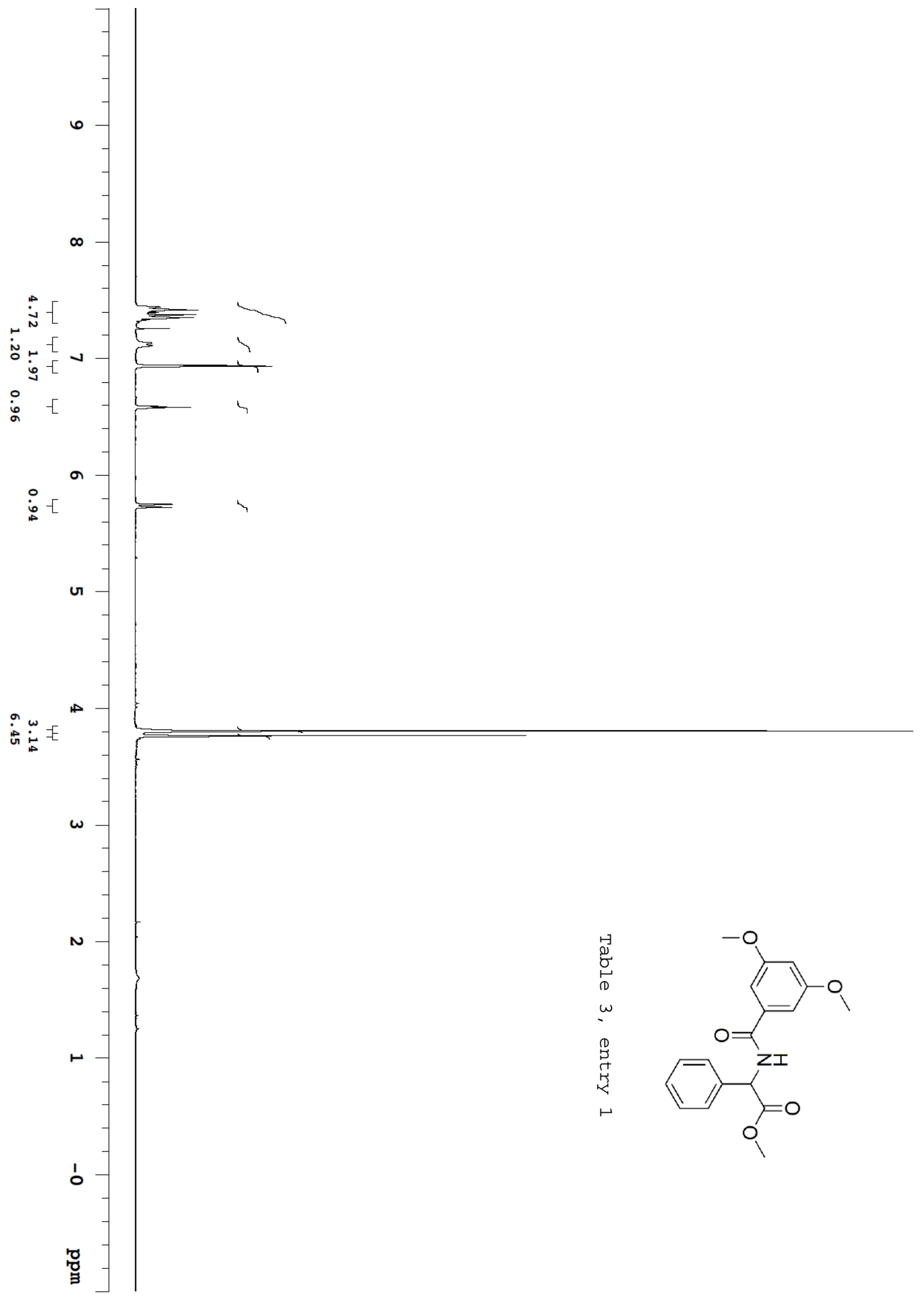



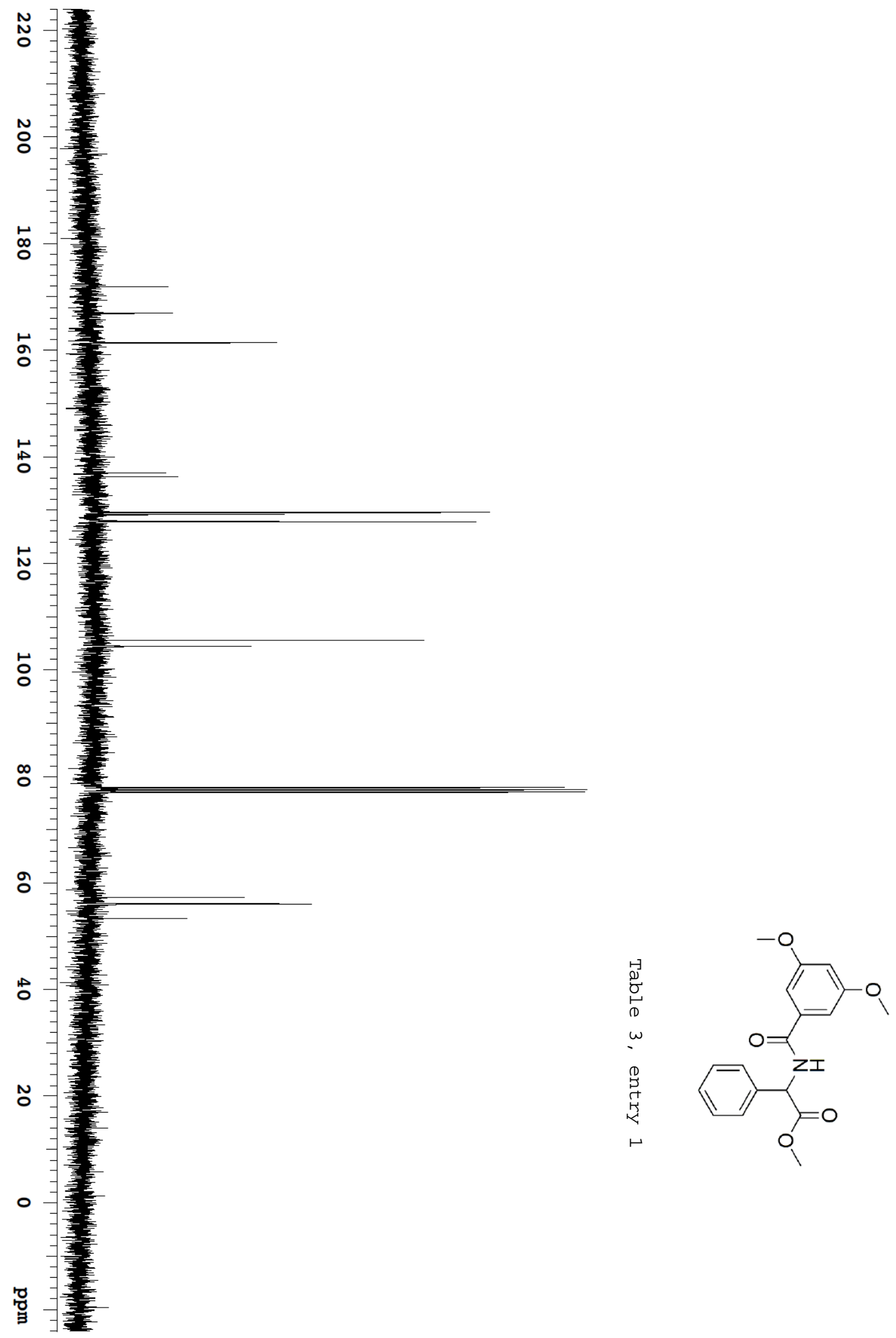


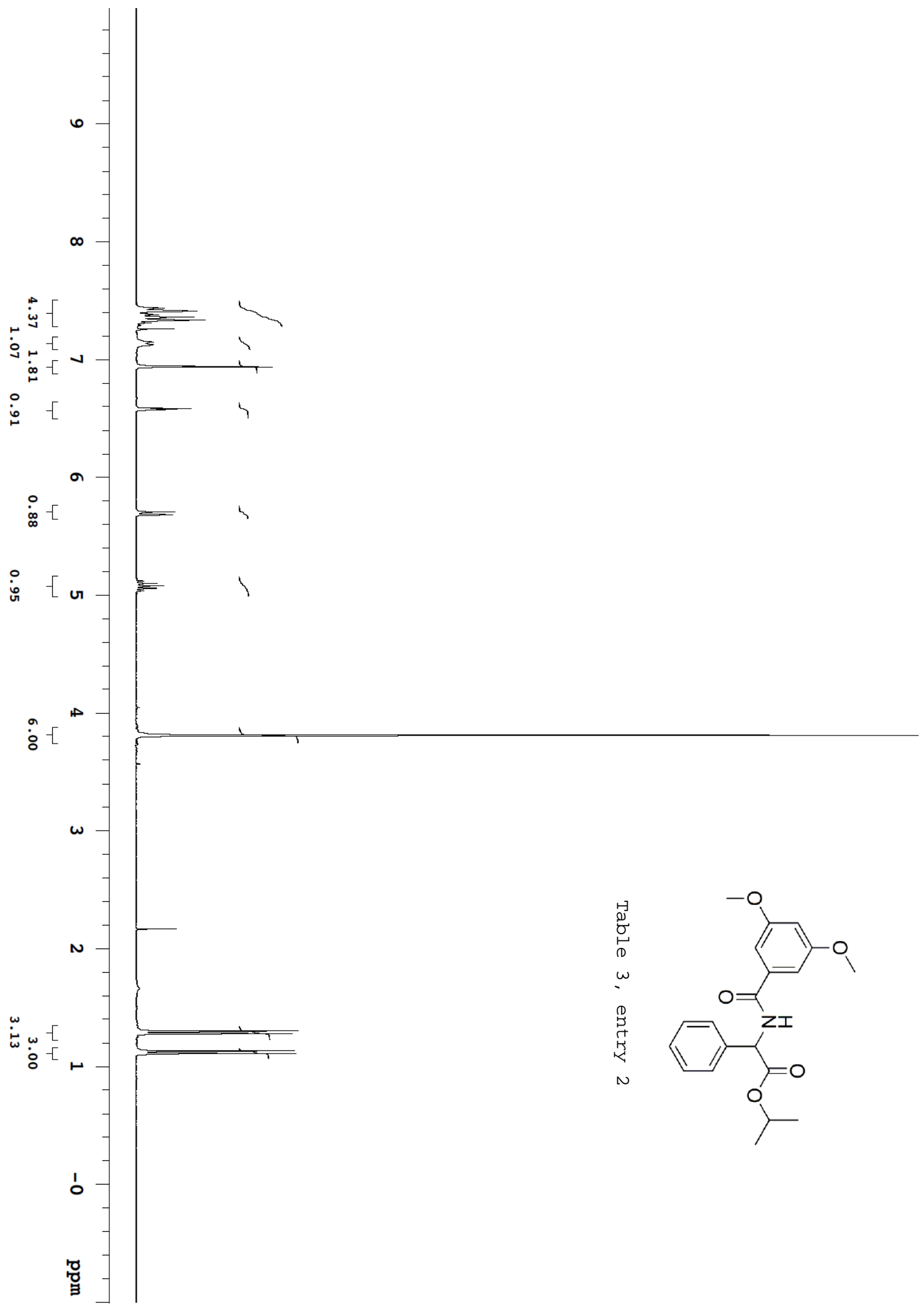



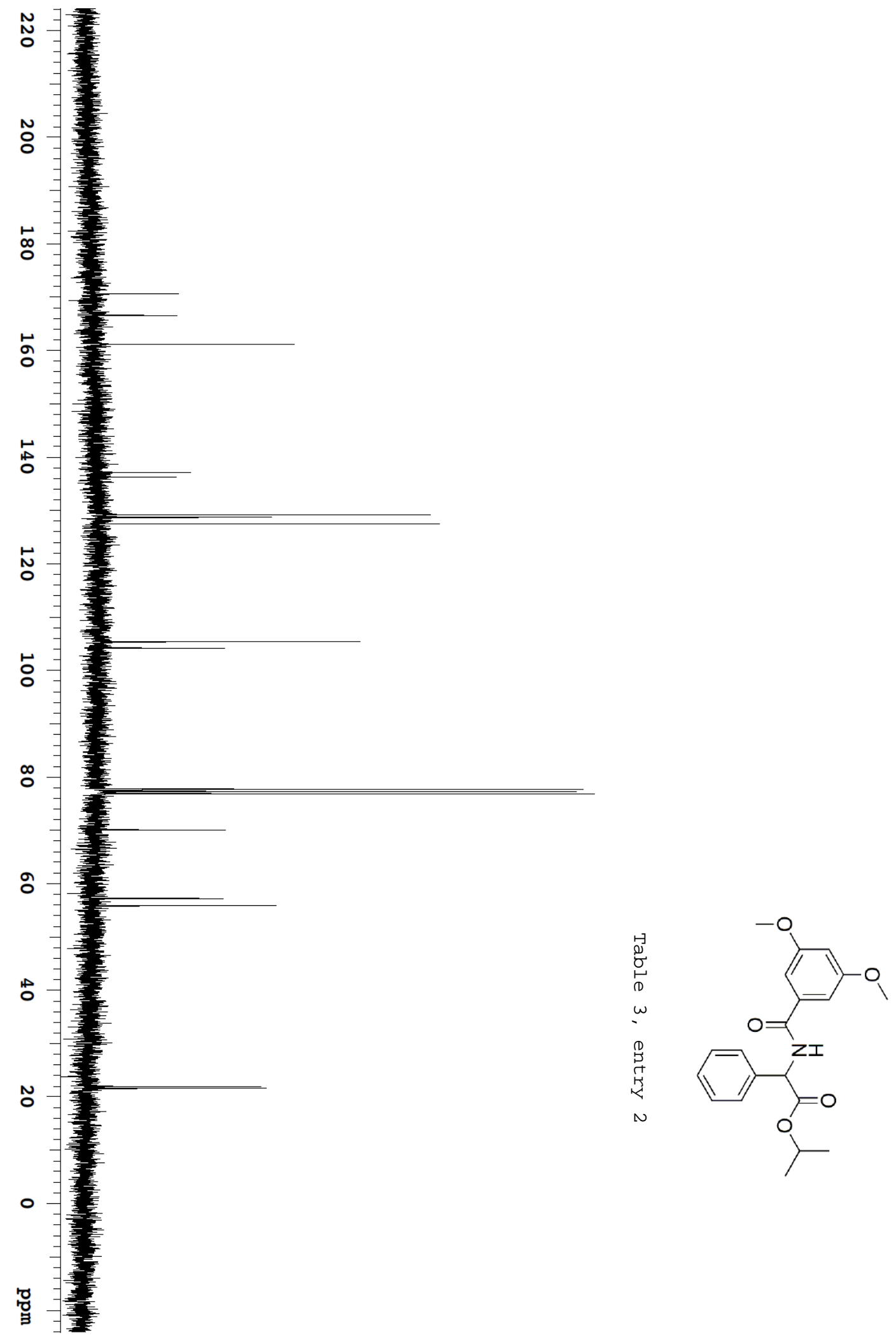


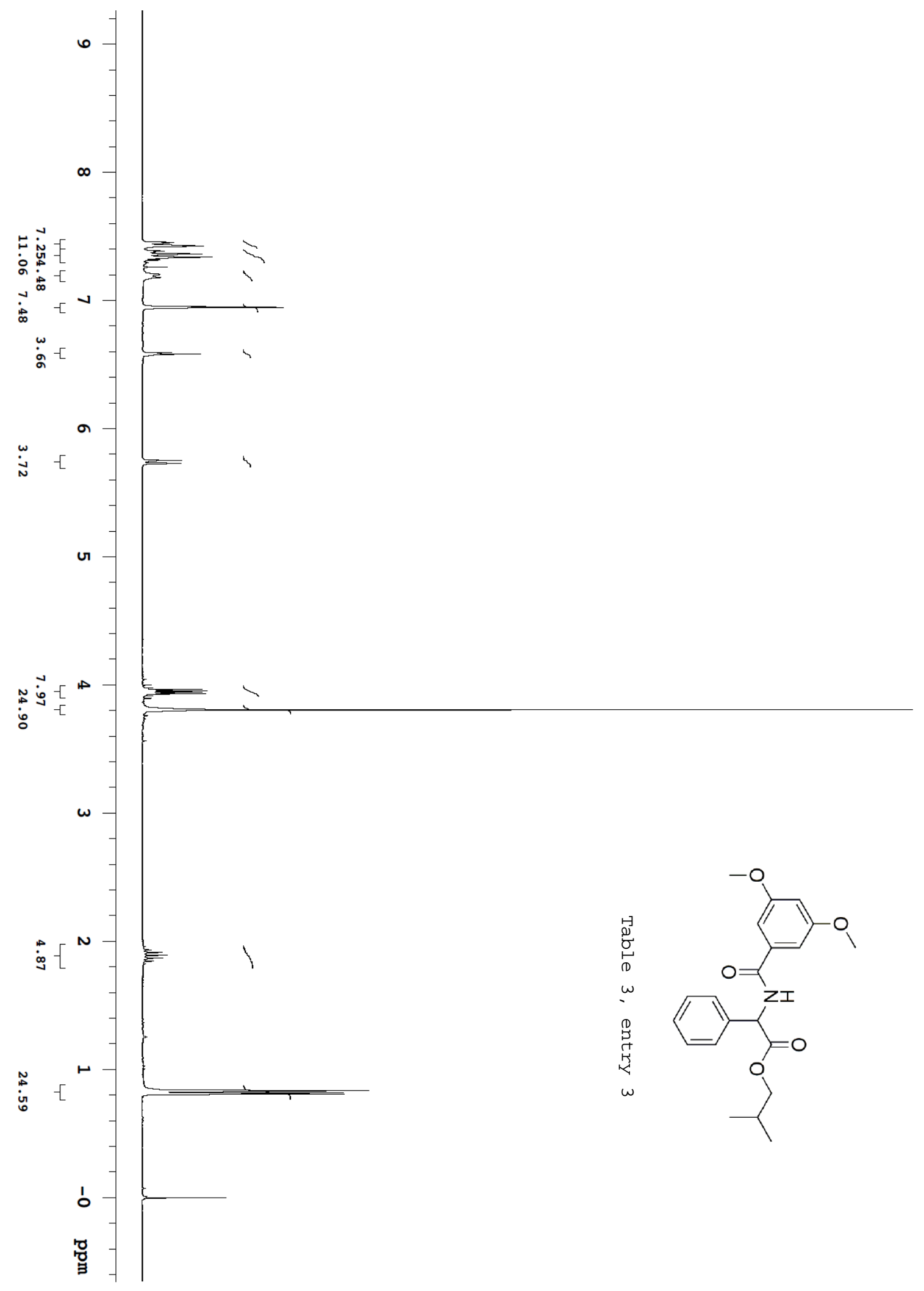



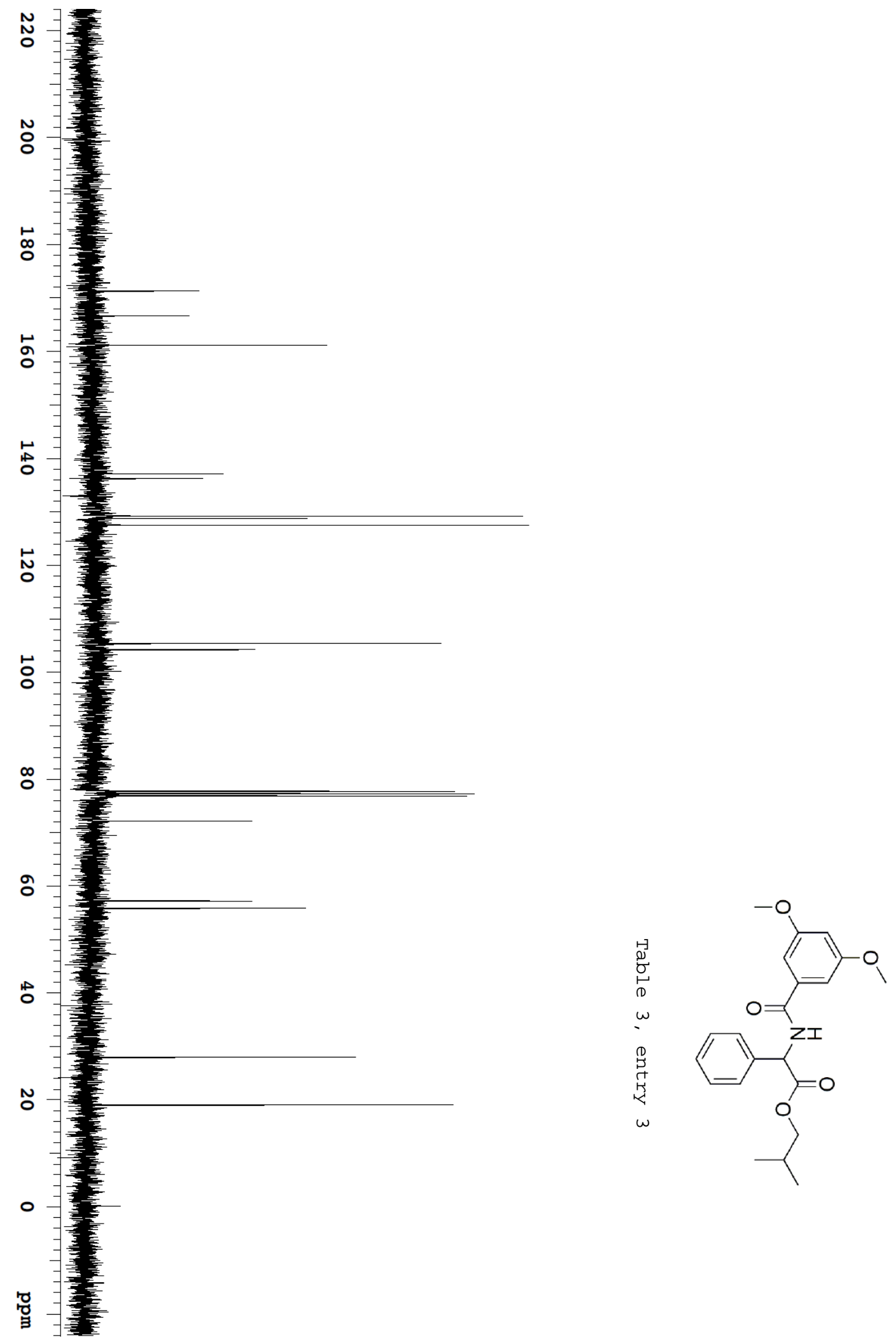


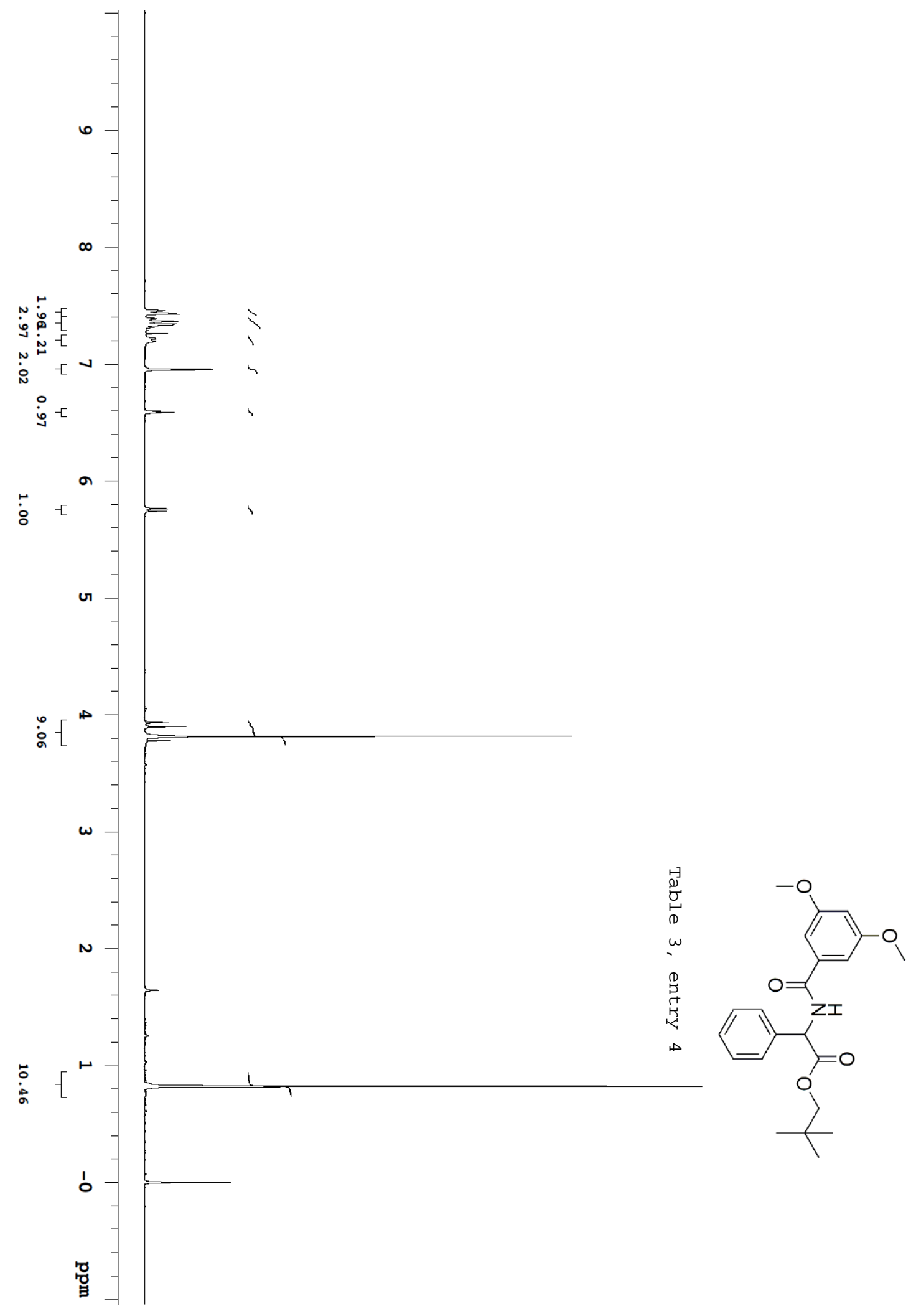




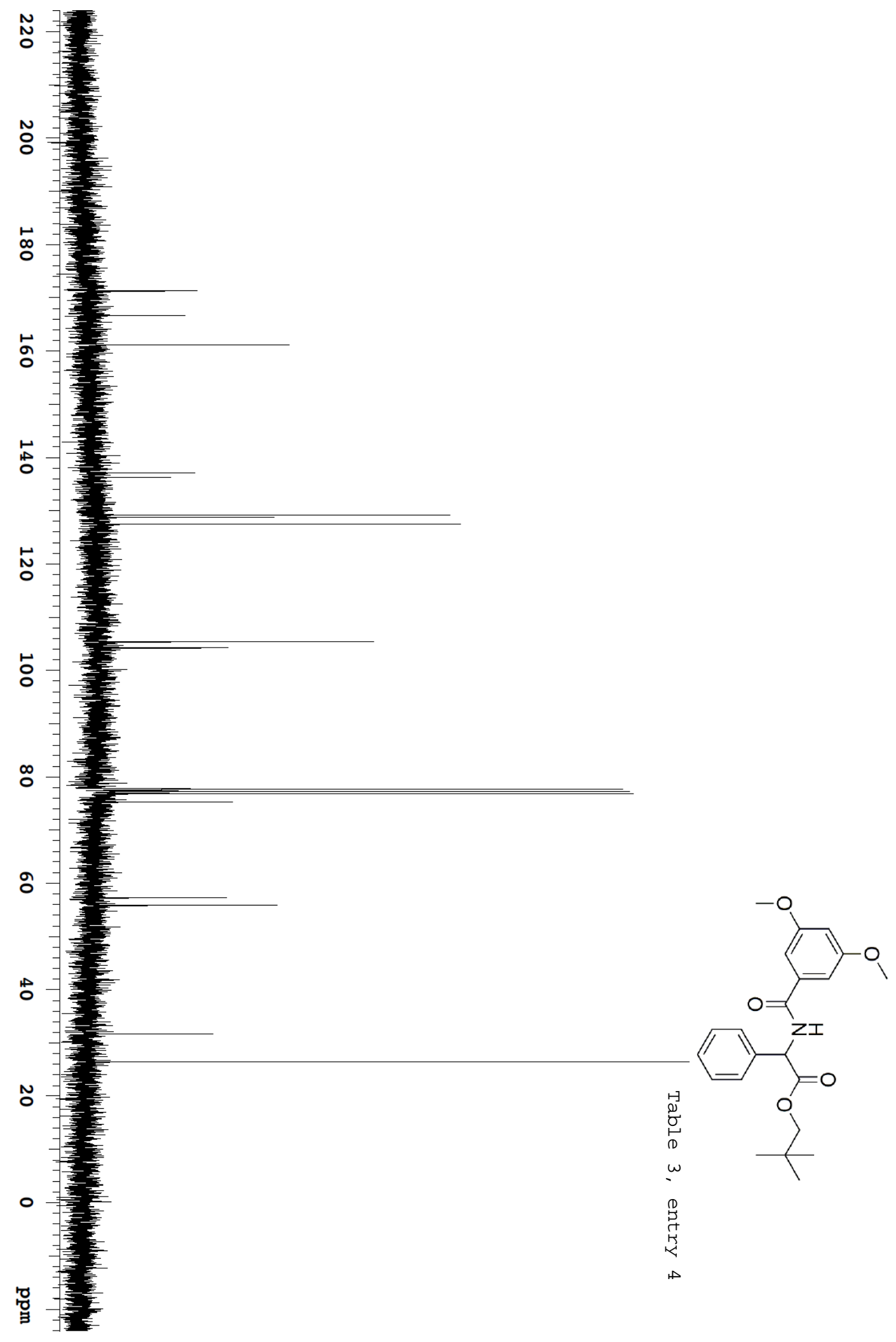




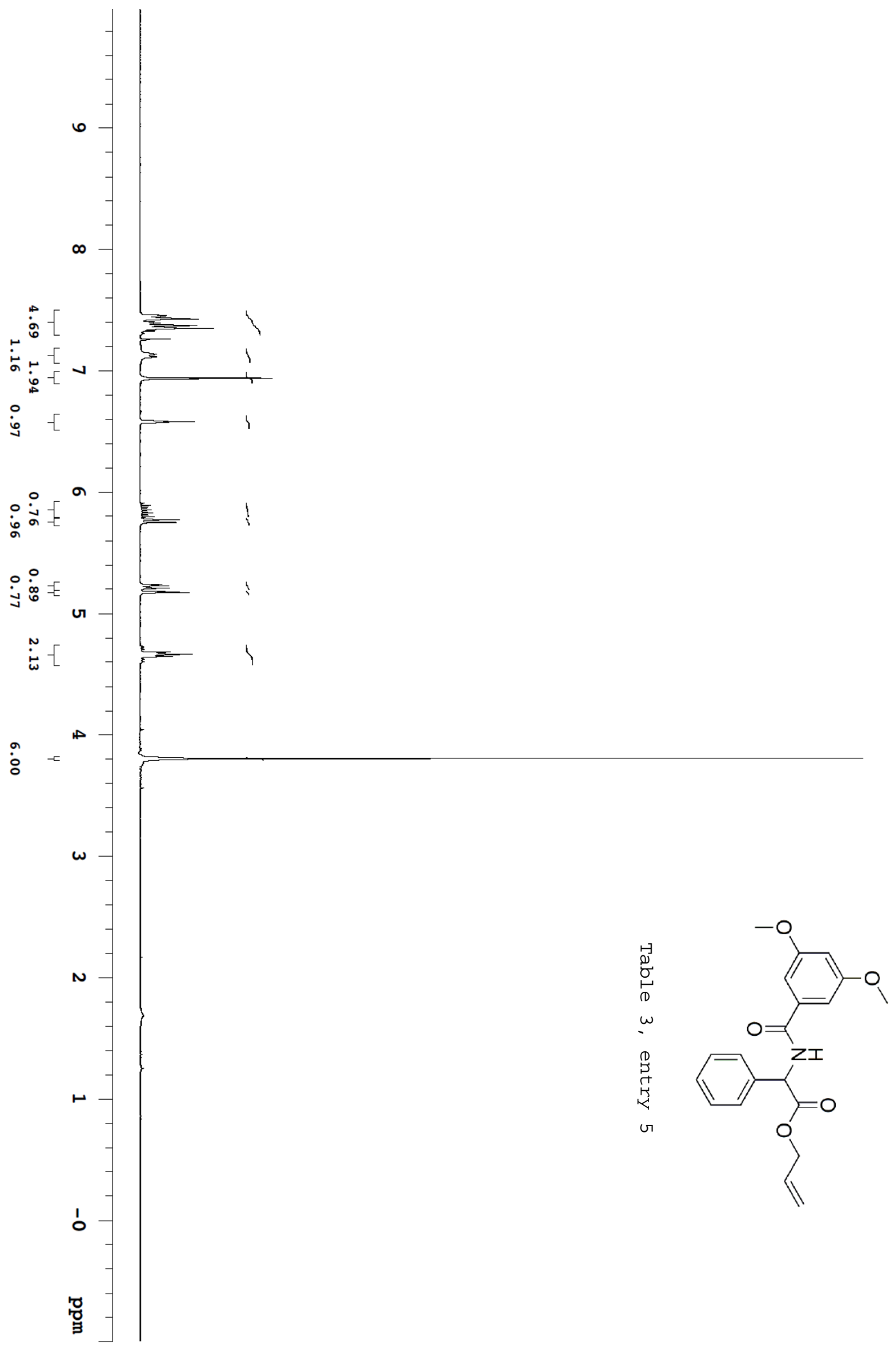



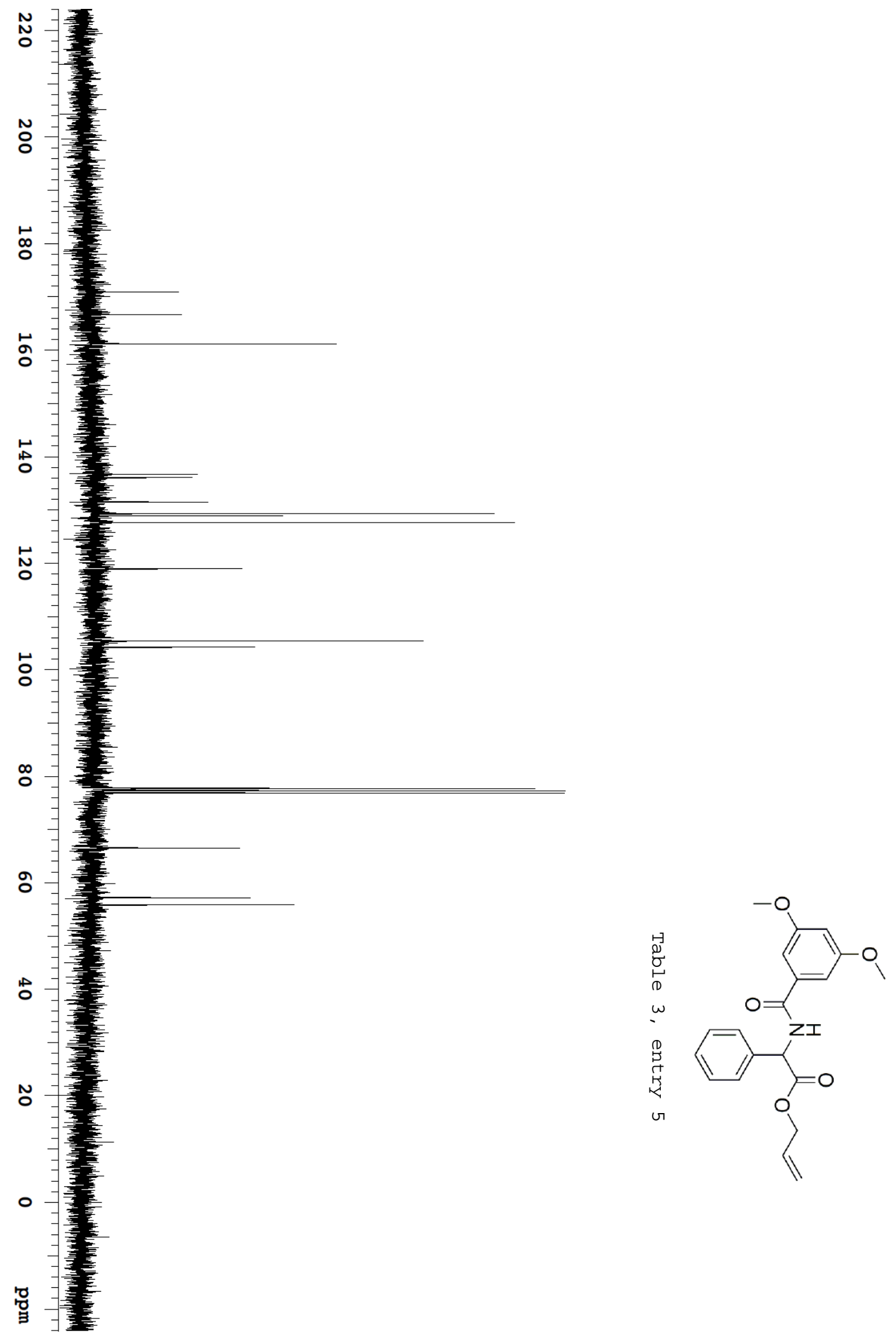


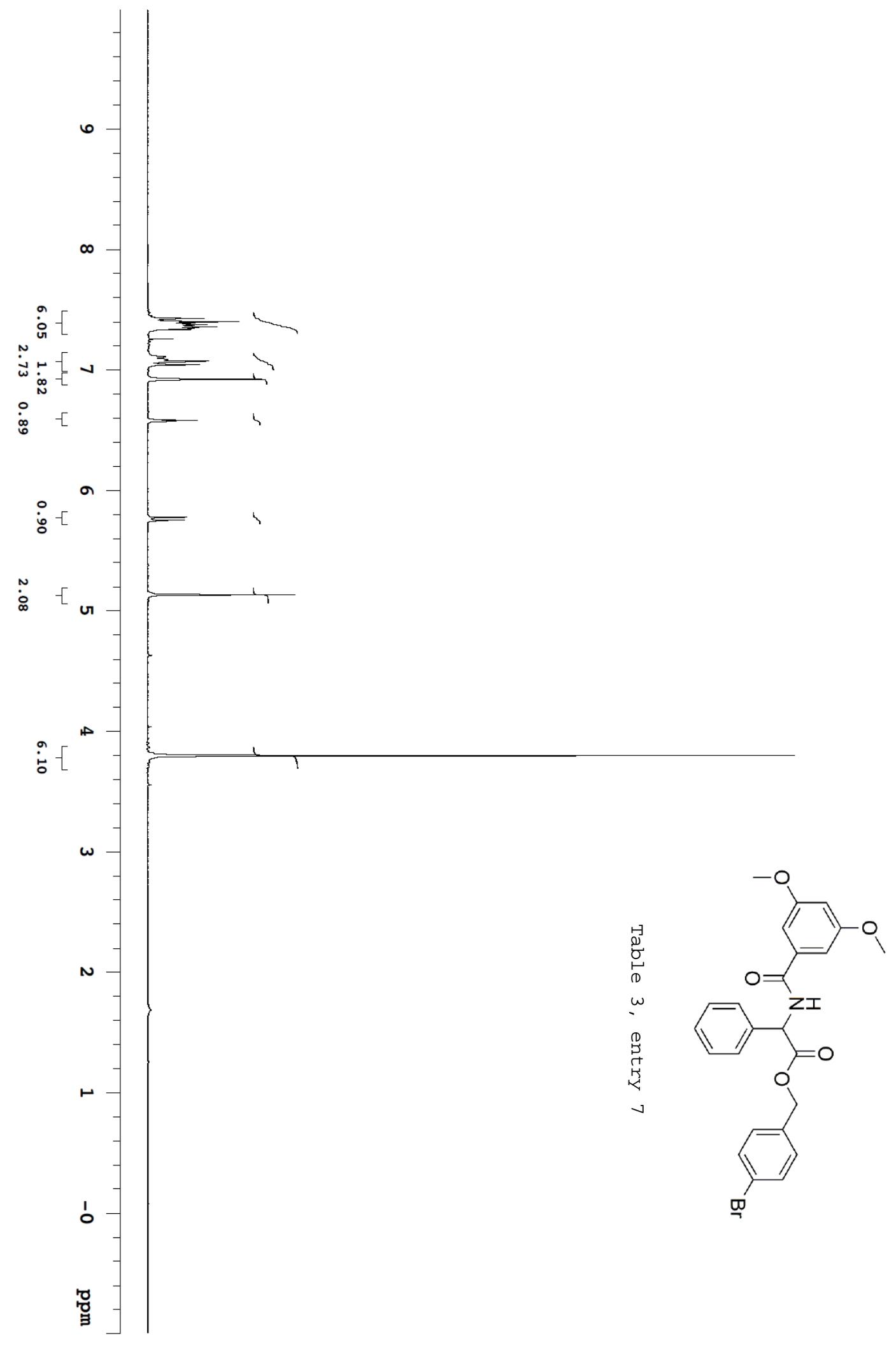




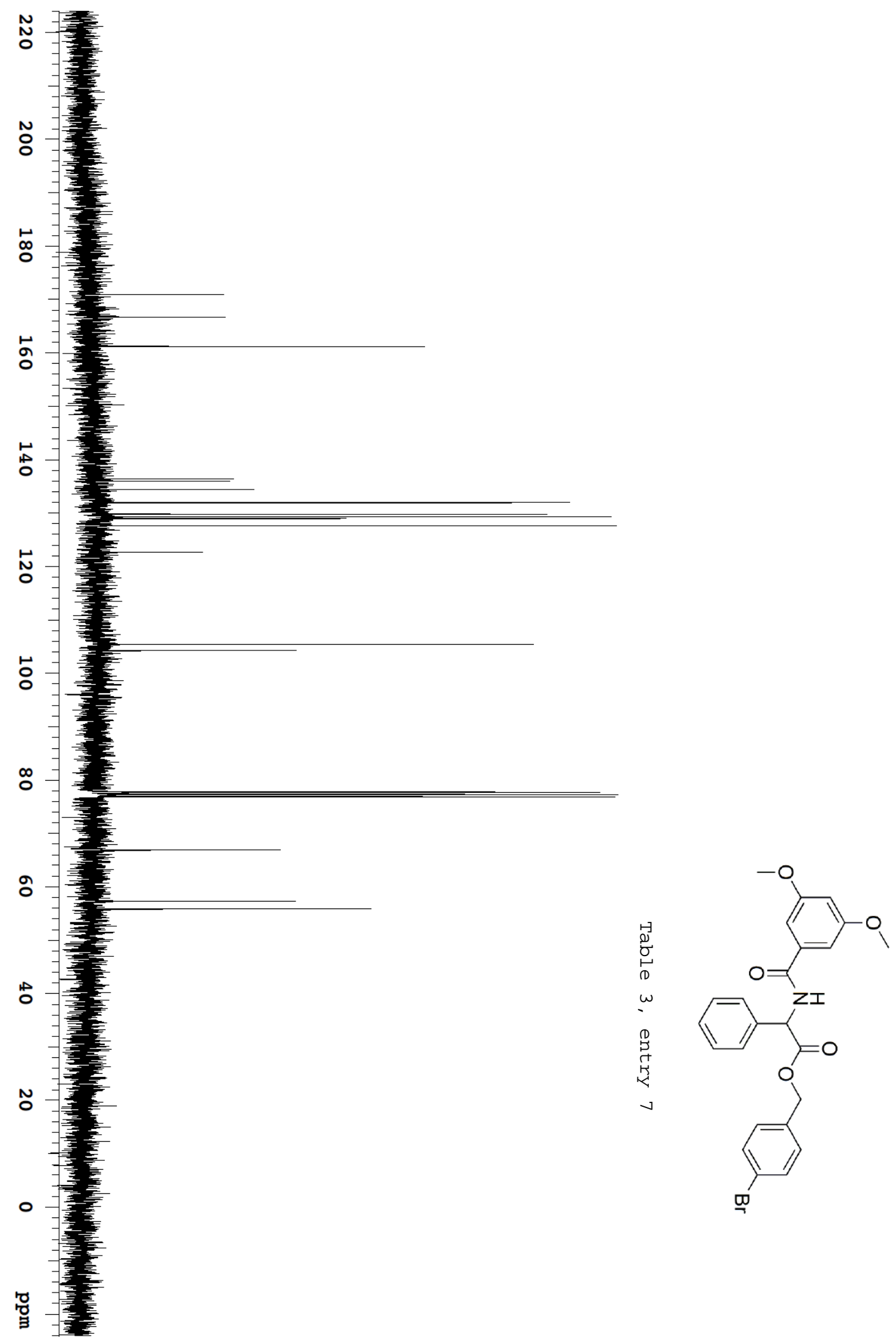




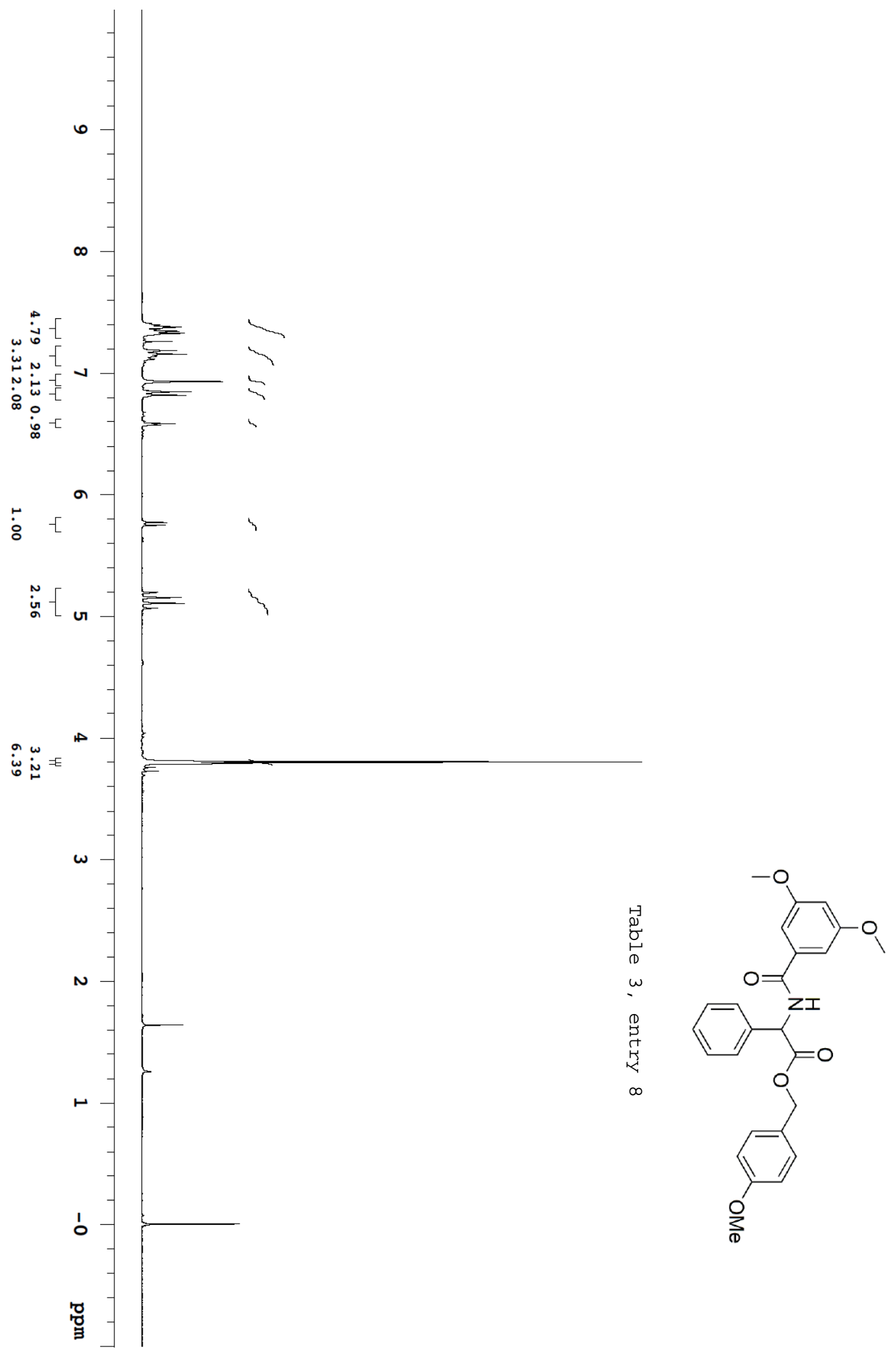




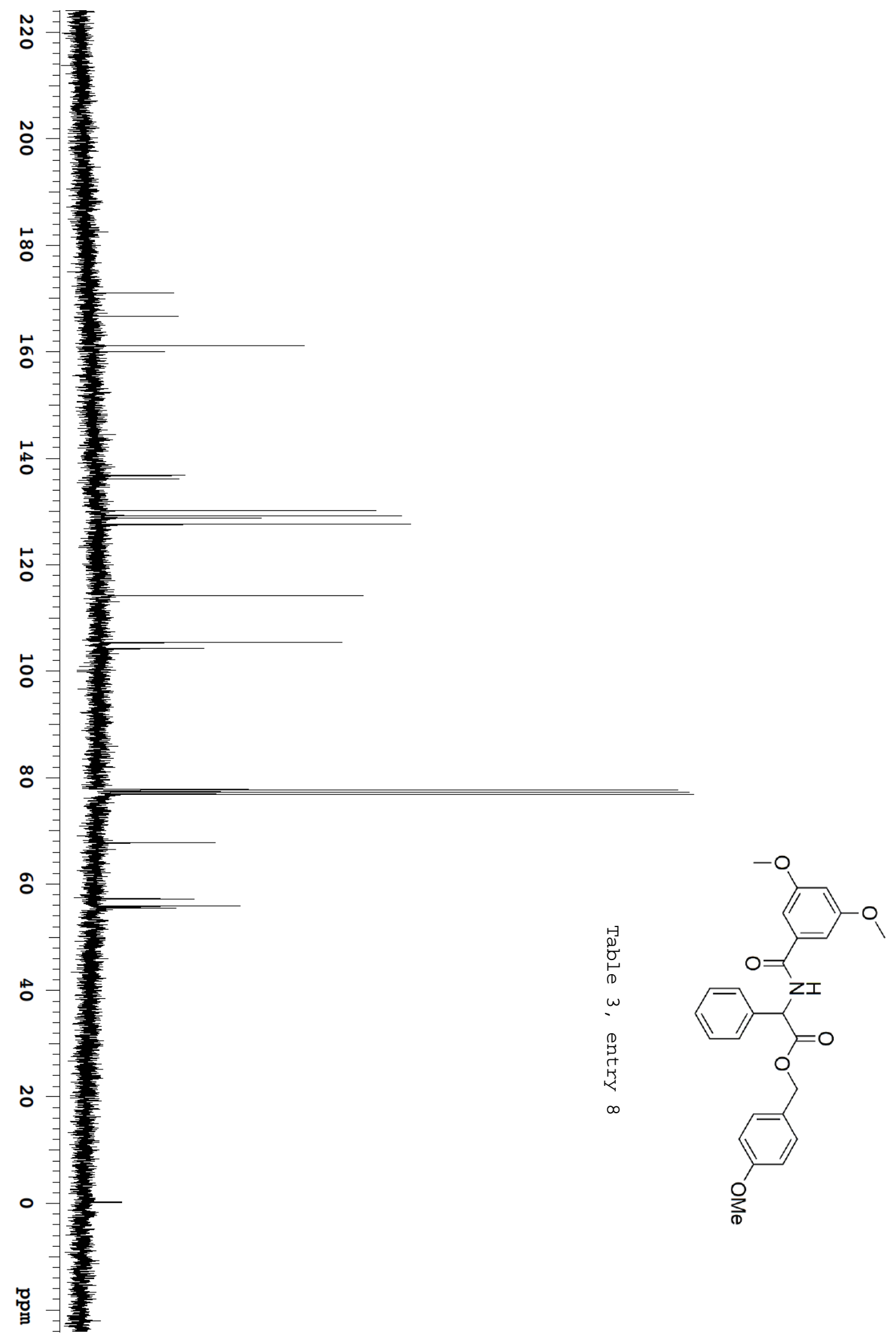




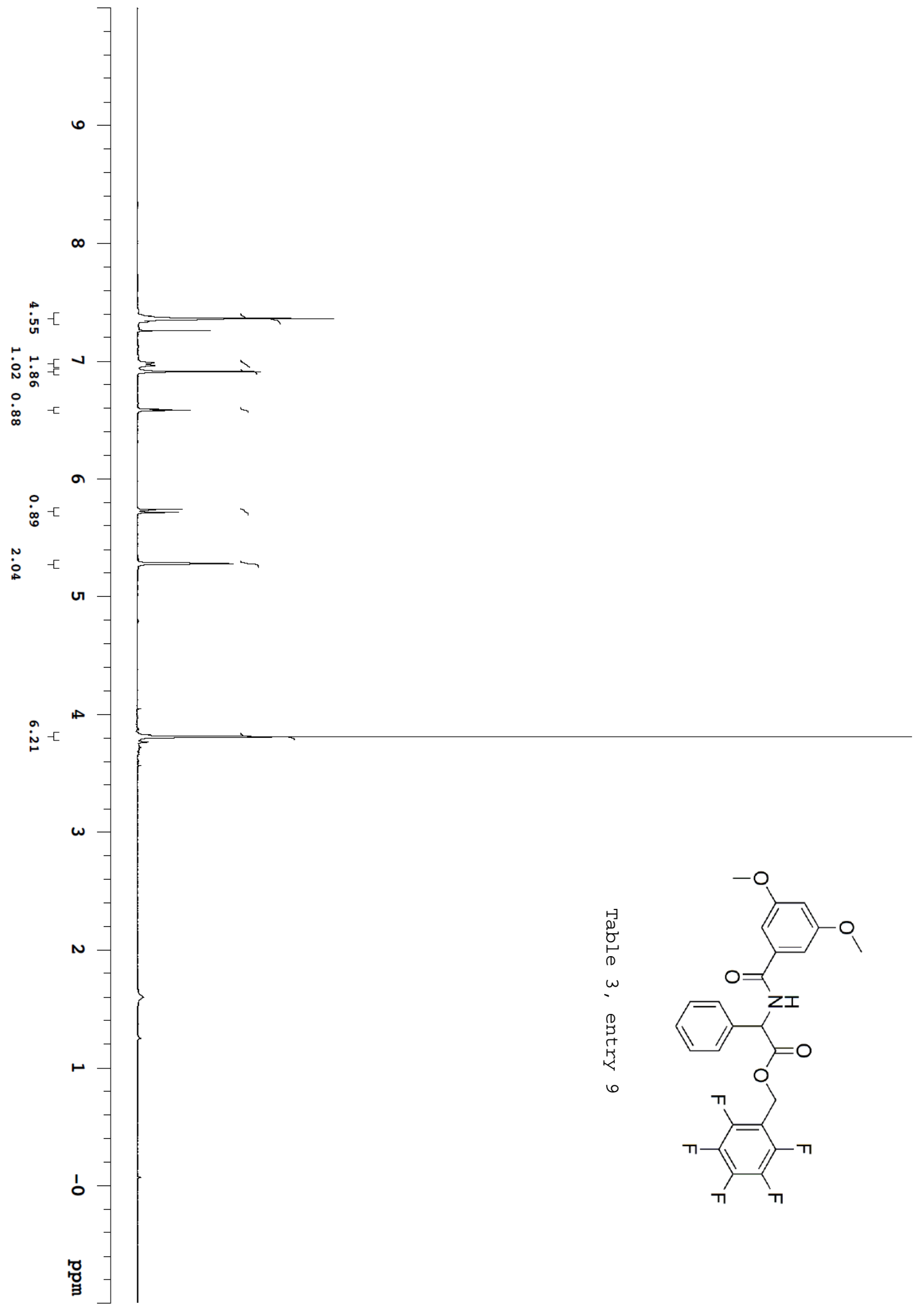



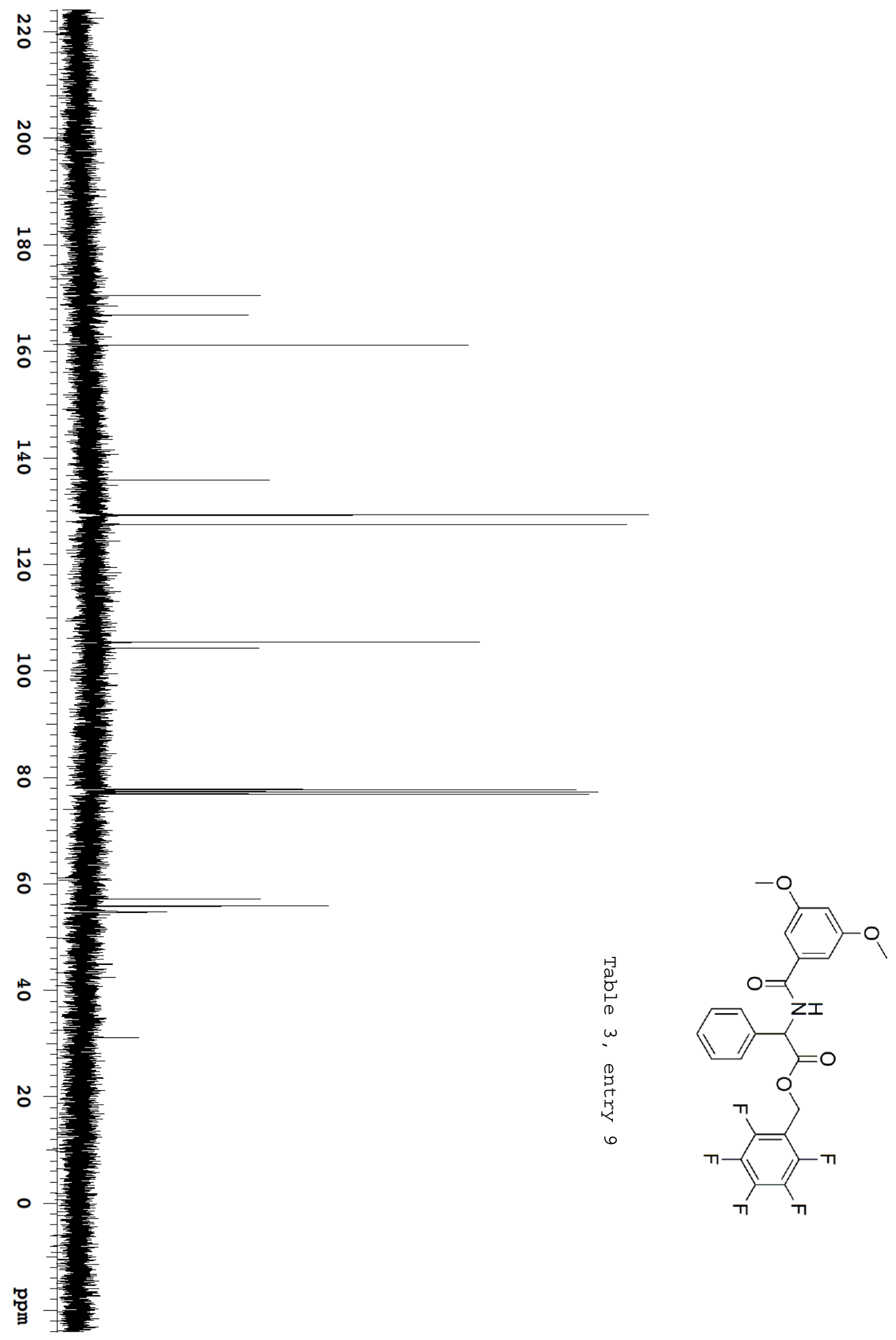


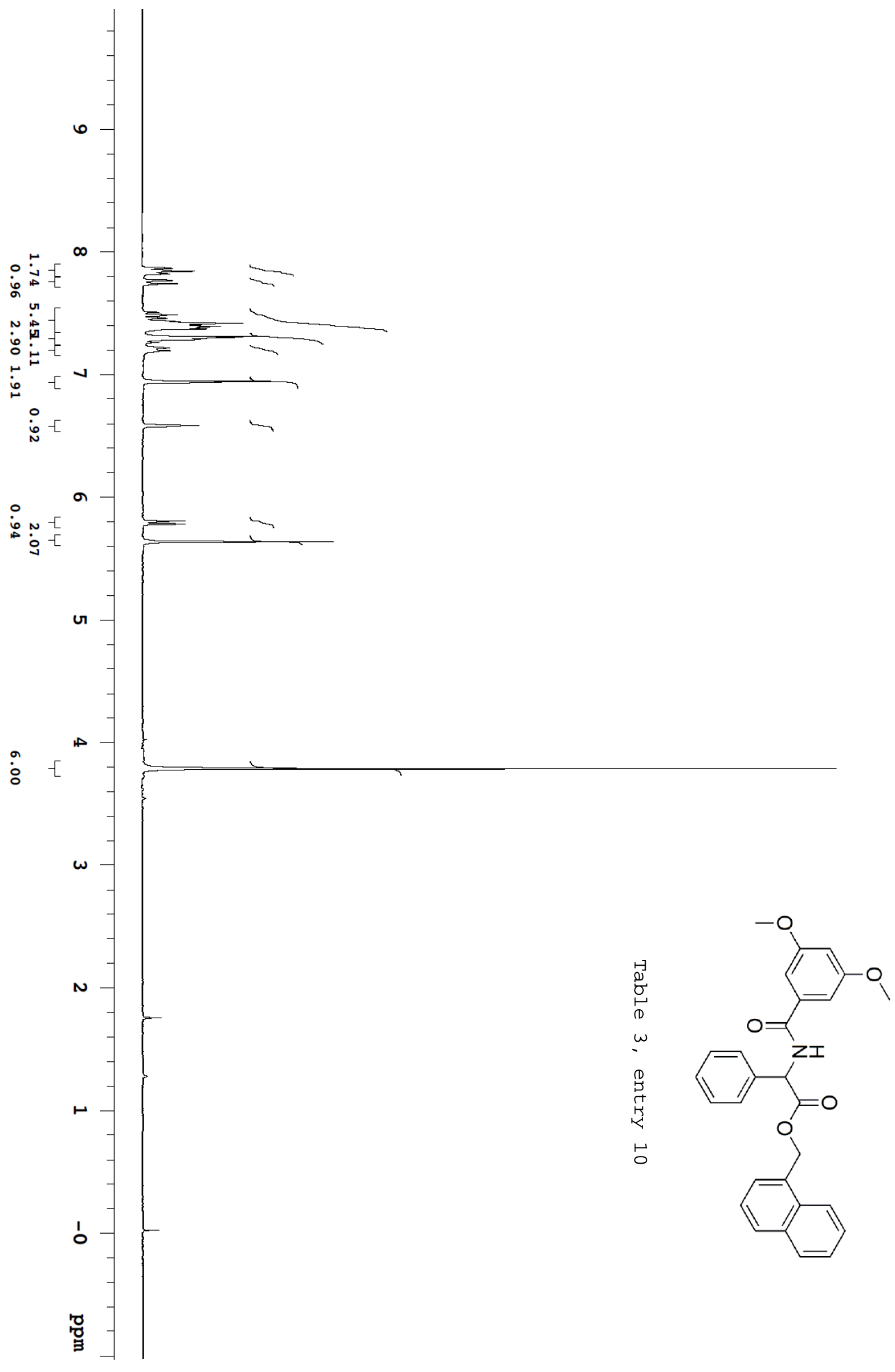



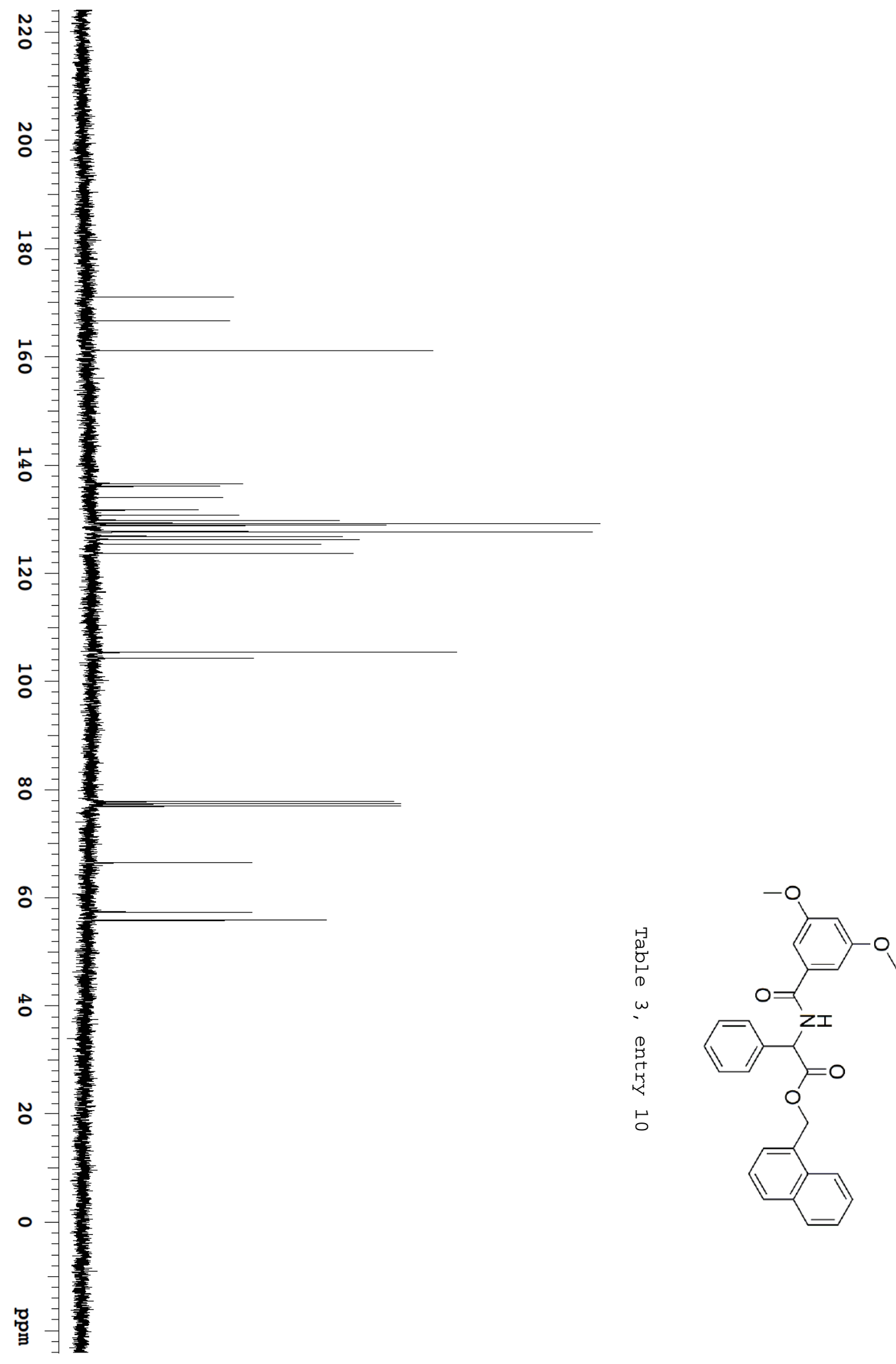


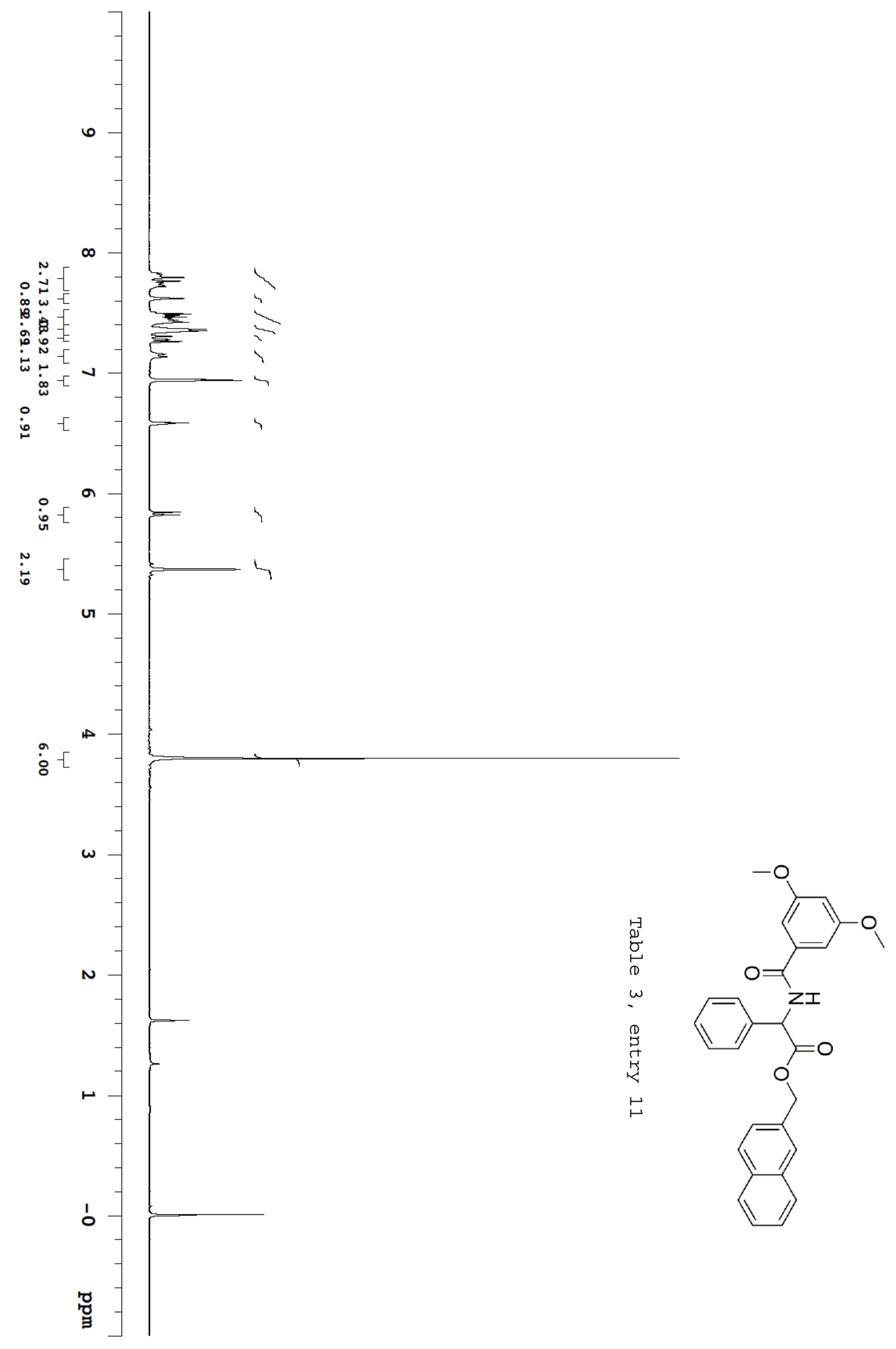




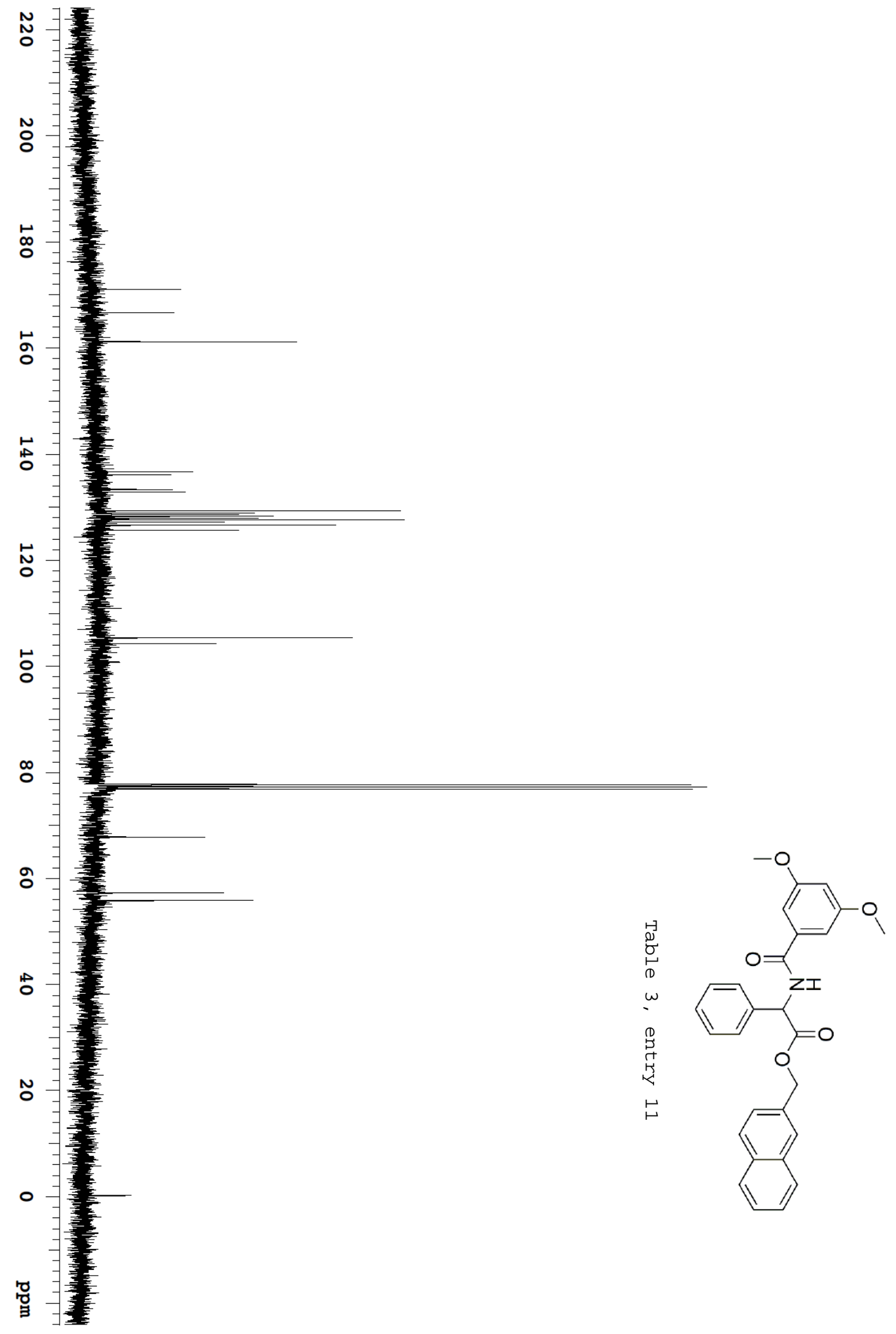




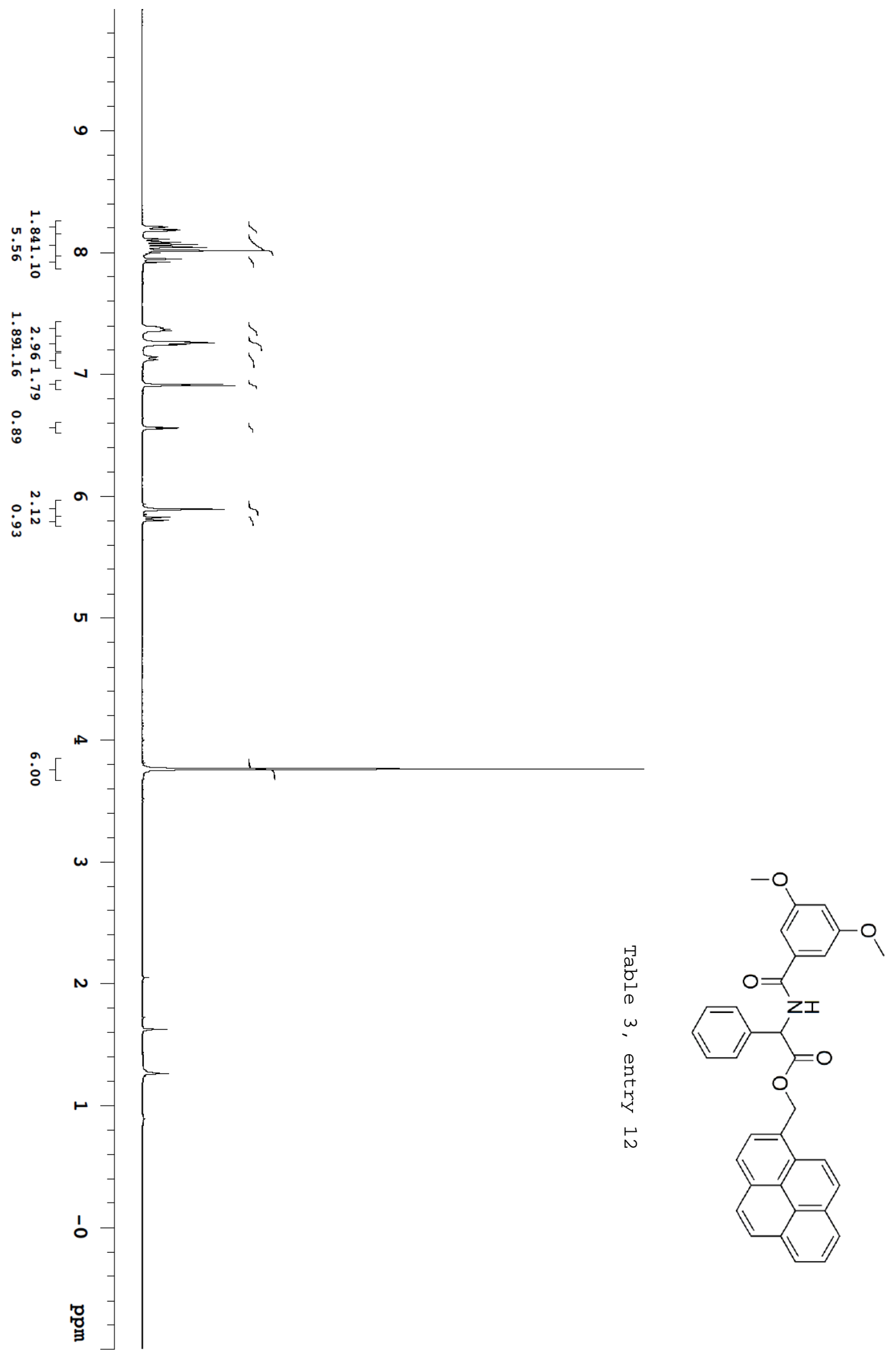



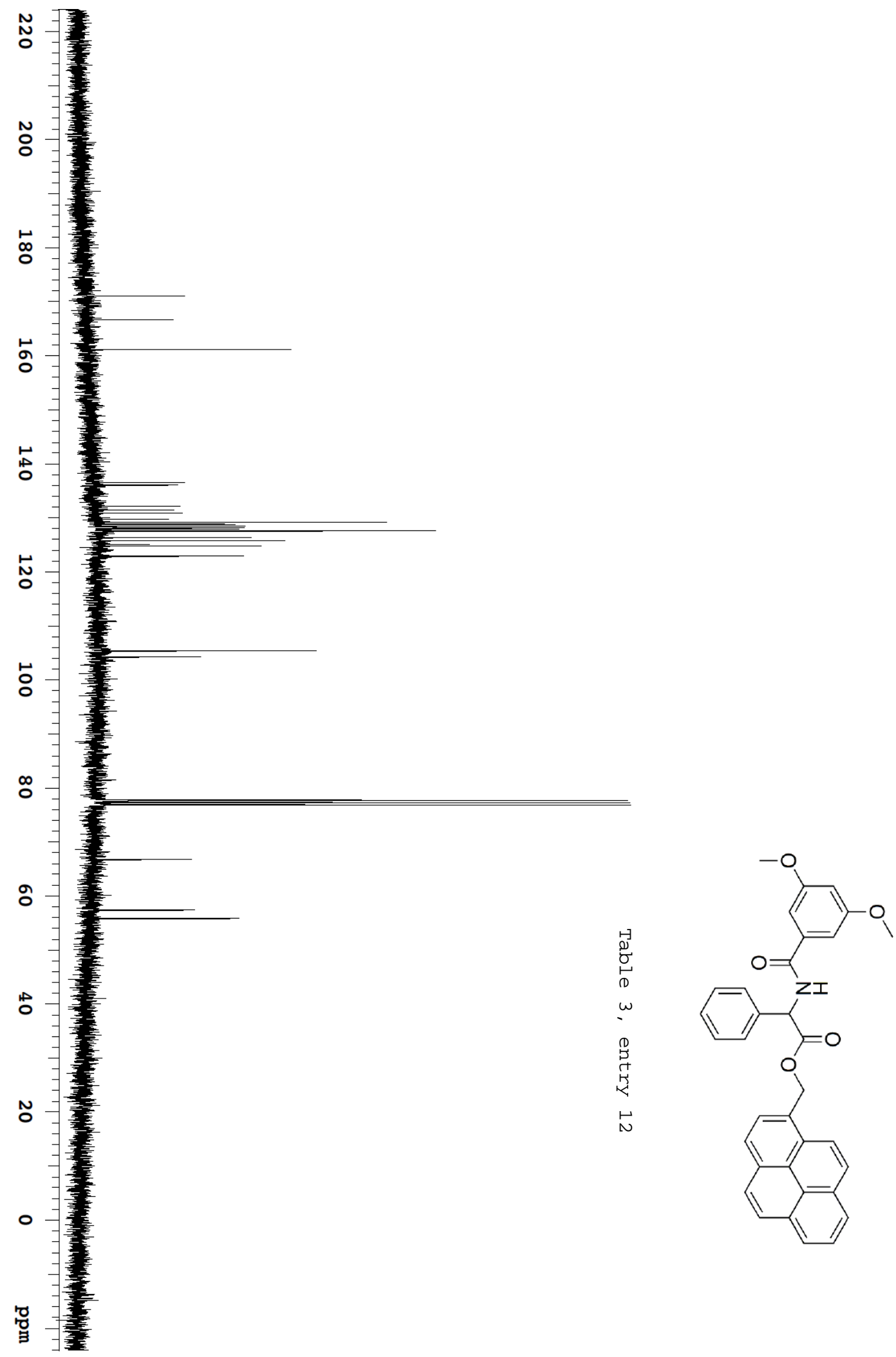


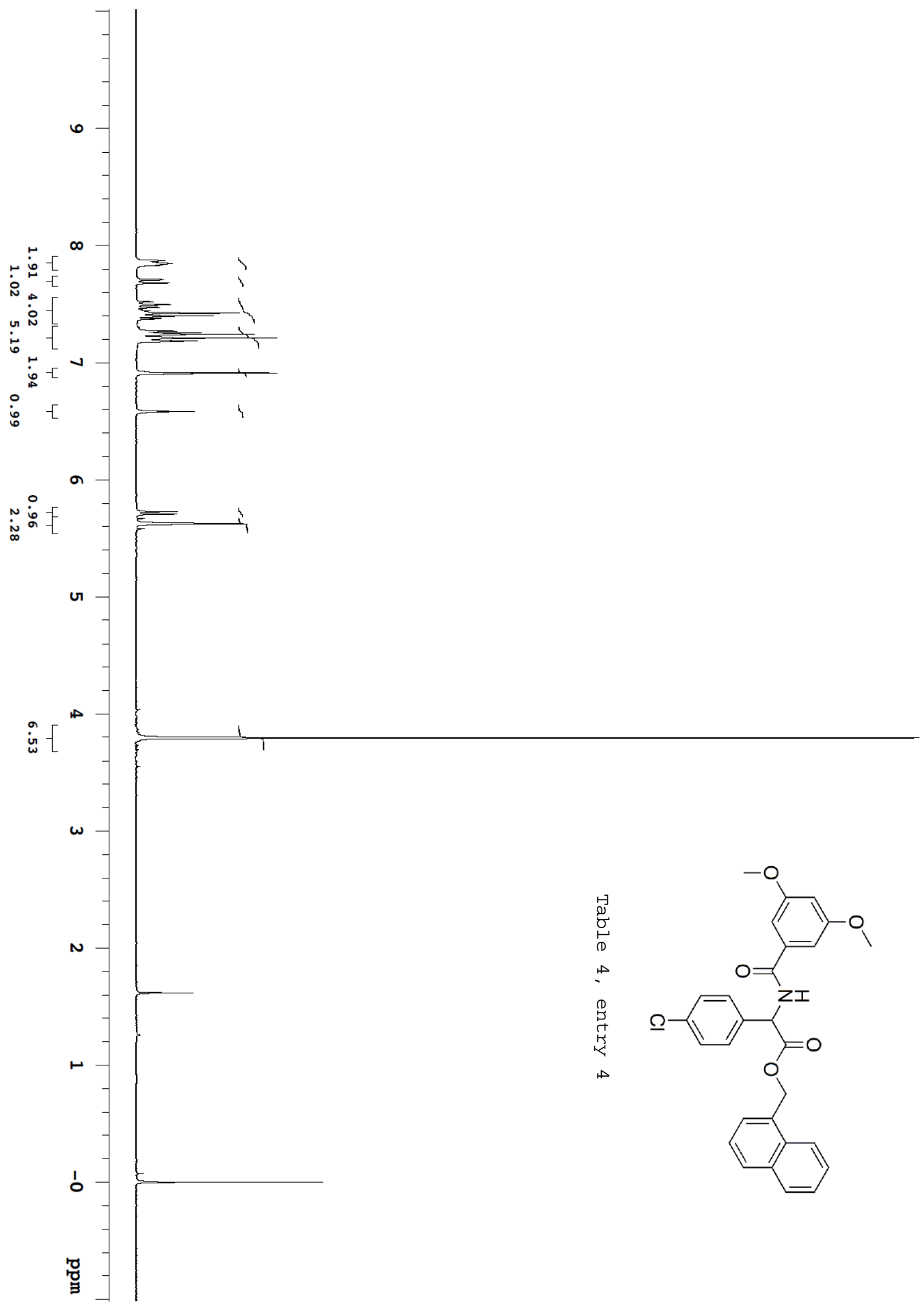




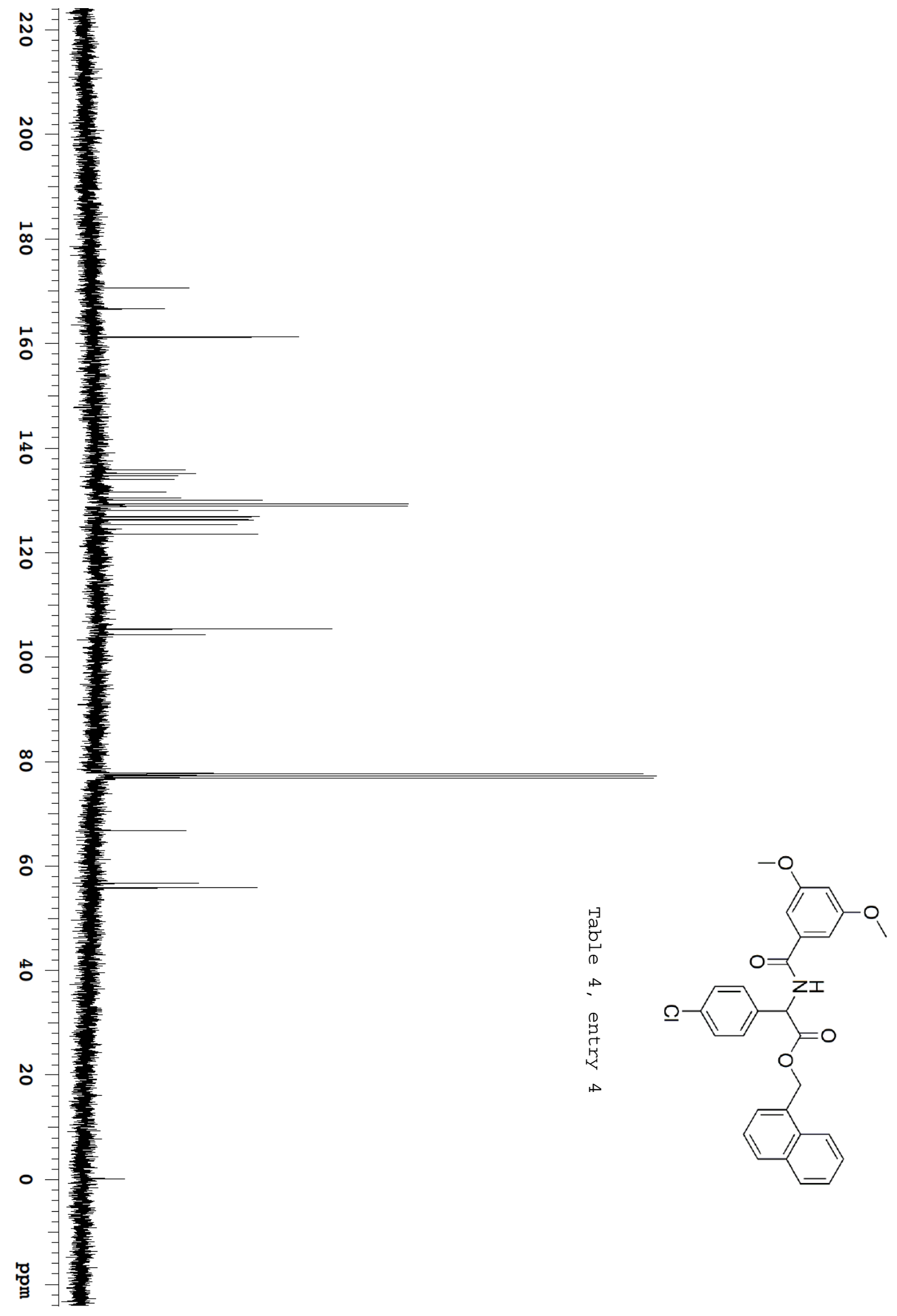




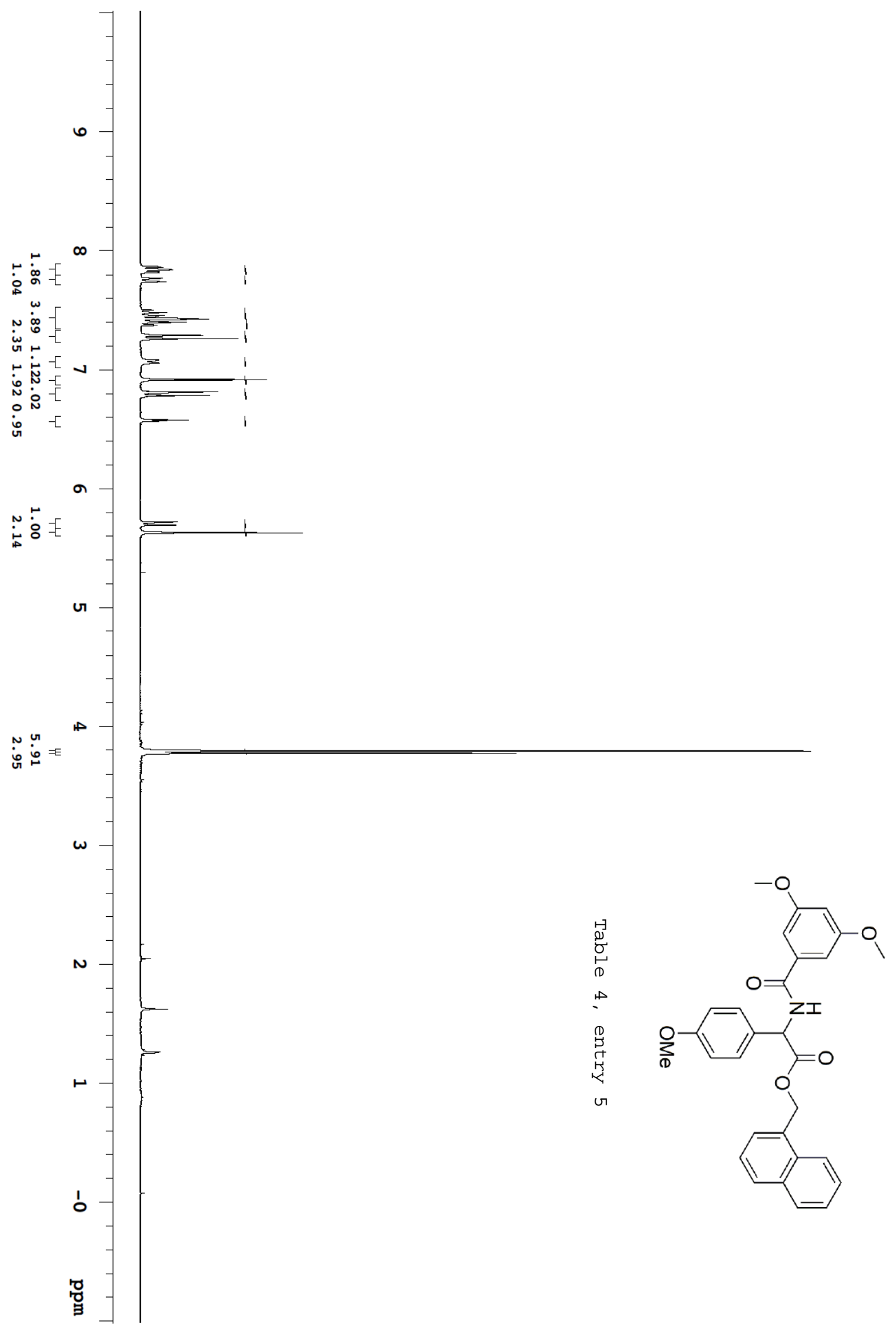




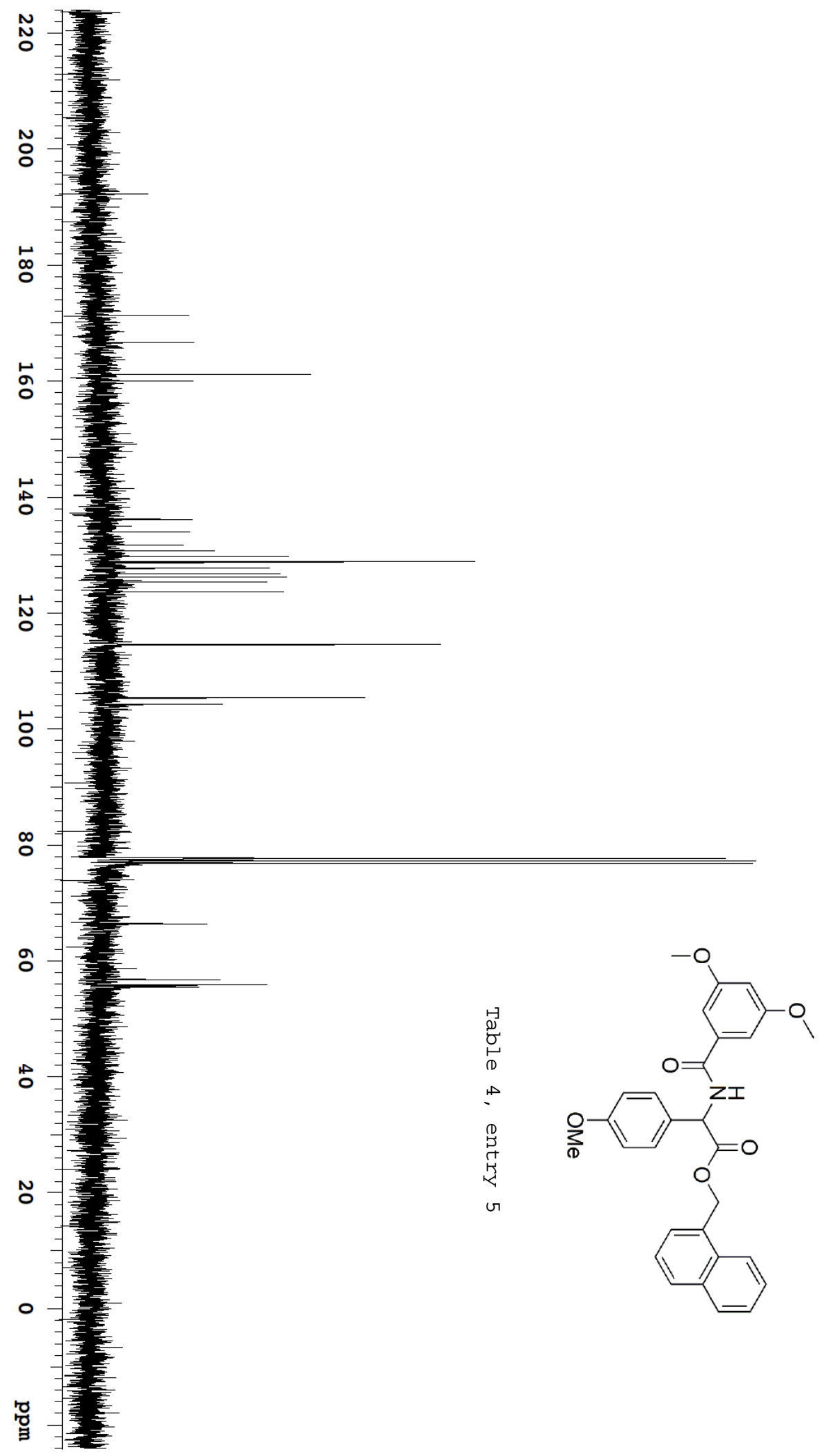




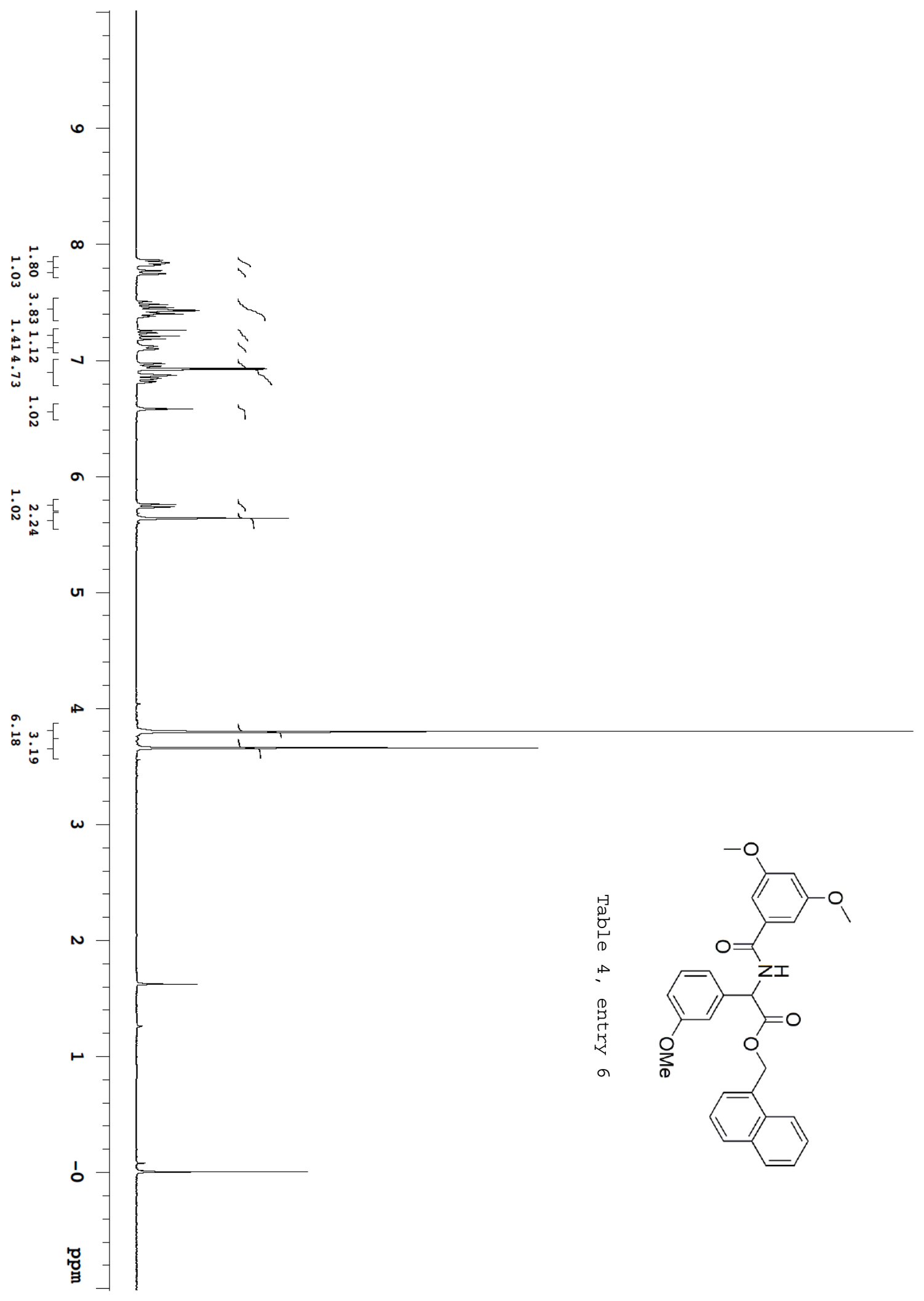




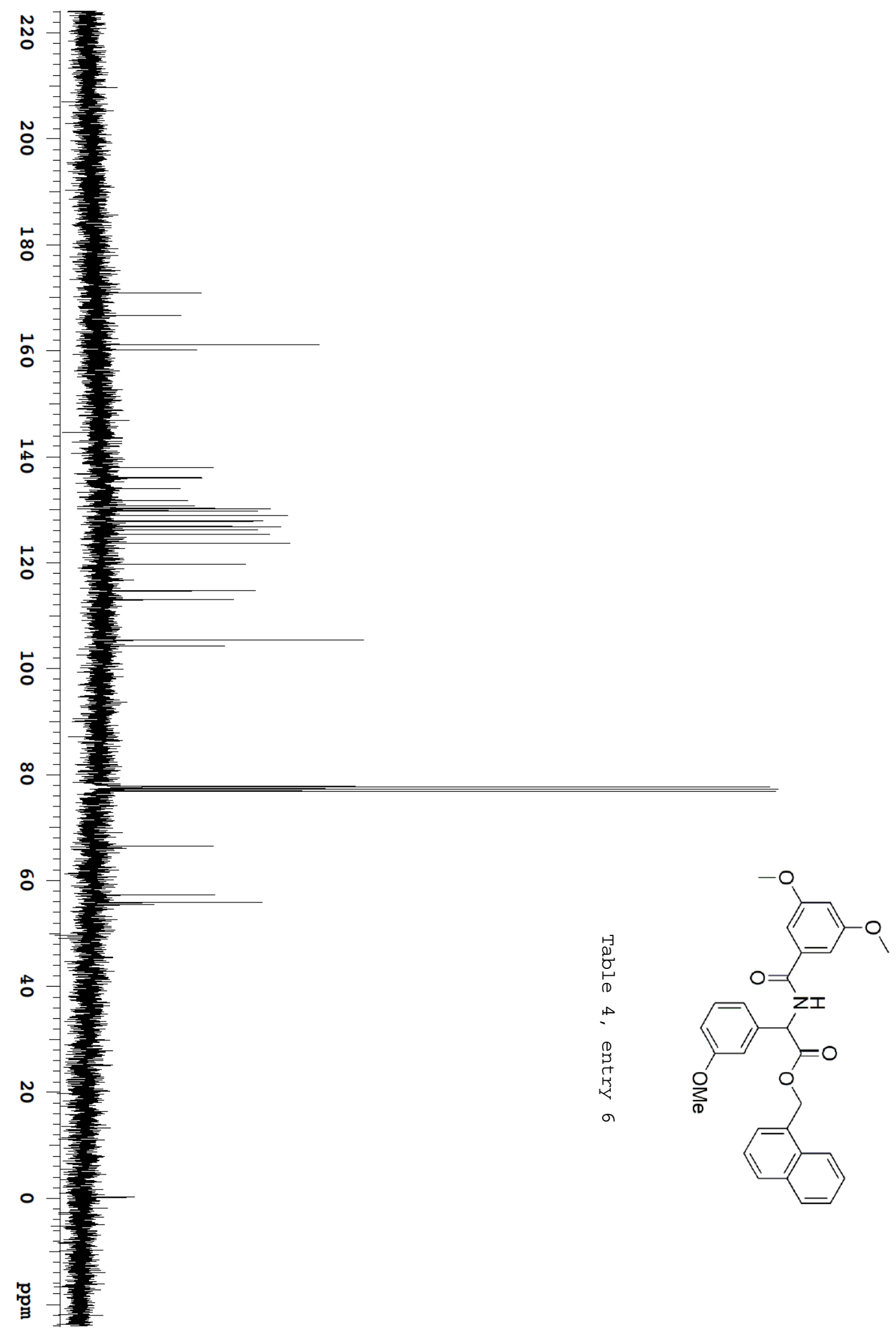




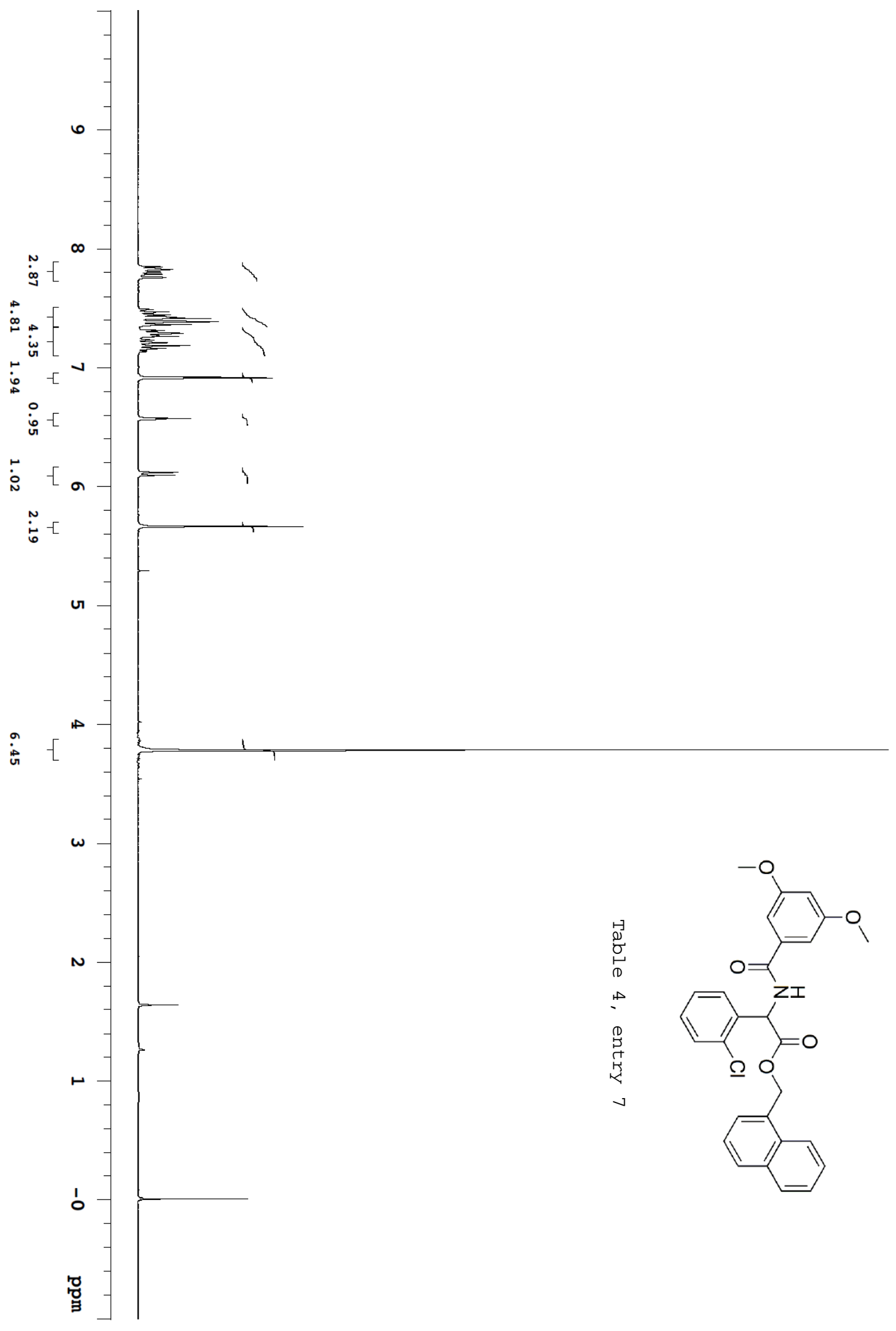




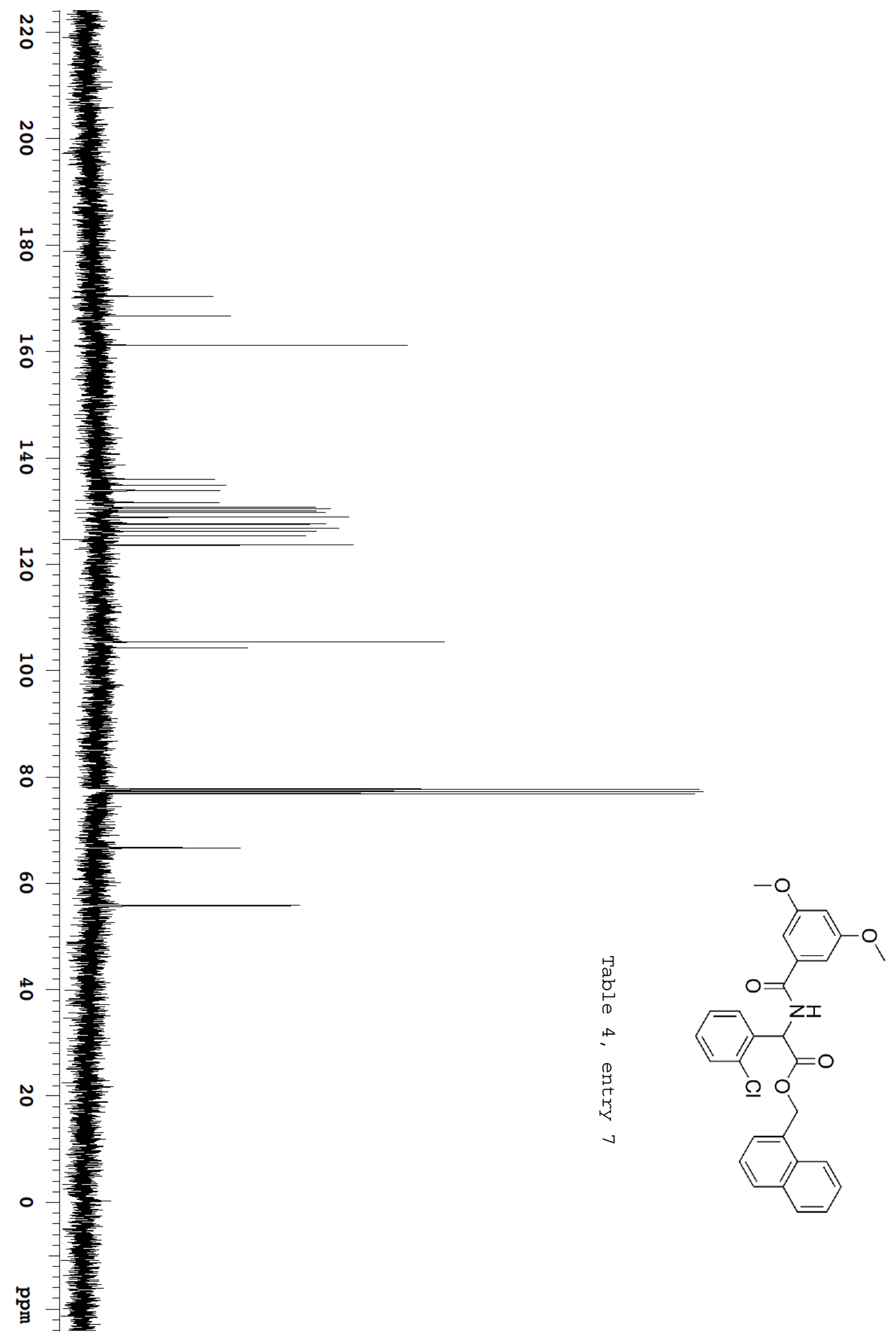




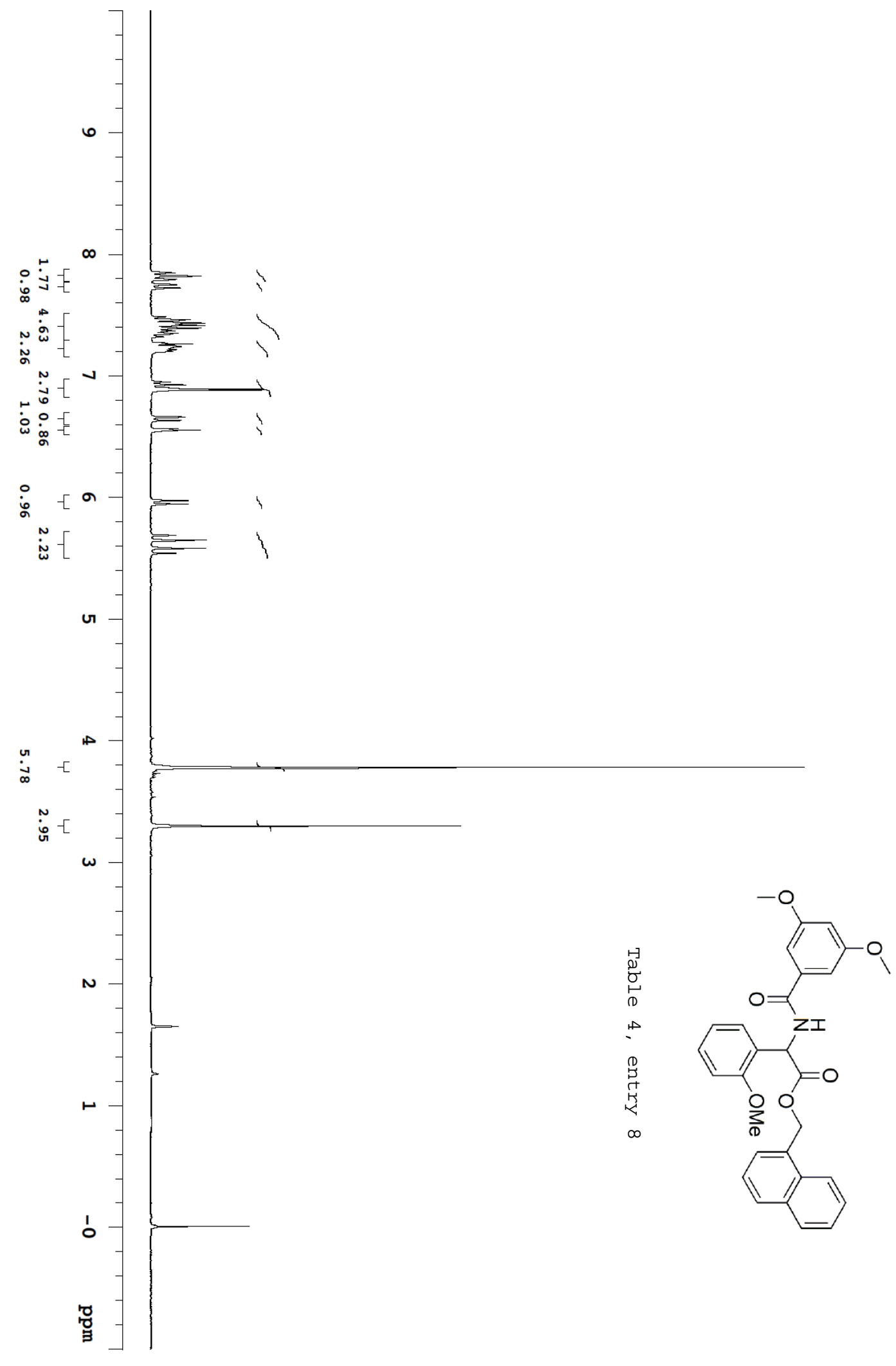



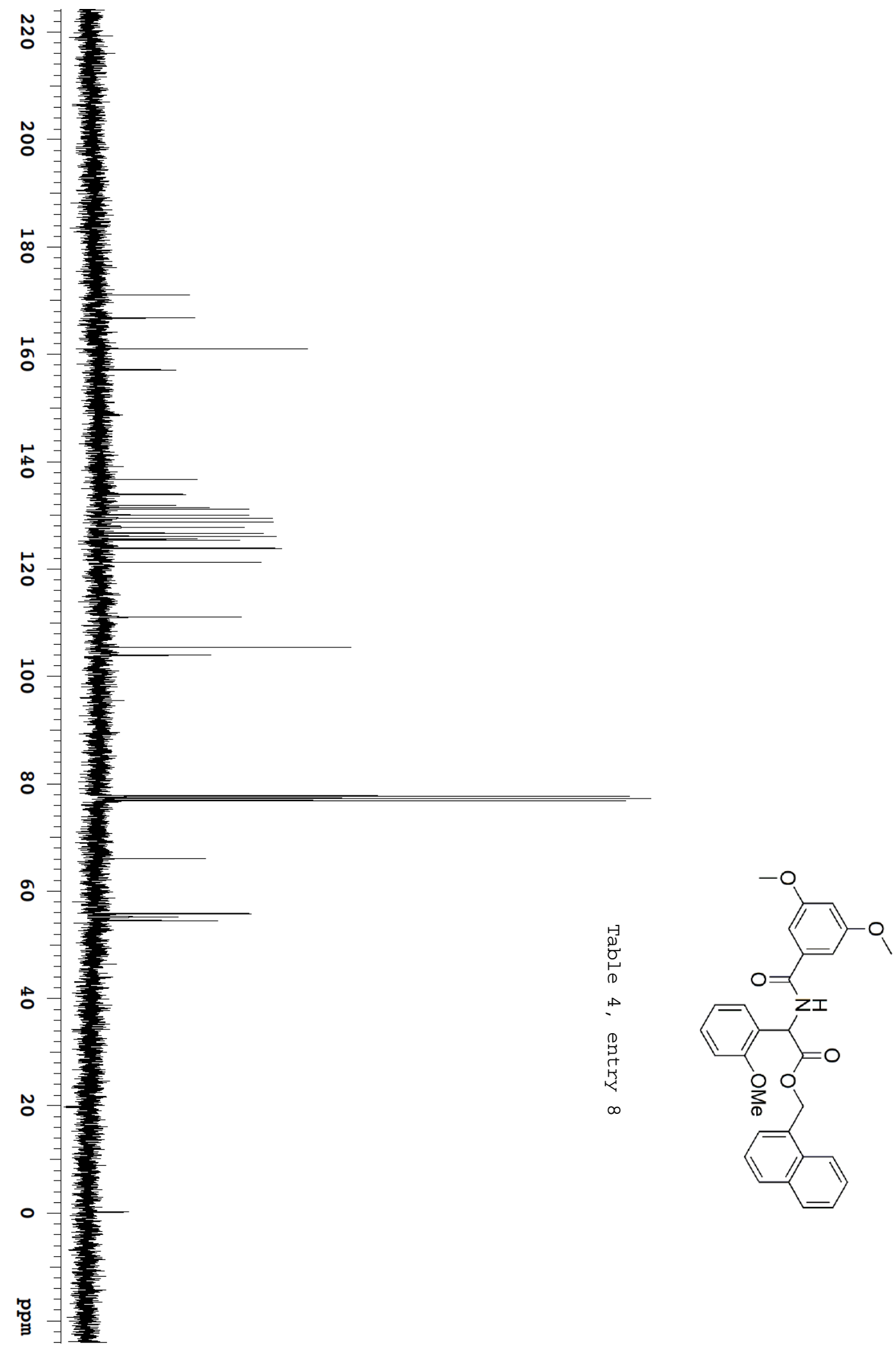


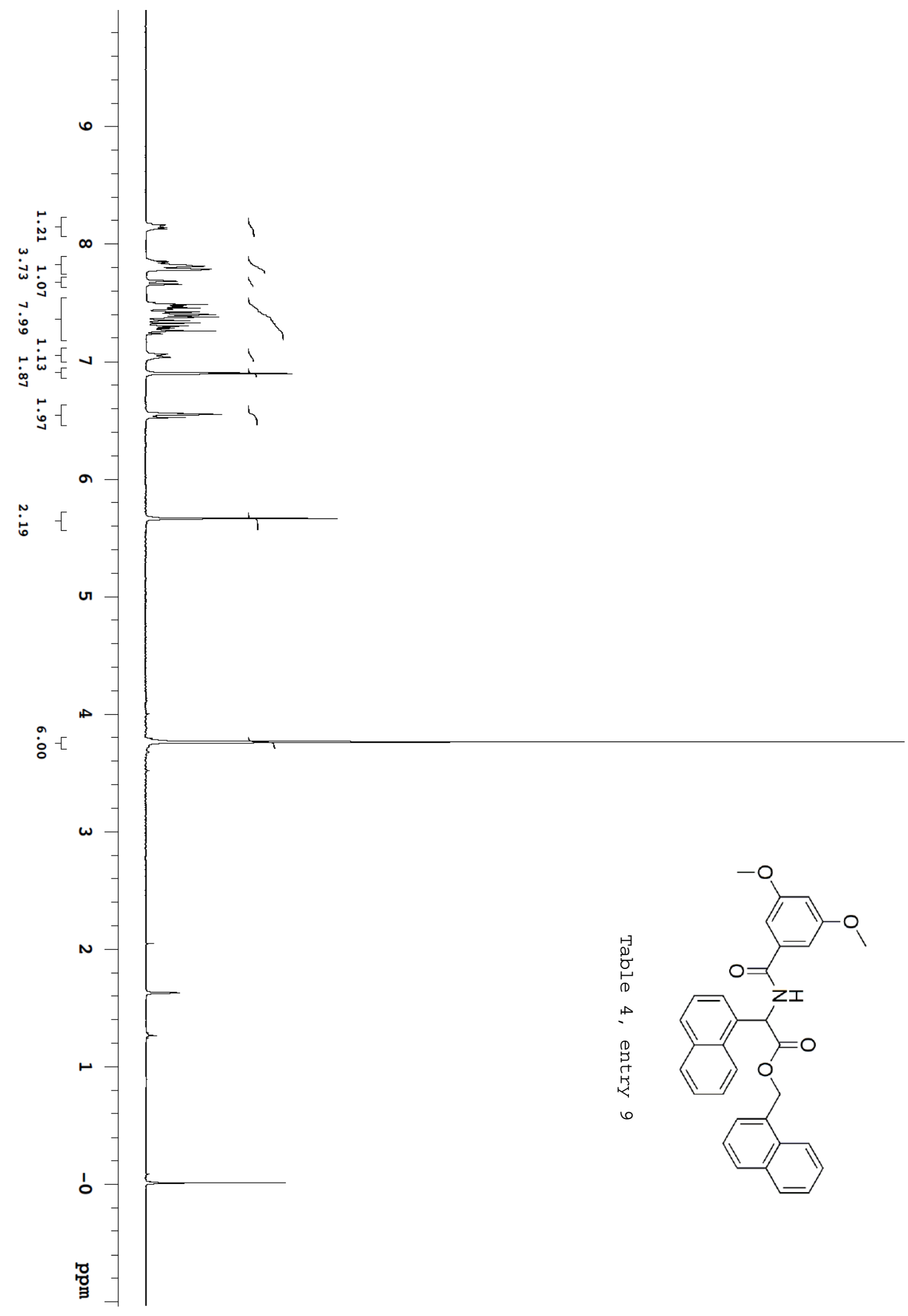




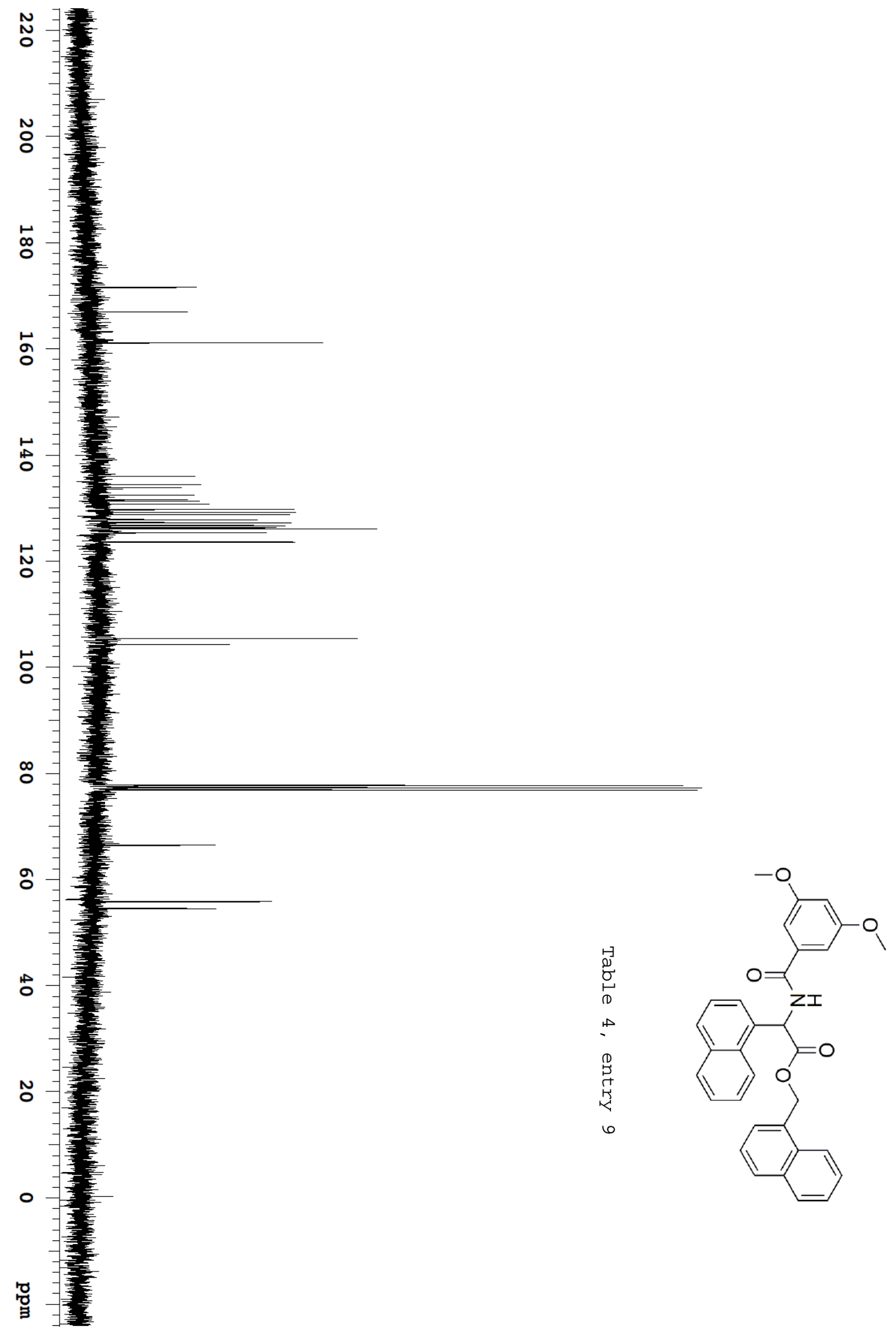




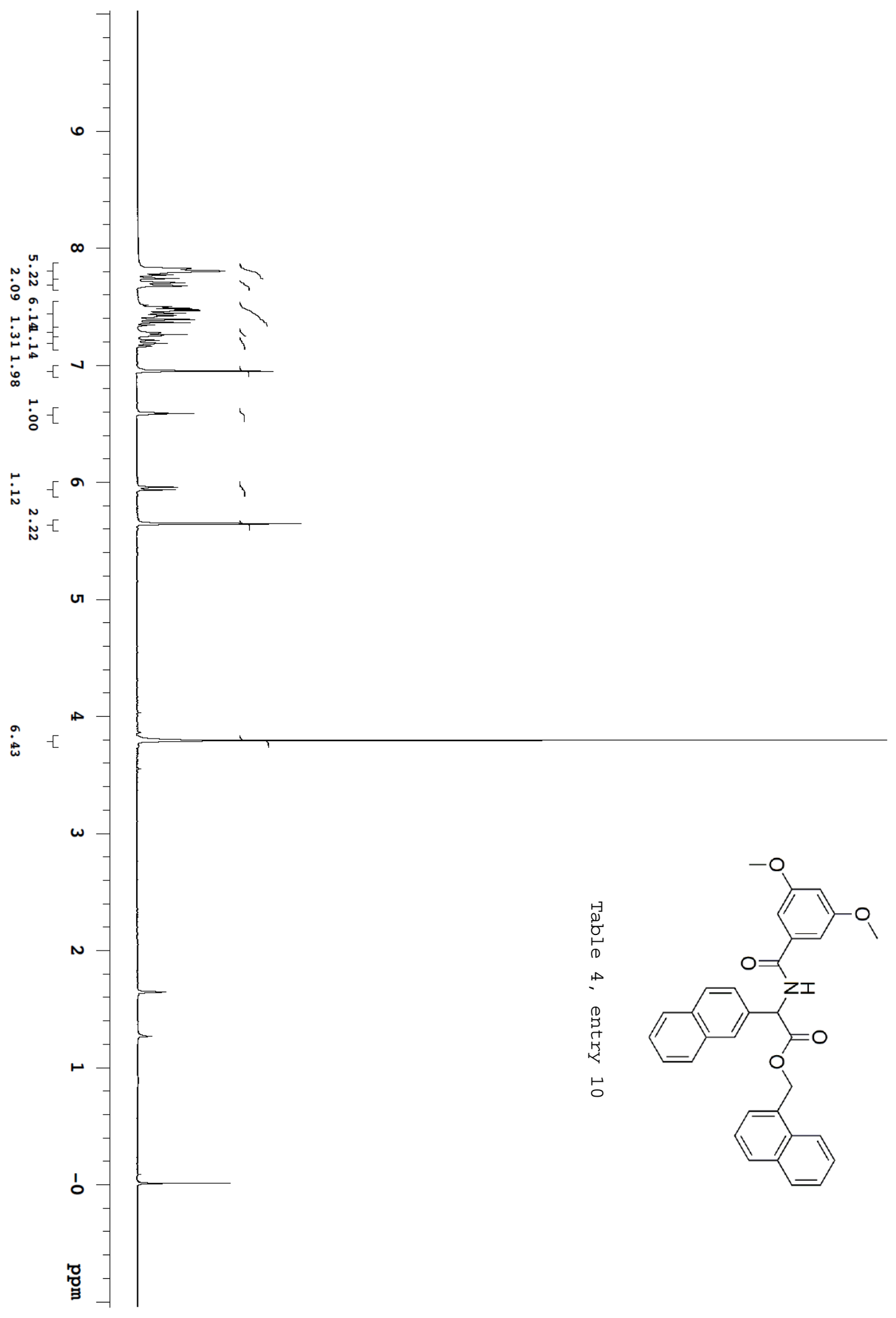




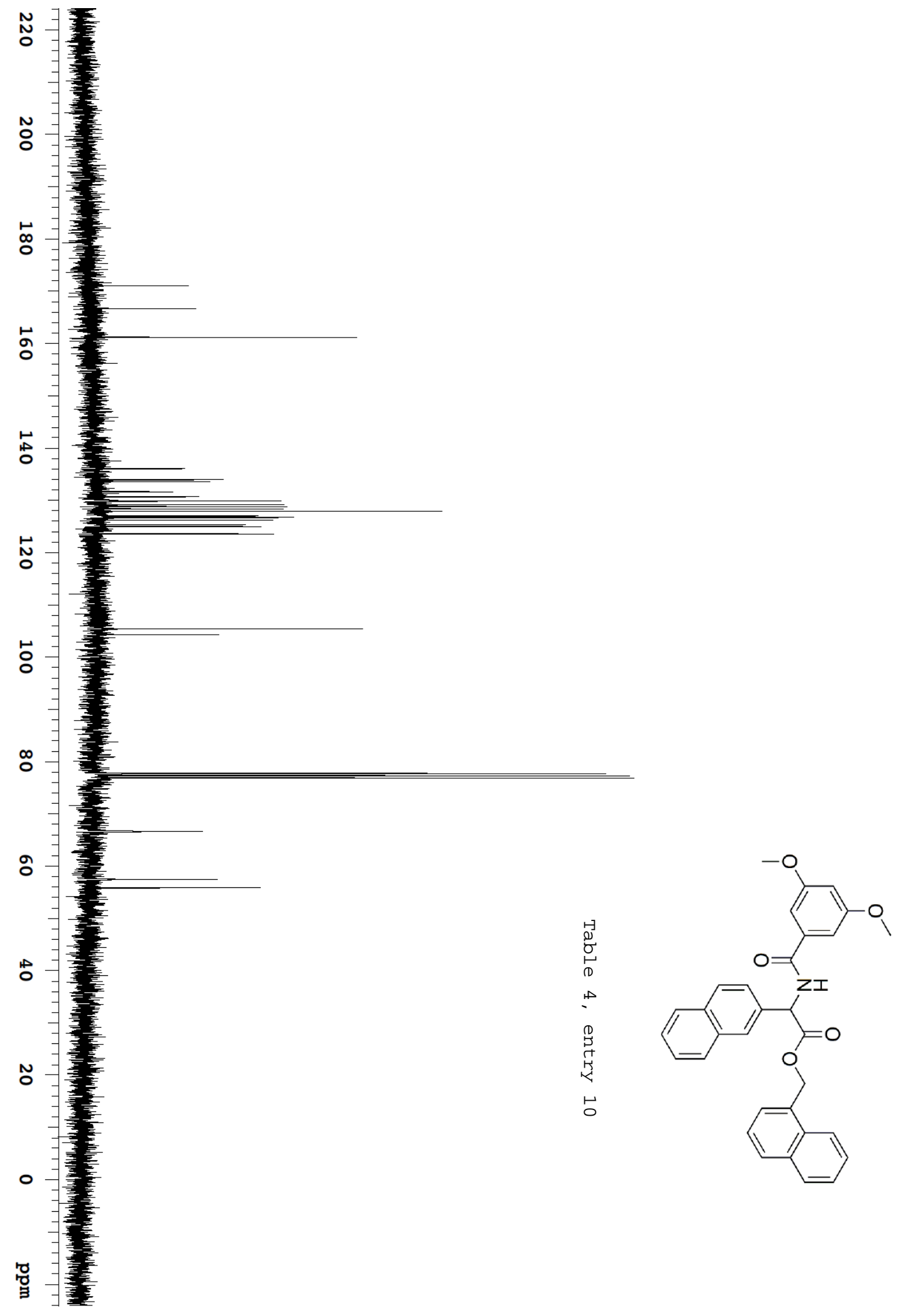




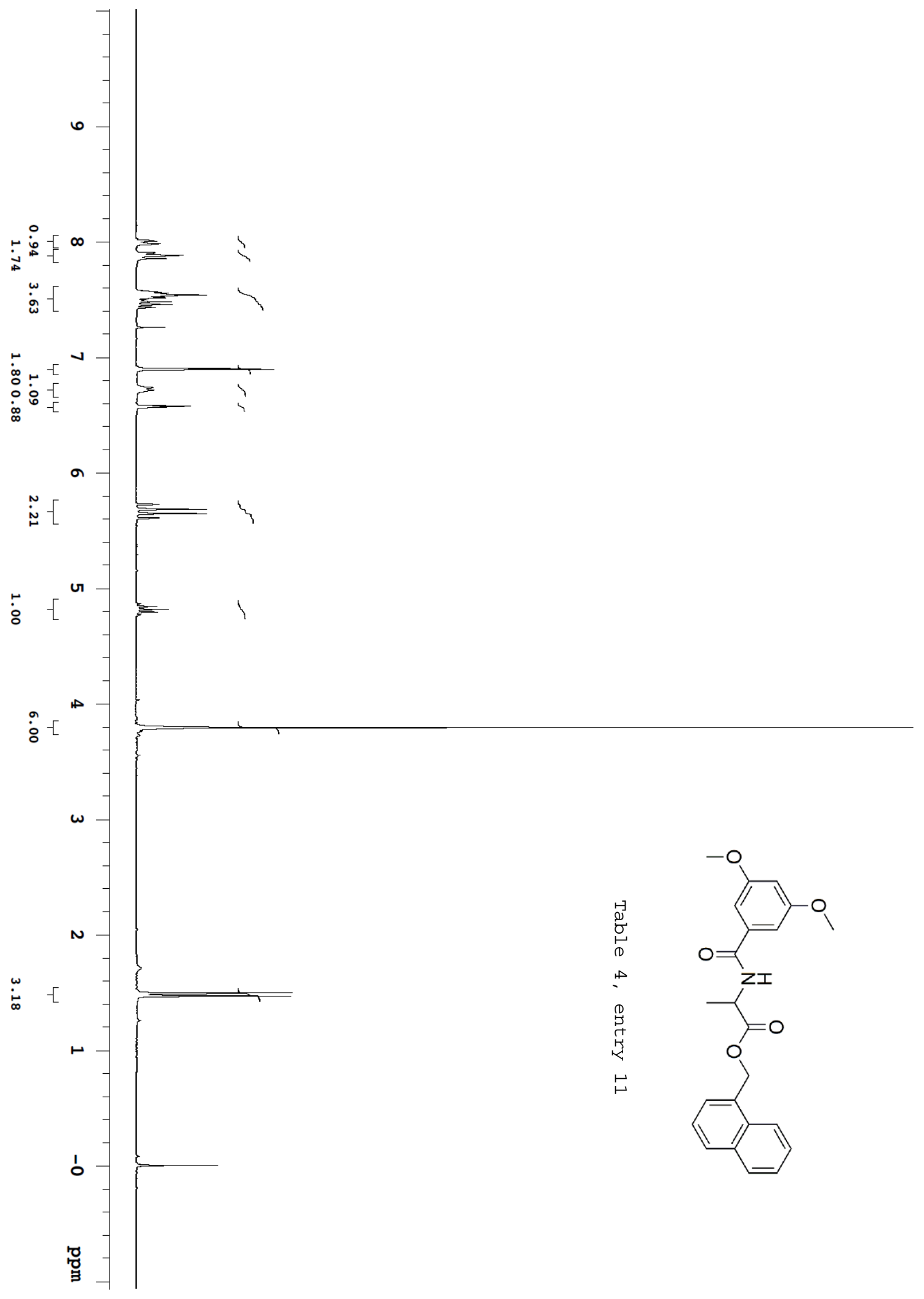



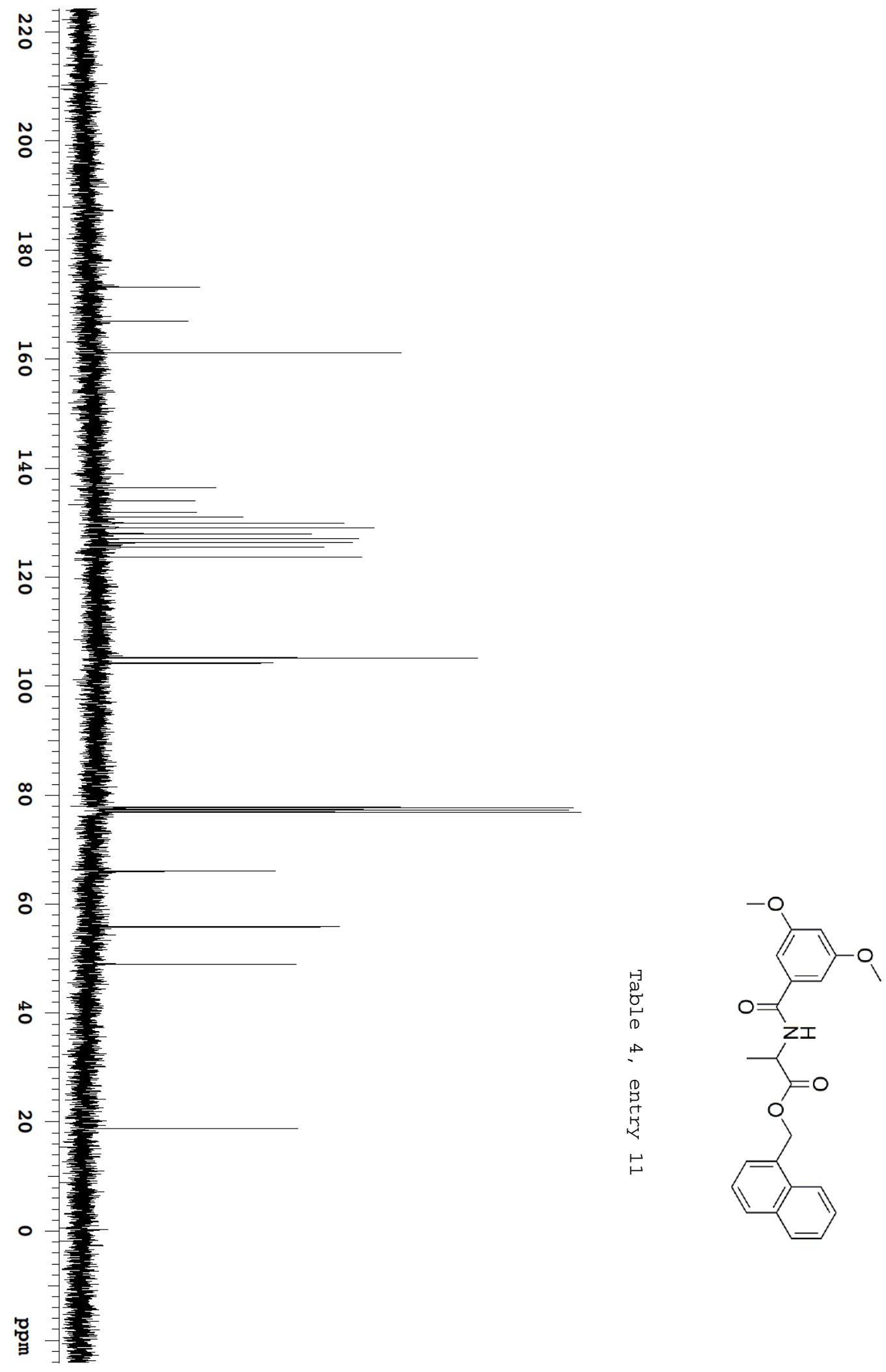


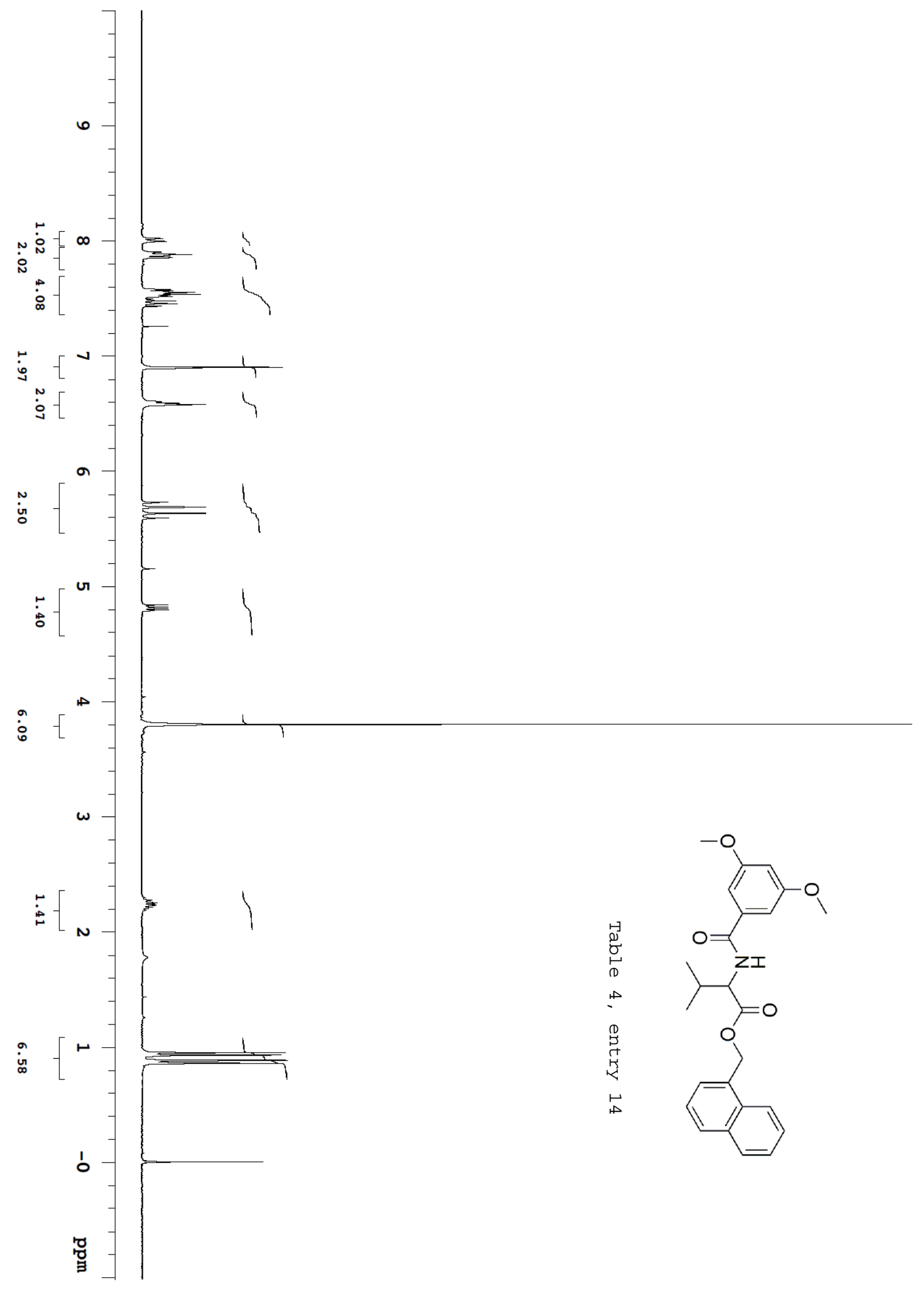




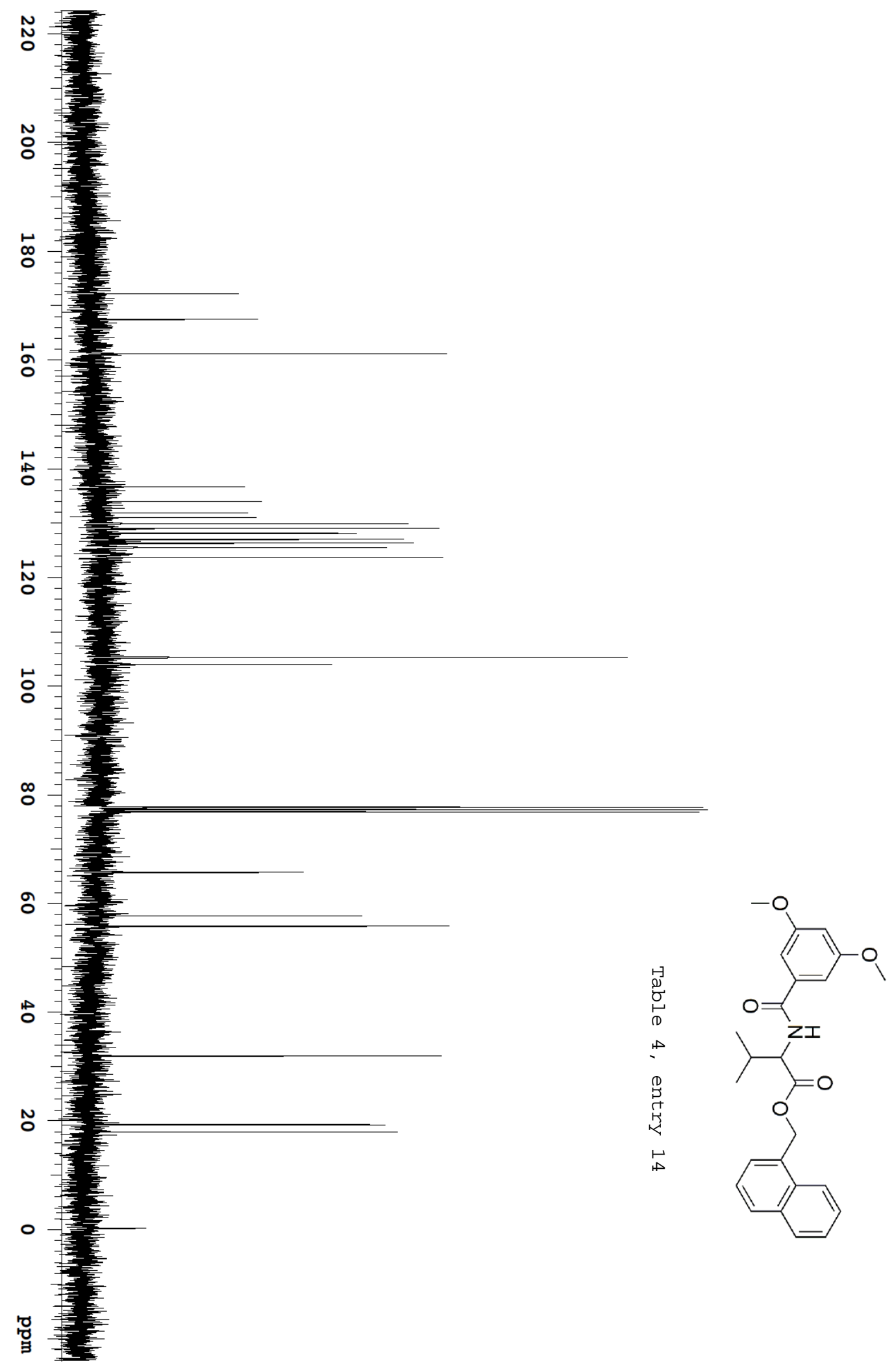

